Portland State University

PDXScholar

Fall 1-23-2020

\title{
Classifying Seabed Parameters from Normal Incidence Reflections: Model Comparison and Inversion Technique
}

Megan Frantz

Portland State University

Follow this and additional works at: https://pdxscholar.library.pdx.edu/open_access_etds

Part of the Electrical and Computer Engineering Commons Let us know how access to this document benefits you.

Recommended Citation

Frantz, Megan, "Classifying Seabed Parameters from Normal Incidence Reflections: Model Comparison and Inversion Technique" (2020). Dissertations and Theses. Paper 5396.

https://doi.org/10.15760/etd.7269

This Thesis is brought to you for free and open access. It has been accepted for inclusion in Dissertations and Theses by an authorized administrator of PDXScholar. Please contact us if we can make this document more accessible: pdxscholar@pdx.edu. 
Classifying Seabed Parameters from Normal Incidence Reflections; Model

Comparison and Inversion Technique

by

Megan Frantz

A thesis submitted in partial fulfillment of the requirements for the degree of

\author{
Master of Science \\ in \\ Electrical and Computer Engineering
}

Thesis Committee:

Martin Siderius, Chair

Richard Campbell

Branimir Pejcinovic

Portland State University

2019 


\begin{abstract}
As of the year 2019, only about five percent of the seafloor has been topologically mapped and classified for type (e.g., sand, silt, gravel). To rapidly survey the seabed from surface ships or underwater vehicles, acoustic remote sensing methods are needed. In this thesis, acoustic measurements and inverse modeling is investigated as a way to classify seabed type based on estimating parameters such as density, sound speed, and interface roughness. The method uses normal incident acoustic measurements that can be made using either a single beam echo sounder or the normal incident beams from a side scan sonar. The inverse method consists of a forward model to simulate the signal and a directed search over parameter space based on an evolutionary algorithm. To direct the search, the similarity between modeled and data envelopes are quantified using a Huber Loss objective function. The large search space requires rapid forward model calculations so a ray-based model was implemented. To determine the applicability of the ray-based model at low frequencies, a validation study is presented to compare the approximate ray method against exact solutions. The full inversion problem was also considered for sediment types of sand, silt and gravel at various signal to noise ratios and those results are included.
\end{abstract}




\section{Contents}

$\begin{array}{ll}\text { Abstract } & \text { i }\end{array}$

List of Figures $\quad$ v

List of Tables viii

\begin{tabular}{lll}
\hline & Introduction & 1
\end{tabular}

2 Parameter Estimation Using Inverse Modeling 5

2.1 Forward Modeling . . . . . . . . . . . . . . . . . . . . . . . 6

2.2 Inversion Methods . . . . . . . . . . . . . . . . . . . 7

3 Background on Propagation and Scattering 10

$3.1 \quad$ Wave Theory $\ldots \ldots$. . . . . . . . . . . . . . . . . . . . . . 10

3.1 .1 Solutions to the Wave Equation . . . . . . . . . . . . 11

3.1 .2 Plane Wave Approximation . . . . . . . . . . . . . . 13

3.2 Transmission Loss . . . . . . . . . . . . . . . . . . . . . . . . . 17

$3.2 .1 \quad$ Geometric Spreading Loss . . . . . . . . . . . . . . . 18

3.2 .2 Attenuation . . . . . . . . . . . . . . . . . 20

$3.3 \quad$ Scattering and Reflection . . . . . . . . . . . . . . . . . . 20

4 Scattering Modeling and Methodology 23

4.1 Motivation . . . . . . . . . . . . . . . . . . . . . . 23

4.2 Model Considerations . . . . . . . . . . . . . . . . . . . . . . . . . . . 23 
$4.2 .1 \quad$ Systems for Classification . . . . . . . . . . . . . . 23

$4.2 .2 \quad$ Model Approximations . . . . . . . . . . . . . . 27

4.3 Seabed Parameters . . . . . . . . . . . . . . . . . . . . . . . 31

4.4 Seabed Classification . . . . . . . . . . . . . . . . . . . . . 32

5 Random Rough Surfaces 34

$5.1 \quad$ Gaussian Roughness Spectrum . . . . . . . . . . . . . . . . . 34

5.2 von-Karman Roughness Spectrum . . . . . . . . . . . . . 35

$6 \quad$ Numerical Model Theory and Validation 39

6.1 Pinson-Cordioli Model . . . . . . . . . . . . . . . . . . . . . . . 39

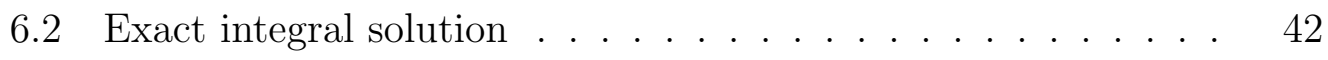

6.3 The Snellen, Siemes, and Simon Model . . . . . . . . . . . 45

6.4 Model Validation . . . . . . . . . . . . . . . . . . 47

$6.4 .1 \quad 10 \mathrm{kHz}$ Source $\ldots \ldots \ldots \ldots$

$6.4 .2 \quad 5 \mathrm{kHz}$ Source $\ldots \ldots \ldots$

$6.4 .3 \quad 1.5 \mathrm{kHz}$ Source $\ldots \ldots \ldots \ldots$

$6.5 \quad$ Summary and Discussion of Modeling Results . . . . . . . 56

\begin{tabular}{lll}
\hline 7 & Inversion and Classification & 58
\end{tabular}

$7.1 \quad$ Objective Function: Huber Loss $\ldots \ldots \ldots \ldots$

7.2 Inverse Methods . . . . . . . . . . . . . . . . . . . 60

7.3 Differential Evolution . . . . . . . . . . . . . . . . 61

7.3 .1 Motivation . . . . . . . . . . . . . 62

7.3 .2 Algorithm . . . . . . . . . . . . . . 63 
7.4 Inversion Structure $\ldots \ldots \ldots$

7.5 Inversion Results $\ldots \ldots \ldots \ldots$. . . . . . . . . . . 67

7.5 .1 Sand . . . . . . . . . . . . . . . . 67

7.5 .2 Gravel . . . . . . . . . . . . . . . . . . . 72

$7.5 .3 \quad$ Silt $\ldots \ldots \ldots \ldots \ldots \ldots \ldots$

7.6 Summary and Discussion of Results . . . . . . . . . . . 82

$\begin{array}{lll}8 & \text { Conclusions and Future Work } & 87\end{array}$

\begin{tabular}{ll}
\hline Bibliography & 93
\end{tabular} 


\section{List of Figures}

2.1 Types of forward modeling . . . . . . . . . . . . . . . . . 7

2.2 Inverse modeling cycle . . . . . . . . . . . . . . . . . . . . . . 9

$3.1 \quad$ Summary of relationships among theoretical approaches for propagation modeling $($ Source $((1))] \ldots$. . . . . . . . . . . . . 14

3.2 Spherical point source . . . . . . . . . . . . . . . . 15

$3.3 \quad$ Spherical wave propagation away from point source . . . . . . 15

$3.4 \quad$ Spherical wave propagation far from point source . . . . . . . 16

3.5 Single point source transmission loss . . . . . . . . . . . . . . 18

$3.6 \quad$ Spherical spreading from point source . . . . . . . . . . . . . . 19

3.7 High level schematic to describe acoustic rough interface scattering (adapted from $(2)) \ldots \ldots \ldots . . \ldots \ldots 21$

$4.1 \quad$ Single beam echosounder (Source (3) $)$. . . . . . . . . . . . . . 25

4.2 Side scan sonar (Source $(3))$. . . . . . . . . . . . . . . . . . 26

5.1 Gaussian random surface . . . . . . . . . . . . . . . . . 35

5.2 von Karman spectrum . . . . . . . . . . . . . . . . . . . . . . 37

5.3 von Karman random surface . . . . . . . . . . . . . . . . . . . 38

$6.1 \quad$ Pinson model layered rough interface geometry (Source (4)) . 42

6.2 Geometry of exact integral equation . . . . . . . . . . . . . . 44

6.3 Geometry of Snellen, Siemes, and Simon Model (Adapted from $(5)$ (5 . . . . . . . . . . . . . . . . 46 
6.4 Pinson model compared to exact integral equation and Snellen envelope model for different smooth surface types at $10 \mathrm{kHz}$. . $\quad 50$

6.5 Pinson model compared to exact integral equation and Snellen envelope model for different rough surface types at $10 \mathrm{kHz}$. . . $\quad 51$

6.6 Pinson model compared to exact integral equation and Snellen envelope model for different smooth surface types at $5 \mathrm{kHz}$. . 52

6.7 Pinson model compared to exact integral equation and Snellen envelope model for different rough surface types at $5 \mathrm{kHz}$. . . 53

6.8 Pinson model compared to exact integral equation and Snellen envelope model for different smooth surface types at $1.5 \mathrm{kHz}$. 54

6.9 Pinson model compared to exact integral equation and Snellen envelope model for different rough surface types at $1.5 \mathrm{kHz}$. . $\quad 55$

$7.1 \quad$ Structure of differential evolution (Source $(6))$. . . . . . . . . 65

7.2 Cost function evaluation for sandy surface parameters at High SNR . . . . . . . . . . . . . . . . . . 68

$\begin{array}{lll}7.3 & \text { Real and estimated signal for sandy surface at high SNR . . . } 69\end{array}$

7.4 Cost function evaluation for sandy surface parameters at $20 \mathrm{~dB}$ SNR . . . . . . . . . . . . . . . 70

7.5 Real and estimated signal for sandy surface at 20dB SNR . . . $\quad 70$

7.6 Cost function evaluation for sandy surface parameters at 10dB snr . . . . . . . . . . . . . . . . . . . 71

$7.7 \quad$ Real and estimated signal for sandy surface at 10dB SNR . . . $\quad 72$ 
7.8 Cost function evaluation for gravel surface parameters at High SNR . . . . . . . . . . . . . . . . 73

7.9 Real and estimated signal for gravel surface at High SNR . . . 74

7.10 Cost function evaluation for gravel surface parameters at 20dB SNR . . . . . . . . . . . . . . . . . . 75

7.11 Real and estimated signal for gravel surface at 20dB SNR . . . 75

7.12 Cost function evaluation for gravel surface parameters at 10dB SNR . . . . . . . . . . . . . . . 76

7.13 Real and estimated signal for gravel surface at 10dB SNR . . . $\quad 77$ 7.14 Cost function evaluation for silt surface parameters at $40 \mathrm{~dB}$ snr 78

7.15 Real and estimated signal for silt surface at 40dB SNR . . . . 79

7.16 Cost function evaluation for silt surface parameters at 10dB snr 80

7.17 Real and estimated signal for silt surface at 20dB SNR . . . . 80

7.18 Cost function evaluation for silt surface parameters at $10 \mathrm{~dB}$ SNR . . . . . . . . . . . . . . . . 81

7.19 Real and estimated signal for silt surface at 10dB SNR . . . . 82 


\section{List of Tables}

4.1 Table of General Sediment Parameter Values (adapted from (4)) 33

6.1 RMS Height and Correlation Length for Simulated Rough Interfaces $\ldots \ldots \ldots \ldots \ldots \ldots \ldots$

$7.1 \quad$ Inversion Results For Sand at High SNR . . . . . . . . . 68

7.2 Inversion Results For Sand at 20dB SNR . . . . . . . . . . 69

7.3 Inversion Results For Sand at 10dB SNR . . . . . . . . . . . 71

7.4 Inversion Results For Gravel at High SNR $\ldots \ldots \ldots \ldots$

7.5 Inversion Results For Gravel at 20dB SNR . . . . . . . . . . 74

7.6 Inversion Results For Gravel at 10dB SNR . . . . . . . . 76

7.7 Inversion Results For Silt at High SNR . . . . . . . . . 78

7.8 Inversion Results For Silt at 20dB SNR . . . . . . . . . . 79

7.9 Inversion Results For Silt at 10dB SNR . . . . . . . . . . . . 81

7.10 Parameter Ranges for Sediment Type (adapted from (4)) . . . 83

7.11 Parameter Ranges for Roughness Type (adapted from (4)) . . 83

7.12 Classification Results: Seabed Type . . . . . . . . . . . . . 84

7.13 Classification Results: Roughness Type . . . . . . . . . . . 85 


\section{Chapter 1}

\section{Introduction}

As of the year of 2019, only about five percent of the seafloor has been topologically mapped and classified. If you consider that seventy percent of the Earth is covered in water, this percentage seems insignificant. There are a variety of reasons to understand the seabed type. For example, for deploying equipment on the seabed it is important to know if the mooring will bury or will be sitting proud. There are similar concerns that arise when laying cables on the seabed. It is also common to use sonars to search over the seabed (e.g., for downed airplanes, unexploded ordinance or ship wrecks) and objects can be fully or partially buried in silts or sands. Searching over rock and other severely rough seabeds usually leads to many artifacts (false detections) in a sonar image. Knowing the seabed type, therefore, leads to better decisions about how (or if) to operate at a particular site.

To efficiently explore and map the seabed, acoustic remote sensing methods have become an important field of study. Acoustic waves propagating in the ocean are subject to a possibly dynamic and rough upper boundary (sea-surface) and a static but rough lower boundary (seabed) (2). Acoustic reflections from the seabed changes the waveform amplitude and phase in a way that depends on the seabed sound speed, density and interface roughness. The remote sensing method considered here is based on inverse modeling. In this 
case, acoustic measurements (data) are compared with a parameterized numerical signal model. The model parameters (such as seabed sound speed, density and roughness) are adjusted and the output is compared against the data and agreement is quantified with an objective function. A search is conducted over a parameter space and the minimum value of the objective function represents the best estimate for the seabed parameters. If the parameter set is small enough, an exhaustive search is made but for larger parameter spaces, this is not feasible and an alternative approach to finding the optimal parameters is needed. Here, the parameter space is large so an evolutionary algorithm is used to greatly reduce the required forward model evaluations.

A major part of the inverse modeling method requires an accurate forward model that captures the acoustic propagation and scattering and can represent a realistic, received signal (7). However, somewhat in opposition to this is the simultaneous need for a forward model to be computationally efficient since typically many evaluations are needed. These constraints can make model selection tricky. There are a fairly large number of forward models to choose from in underwater acoustics depending on the application. Some are more phenomenological (empirical or measurement-based) while a large number of the more accurate ones are based on the physics of wave theory (1). Of the physics based models, the choice generally comes down to the frequency regime and the trade-offs between accuracy and speed (based on various approximations) (8). To balance efficiency and the required accuracy, a ray-based approach was selected here. A new ray model that can treat rough interfaces has recently 
been developed (4) and an extensive validation study of this model was conducted as part of this thesis. This was necessary to confirm the validity of the ray model in the frequency regime being considered here. The ray model was compared against an exact solution based on the Helmholtz-Kirchhoff integral equation.

After a forward model has been selected, the search algorithm and cost function are chosen to solve the specific inverse problem. The cost function used here, the Huber loss, compares the envelopes of the model with the received echo from either a single beam echo sounder or from the normal incident beam on a side scan sonar. The seabed type modifies the envelope shape so the cost function has to be sensitive to the various parameters being inverted for. Huber loss is a combination of mean absolute error and mean squared error. It has a tunable parameter that determines how to balance error functions at a specific point. The ability to switch between these two error functions makes it robust to noise and outliers.

To avoid an exhaustive parameter search, a evolutionary algorithm is used. While similar approaches have been considered for other underwater acoustic applications, this is the first time evolutionary algorithms have been used for single beam seabed classification. Evolutionary algorithms describe a subset of dynamic programming inspired by biological structures that minimize a cost function. There are a wide variety of evolutionary algorithms to chose from when performing parameter estimation including particle swarm optimization, bees algorithm and ant colony optimization. Differential evolution was selected 
as the optimization strategy in this research.

The main objective of this thesis is to develop a seabed classification method based on measurements of normal incident acoustic reflections. The method developed uses inverse modeling with parameter estimation based on an evolutionary algorithm. For this, an efficient yet accurate forward model was selected and thoroughly tested against multiple physics-based scattering models using a variety of rough interfaces. For the first time for this application, the Huber loss cost function was implemented with a differential evolution algorithm. In the final chapter of this thesis, the complete inversion process is tested using simulations for different seabed types and roughness as well as for a range of signal to noise ratios. 


\section{Chapter 2}

\section{Parameter Estimation Using Inverse Modeling}

The inverse problem that is being investigated here is for seabed parameter estimation. Parameter estimation problems can be solved with multiple search strategies and objective or cost functions that determine the best fit between simulated (modelled) acoustic fields and measurement data from sonar sensors (7). The inversion method and cost function are dependent upon the precision required in the seabed parameters (9). In order to select the proper forward model, this chapter will review parameter estimation and inversion modeling.

Inverse problems, in ocean acoustics, generally fall into two categories: source localization and environmental classification (9). This research is focused on the latter. Suppose the interface roughness parameters are defined by the vector $\mathbf{m}$. The parameters $\mathbf{m}$ can be determined from a measurement data $\mathbf{d}$ (7). All measured data has inherent noise $\mathbf{n}$, so the data $\mathbf{d}$ can be defined as:

$$
\mathbf{d}=\mathbf{d}_{0}+\mathbf{n}
$$

The dependence of $\mathbf{d}$ on the model parameters $\mathbf{m}$ is defined by a function $\mathrm{f}$ such that

$$
f(\mathbf{m})-\mathbf{d}=0
$$




\subsection{Forward Modeling}

The function $f$ in eq. 2.2 is the forward model, which is the "key factor governing the solvability of an inverse problem" (7). Forward models for wave propagation produce predictions of output signals at different source and receiver positions at some time $t$. Forward models can be formulated from observations, physics, and simulations (numerical implementation of physical models, as shown in fig. 2.1) (1).

For seabed classification the precise values of parameters is not necessary because a large range of parameter values can lead to the same classification. In other words, the classification problem can be somewhat easier than trying to obtain precise values for each of the parameters. The model used will define what parameters classify the seabed and how accurately these parameters model the environment. Acoustic propagation can, in general, be non-linear in nature which makes the forward model $f$ complicated (7). However, most models are linearized making them somewhat less realistic but more computationally feasible. In any case, model selection is based on a variety of constraints including the complexity of the classification problem and the approximations used in the formulation of the model. The parameterization as well as the approximations can sometimes cause the system to be underdetermined or overdetermined (7). Overdetermined systems have more equations than unknowns, while underdetermined systems have less equations than unknowns. Later sections will provide more details on the specific approximations and constraints. 


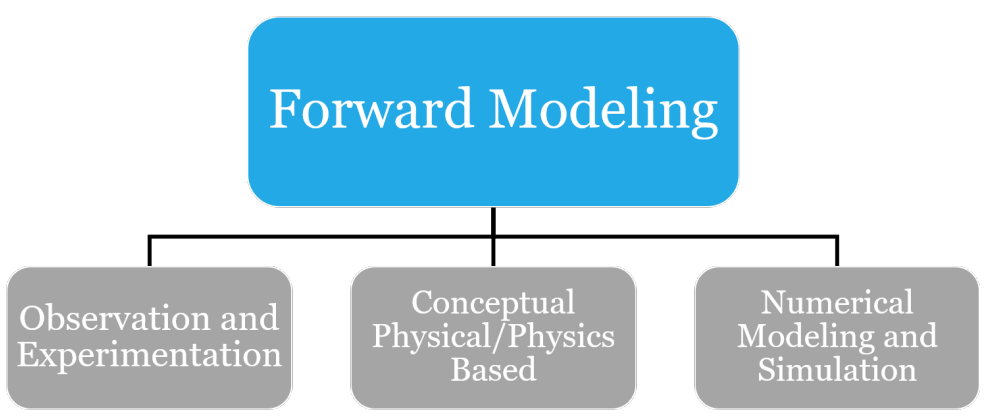

Figure 2.1: Types of forward modeling

\subsection{Inversion Methods}

After a forward has been selected selected, an inversion method is required to estimate the parameters $\mathbf{m}$. The main goal of an inversion method is to find the optimal parameters that minimize the error between the simulated and measurement data. The optimal values are found by exploring the search space (parameter values) (10). Initially, modeled signals are produced and compared to the measurement data. If the simulated signals are defined as $\hat{\mathbf{d}}=f(\mathbf{m}, t)$ and the measurement data is $\mathbf{d}$ the optimal solution would be:

$$
\mathbf{d}=\hat{\mathbf{d}}
$$

Signals are produced by the forward model is compared to the real signal using a difference metric which is defined by a cost function. The difference or error between the simulate and measurement data is minimized by the 
following process: a new model prediction is compared to the measurement data, an the error is quantified through the cost function. The result is then compared to the previous 'best' fitting value. This process is repeated over and over again until some predetermined error value is reached or all possible model predictions have been produced. This structure is shown in fig. 2.2. 


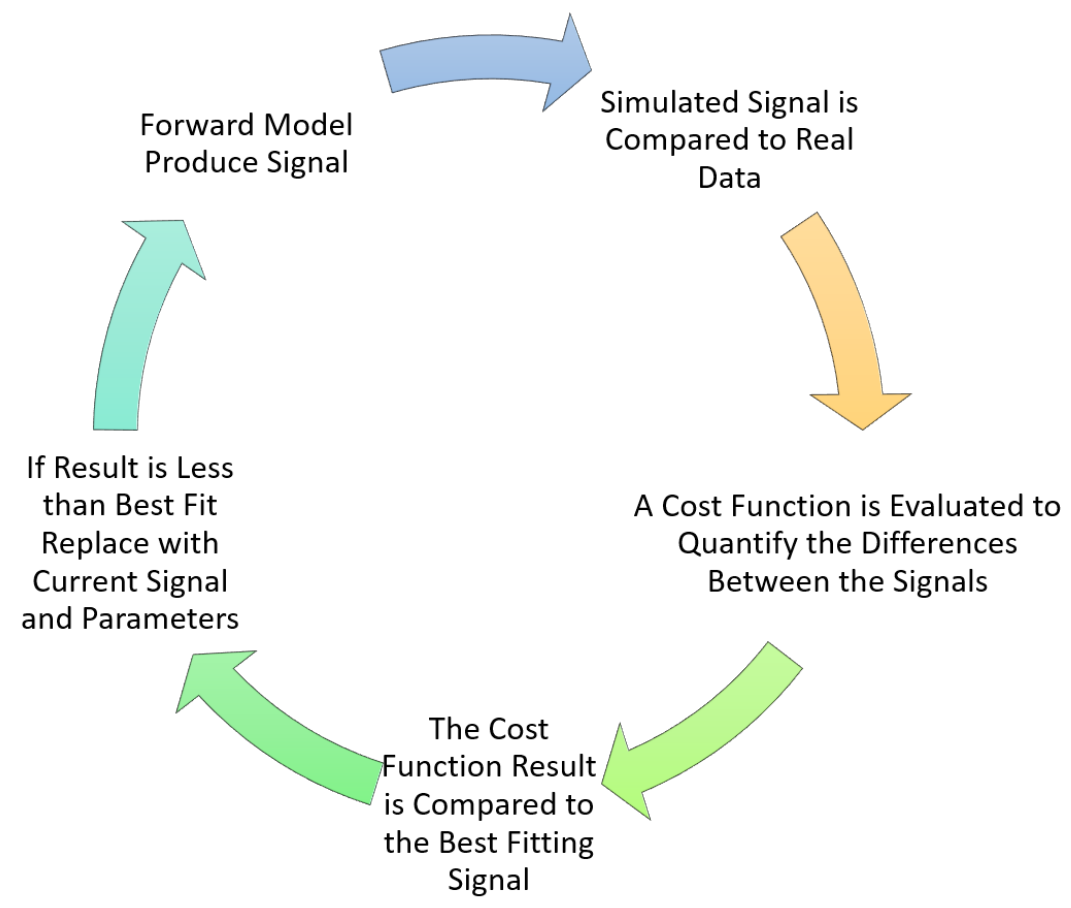

Figure 2.2: Inverse modeling cycle 


\section{Chapter 3}

\section{Background on Propagation and Scattering}

To gain a better understanding of interface scattering forward models and inverse methods, this chapter introduces wave theory and scattering.

\subsection{Wave Theory}

Fundamentally, scattering models are based on the physics of wave propagation and are solutions to the acoustic wave equation. The acoustic wave equation is derived from hydrodynamics, see (11) for derivation.

In the underwater acoustic environment, the material properties change slowly over time allowing certain parameters to be assumed constant or slowly varying. Specifically, density $\rho$ and sound speed $c$. Under this assumption the wave equation can be defined in terms of pressure $P$ :

$$
\nabla^{2} P-\frac{1}{c^{2}} \frac{\partial^{2} P}{\partial t^{2}}=0
$$

In order to simplify the solution of the wave equation, a transformation from the time domain into the frequency domain is made (11). This produces the Helmholtz equation defined in 3.2 where $\Psi$ is defined as the particle displacement potential (from which pressure can be derived), (11).

$$
\left(\nabla^{2}+k^{2}(\mathbf{r})\right) \Psi(\mathbf{r}, \omega)=0
$$




\subsubsection{Solutions to the Wave Equation}

The solution to the wave equation depends on the boundary conditions including what is being referred to in this report as the interface roughness parameters. It also depends on source-receiver geometry, frequency and bandwidth, and sound speed (11). In this research the medium of propagation is assumed to be homogeneous, resulting in a wavenumber $k$ that no longer depends on location in space, $\mathbf{r}$. This assumption leads to a simple solution to equation 3.2 in the Cartesian coordinate system. In fact, an exact integral solution can be found, which will be presented and used as reference ground truth in the model validation section.

To find a solution to the wave equation requires the Laplacian operator, which is unique to coordinate system: Cartesian, cylindrical, and spherical. In Cartesian coordinates the Laplacian operator is defined as:

$$
\nabla^{2}=\frac{\partial^{2}}{\partial x^{2}}+\frac{\partial^{2}}{\partial y^{2}}+\frac{\partial^{2}}{\partial z^{2}}
$$

In cylindrical:

$$
\nabla^{2}=\frac{1}{r} \frac{\partial}{\partial r} r \frac{\partial}{\partial r}+\frac{1}{r} \frac{\partial^{2}}{\partial \phi^{2}}+\frac{\partial^{2}}{\partial z^{2}}
$$

In spherical:

$$
\nabla^{2}=\frac{1}{r^{2}} \frac{\partial}{\partial r}\left(r^{2} \frac{\partial \Psi}{\partial r}\right)+\frac{1}{r^{2} \sin \theta} \frac{\partial}{\partial \theta}\left(\sin \theta \frac{\partial \Psi}{\partial \theta}\right)+\frac{1}{r^{2} \sin ^{2} \theta} \frac{\partial^{2} \Psi}{\partial \phi^{2}}+k^{2} \Psi
$$

Acoustic sources can be arranged in different geometries that produce dif- 
ferent solutions to the Laplacian operator. For example, in cylindrical coordinates a uniform line source reduces the Helmholtz equation to:

$$
\left[\frac{1}{r} \frac{\partial}{\partial r} r \frac{\partial}{\partial r}+k^{2}\right] \Psi(r)=0
$$

The solution can be described as Bessel functions or in terms of Hankel functions. The Bessel function solution is:

$$
\Psi(r)=\left\{\begin{array}{l}
A J_{0}(k r) \\
B J_{0}(k r)
\end{array}\right.
$$

The solution in terms of Hankel functions is:

$$
\Psi(r)=\left\{\begin{array}{l}
C H_{0}^{(1)}(k r)=C\left[J_{0}(k r)+i Y_{0}(k r)\right] \\
D H_{0}^{(2)}(k r)=D\left[J_{0}(k r)-i Y_{0}(k r)\right]
\end{array}\right.
$$

Note that $C$ represents converging waves while $D$ represents diverging waves. As $k r \rightarrow \infty$ the Hankel function is approximately equal to:

$$
H_{0}^{(1)}(k r) \approx \sqrt{\frac{2}{\pi k r}} e^{i\left(k r-\frac{\pi}{4}\right)}
$$

The Laplacian in spherical coordinates, for a point source, only depends on $r$, the distance from the source, so $\phi$ and $\theta$ in the Laplacian are 0 resulting 
in the Helmholtz equation being:

$$
\left[\frac{1}{r^{2}} \frac{\partial}{\partial r} r^{2} \frac{\partial}{\partial r}+k^{2}\right] \Psi(r)=0
$$

With the solutions:

$$
\Psi(r)=\left\{\begin{array}{l}
\frac{A}{r} e^{i k r} \\
\frac{B}{r} e^{i k r}
\end{array}\right.
$$

A summary of theoretical approaches to propagation modeling based on the wave equation is shown in Fig. 3.1. (This figure uses a slightly different notation for the wave equation but should easily translate.)

\subsubsection{Plane Wave Approximation}

A plane wave approximation can be used to reduce the complexity of solving the wave equation. Plane waves define an acoustic field that only depends on the spatial coordinate in the direction of propagation (12). This type of wavefront is not a realistic phenomena but is a useful approximation of spherical waves far from the source (13). Figures 3.2 to 3.4 illustrate a spherical source propagating over space, as the wave gets further from the source it becomes more planar in shape, explaining the practicality of this approximation (14).

However, there is a constraint on the valid region in which the plane wave approximation can be made. Depending on the frequency, The distance $\mathrm{d}$ required can be found using eq. 3.12 .

$$
f<\frac{c}{2 \mathrm{~d}}
$$




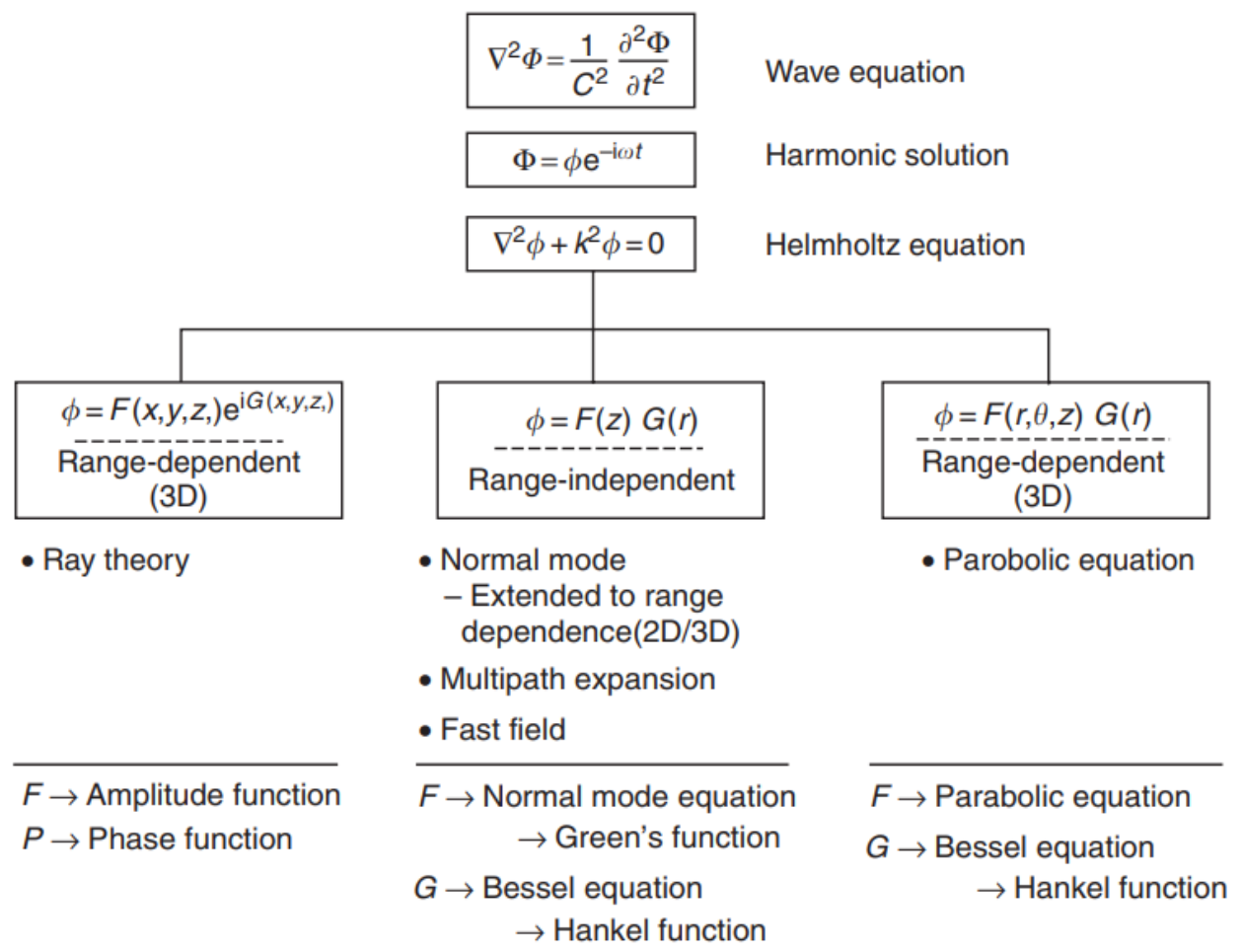

Figure 3.1: Summary of relationships among theoretical approaches for propagation modeling (Source((1)) 


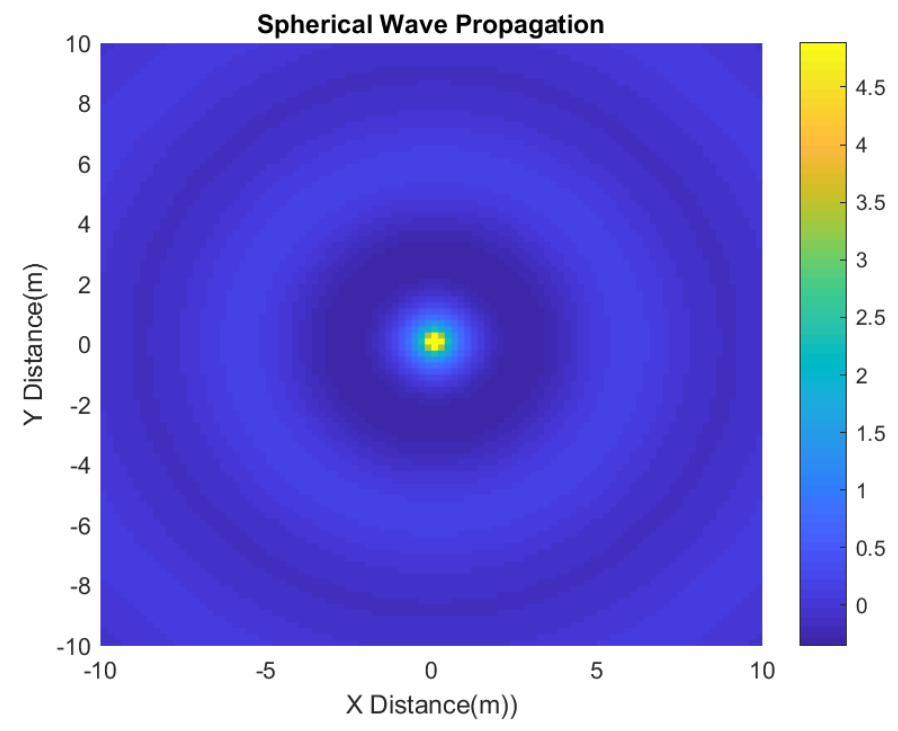

Figure 3.2: Spherical point source

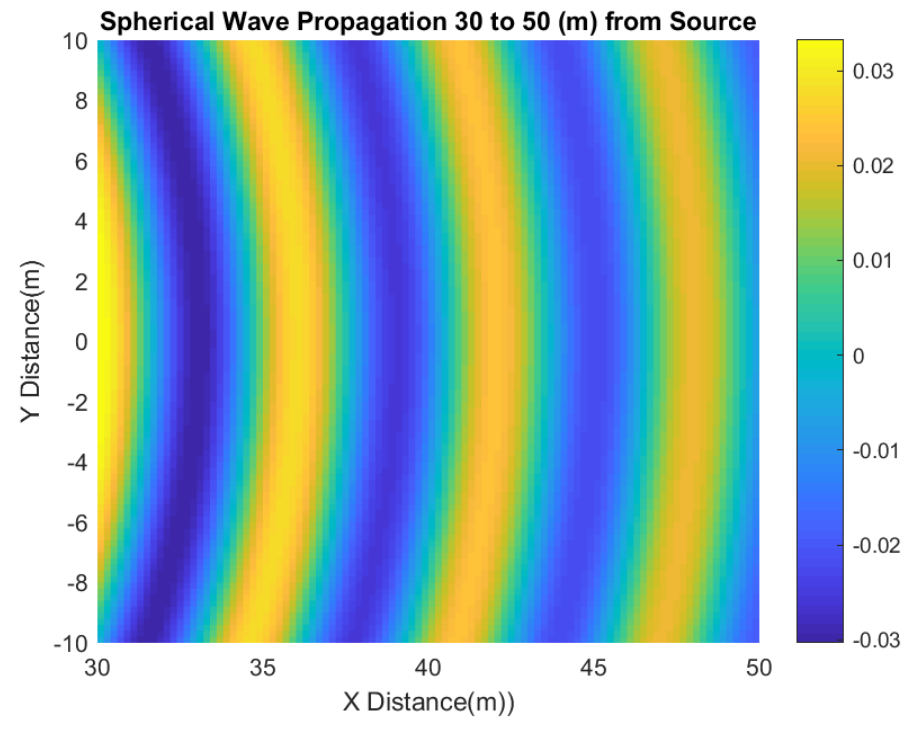

Figure 3.3: Spherical wave propagation away from point source 


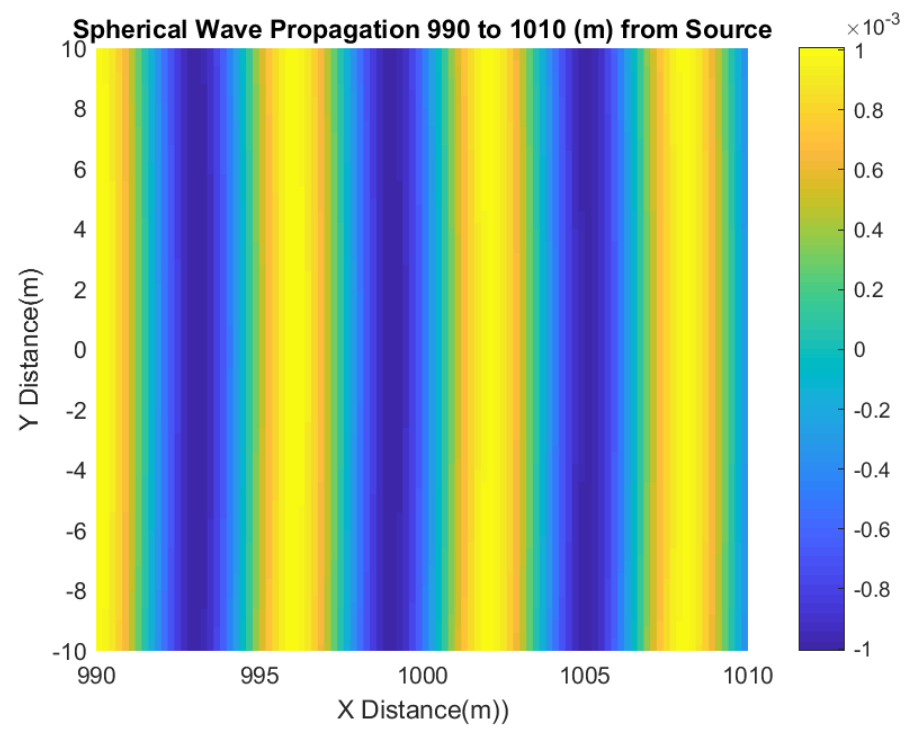

Figure 3.4: Spherical wave propagation far from point source

Under the plane wave approximation the propagation direction can be defined in terms of a single coordinate, $x$ in the Cartesian coordinate system (15). The resulting solution when $k_{y}$ and $k_{z}$ equal 0 is:

$$
\Psi(x)=\left\{\begin{array}{l}
A e^{i k x} \\
B e^{i k x}
\end{array}\right.
$$

In terms of pressure $P$ this equation can be re-written as:

$$
P(x, t)=P_{0} e^{j(\omega t-k x)} e^{-\alpha x},
$$

where $\alpha$ is a loss term. 


\subsection{Transmission Loss}

The acoustic intensity for an approximate plane wave with amplitude $P$ is given by:

$$
I=\frac{P^{2}}{2 \rho c} \text { in Watts } / \mathrm{m}^{2} .
$$

However, it is important to realize that intensity diminishes with distance (for non-planewave propagation) due to geometrical spreading, which is referred to as transmission loss: the reduction of the signal intensity over the distance traveled d (16). Transmission loss $T L$ is defined in $\mathrm{dB}$ as:

$$
T L=10 \log _{10}\left(\frac{I_{0}}{I_{r}}\right)
$$

where $I_{0}$ is the acoustic intensity at a point 1 meter from the source and $I_{r}$ is the received intensity at some receiver at a location $r$ (11). The signal loss observed is caused by spherical/cylindrical spreading that attenuates the signal as it propagates due to the source/receiver geometry (often referred to as geometric spreading loss). It can also attenuate from the $\alpha$ losses introduced earlier which is due to the medium itself.

The transmission loss from a single point source is illustrated in fig. 3.5

Note, that in environments with multiple reflections off boundaries, the transmission loss can be complicated and are not as simple as these direct propagation equations. However, these more complicated cases usually require numerical solutions. 


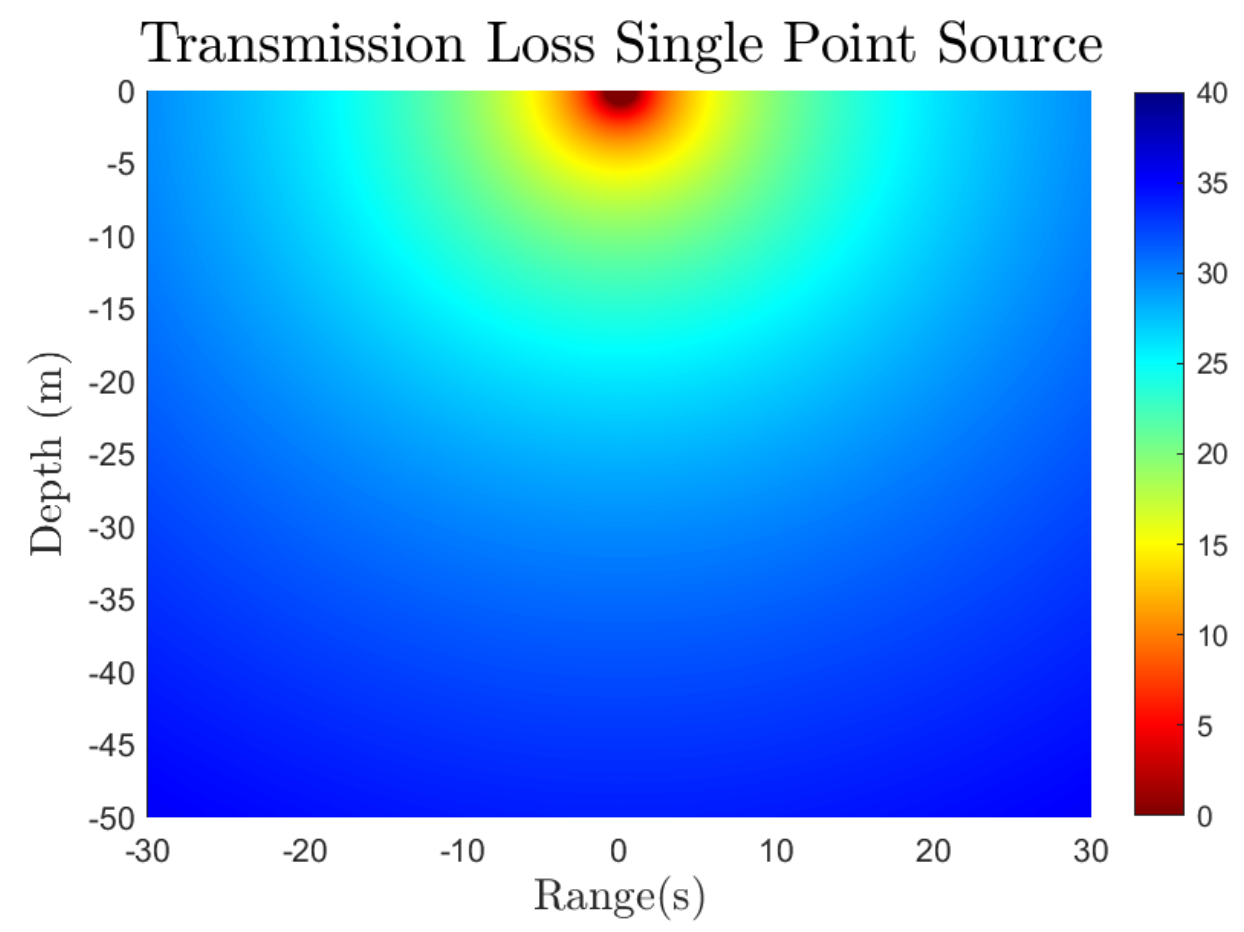

Figure 3.5: Single point source transmission loss

\subsubsection{Geometric Spreading Loss}

Spreading loss is a measure of signal weakening due to geometrical spreading of the propagating wavefronts. In underwater acoustics there are two important geometries to consider for spreading loss: spherical and cylindrical (17).

Spherical spreading, as shown in Fig. 3.6, results from a point source in a medium that is unbounded and homogeneous such that the wavefronts retain a spherical shape. The intensity of the signal decreases with distance $r$ as an inverse square (16). For a source of constant power $\mathrm{P}$ spreading loss is defined in eq. 3.17 

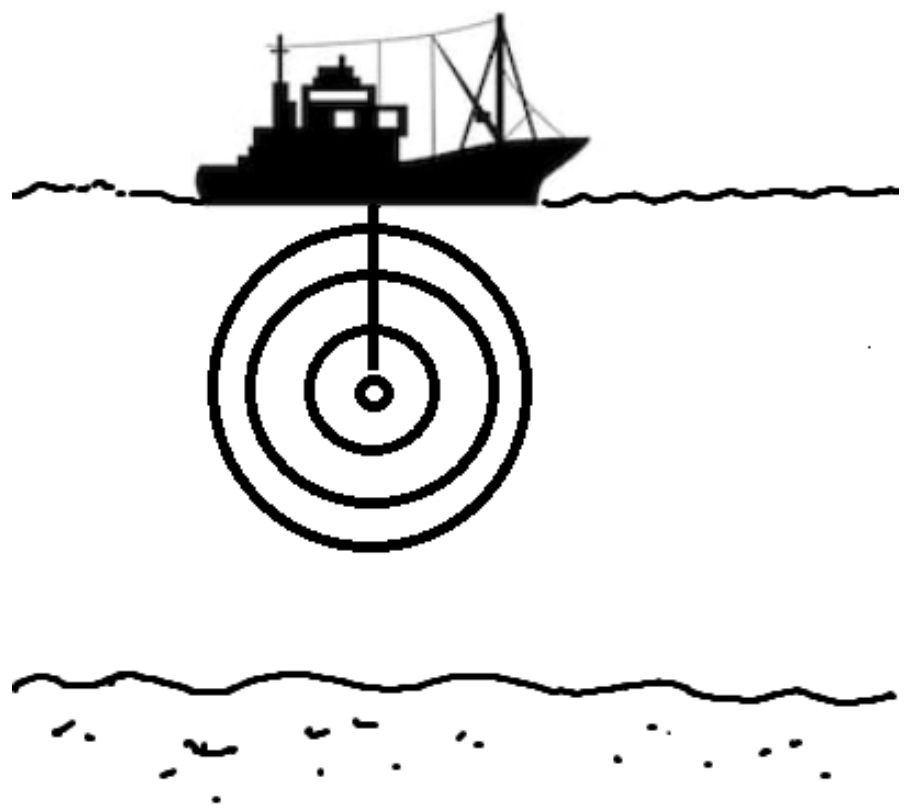

Figure 3.6: Spherical spreading from point source

$$
I(r)=\frac{\mathrm{P}}{4 \pi r^{2}}
$$

If a medium has an upper and lower boundary the wavefronts emitted from a source are often better approximated as cylindrical in shape. The intensity of these signals decreases with distance $r$ as an inverse (16). If the power $\mathrm{P}$ is constant and $d_{c}$ is the width of the cylinder the intensity is defined as:

$$
I(r)=\frac{\mathrm{P}}{2 \pi r d_{c}}
$$

However, in most cases a far field approximation is made so geometric spreading loss occurs at a rate of $\frac{1}{r}(16)$. 


\subsubsection{Attenuation}

Attenuation losses can be attributed to frequency dependent absorption and scattering (18). The wave equation, defined in eq. 3.14, incorporates the attenuation coefficient $\alpha$, which has dimensions length ${ }^{-1}$, equivalently $(\mathrm{Np} / \mathrm{m})$ (16). The attenuation coefficient can be defined as a proportional intensity change during propagation by:

$$
\frac{d I}{I}=-2 \alpha d x
$$

integration of which gives,

$$
I_{r}=I_{0} \exp \left(-2 \alpha\left(x_{r}-x_{0}\right)\right) .
$$

Attenuation from scattering is caused by, for example, inhomogenities in the seafloor caused by rocks or other irregularities.

\subsection{Scattering and Reflection}

As sound waves propagates through the ocean they are reflected, transmitted, or scattered by the surface and bottom (19). The variation of sound speed, which varies due to density, temperature, and salinity causes the waves to refract in a way similar to light through a lens (19). Analogous to the index of refraction that causes light to reflect and scatter, the characteristic impedance $\rho c$ in different rough interfaces in the ocean cause sound waves to reflect and 
scatter (1). The scattering and reflection processes are described in high level schematic shown in Fig. 3.7 .

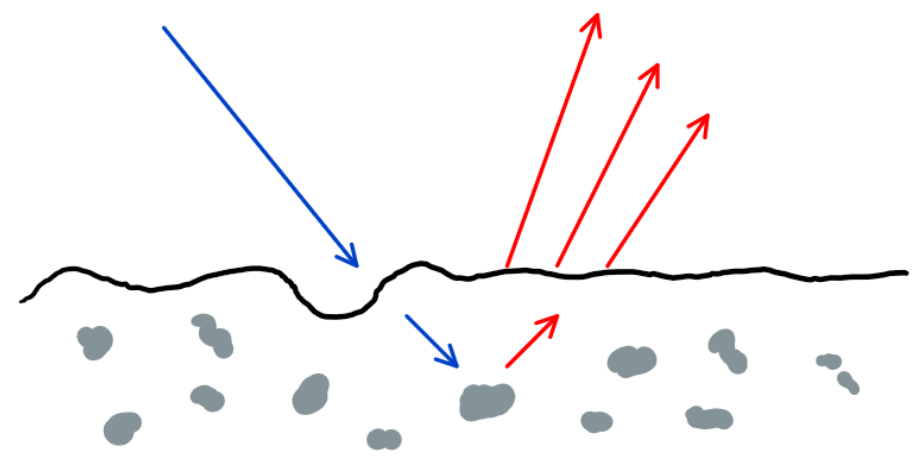

Figure 3.7: High level schematic to describe acoustic rough interface scattering (adapted from (2))

In the case of a plane wave, the reflected signal is coherently reflected in a direction symmetric with its grazing angle (18). The loss observed in the reflected signal can be quantified by the reflection coefficient, derived from Snell's Law, of the interface defined below:

$$
R=\frac{\rho_{2} k_{z, 1}-\rho_{1} k_{z, 2}}{\rho_{2} k_{z, 1}+\rho_{1} k_{z, 2}}
$$

Consider the case of a perfectly flat homogeneous medium where scattering can be ignored (1). In this idealized case, the spherical wave propagates from a source and interacts with a flat seabed causing the energy to reflect back towards the source and be partially transmitted through the boundary (1). The reflectd pressure field is defined in eq. 3.22 . Note, this equation does not 
include an attenuation factor $\alpha$. That means the reflected signal is simply the source signal with less intensity defined by the geometry of the source and receiver and the boundary loss.

$$
P=R \frac{P_{0}}{r} e^{i k r}
$$

A realistic seafloor is generally not homogeneous or flat due to its properties varying randomly in space. The amount of energy scattered is a function of density, sound speed and size of aberrations in the propagation path (e.g., roughness) (18). The frequency of the transmitted signal also affects the scattered field because the reflection coefficient can depend on frequency and the roughness appears different as acoustic wavelengths change. In particular the wavelength dictates the amount of scatter caused by a given reflector. Under the assumption that all incident power is scattered isotropically the scattering cross section for an interface is:

$$
\sigma_{s r}=4 \pi\left(\frac{I_{s c a}}{I_{i n c}}\right)
$$

where $I_{\text {sca }}$ is the intensity scattered from a unit surface measured one meter from the source (18). The scattering cross section can be defined by different parameters such as scattered intensity, or in terms of the roughness root mean squared height and correlation length, spectral strength. 


\section{Chapter 4}

\section{Scattering Modeling and Methodology}

\subsection{Motivation}

Scattering models for acoustics were investigated as far back as World War II to improve the understanding of sonar systems in support naval operations (1). Despite initial research having military applications, numerical models of the ocean as an acoustic medium have been widely published in open literature. Over time the interest in interface scattering and its applications outside the military grew. Recently, interest in remote sensing using low-frequency side scan sonar has grown (20). However, in low frequency applications, analyzing the short range acoustic propagation is dependent on source-receiver separation, source frequency, and ocean depth (11).

\subsection{Model Considerations}

\subsubsection{Systems for Classification}

Constraints and considerations in model selection are impacted by geometry and the type of source and receiver that define the physical space and propagating parameters. An acoustic source vibrating produces a wave motion that

propagates through the surrounding medium (e.g. air or water); the catalyst of wave propagation is the variation of pressure in the outside medium, and for 
this reason, sound waves are called pressure waves (18). The speed at which the acoustic wave propagates is a function of the medium, while the shape and bandwidth of the acoustic wave is determined by the source. The receivers of acoustic waves in underwater applications are typically called hydrophones: transducers that convert pressure into a voltage signal; underwater sources, on the other hand, are comprised of transducers that can be arranged in different geometries: uniform line, point, etc. (21). Often, sound sources are treated as point sources although it is common for these to not radiate uniformly in all directions but to have some inherent directionality. Sometimes, this is by design and other times simply due to the transducer geometry. The transmission of acoustic signals from a source followed by reception of the echo (reflection) off a boundary is an example of an active sonars (SOund Navigation and Ranging) (21).

Single-beam echosounders (SBES) are a type of active sonar primarily used to determine the distance to the seabed and are comprised of one or more transducers typically mounted to the hull of a ship as shown in fig. 4.1. SBES operate at near vertical incidence with a small receptive field that produce narrow beam signals at specific frequencies (21). The narrow beam pattern produced lends itself to also being used for seabed classification (22). The returned acoustic signal contains both normal incident (specular) and backscatter information from the seabed (14). This information about intensity and signal spread due to scattering can then be combined across successive points along the seabed to produce a wider area for the classification of the bottom 
type (22).

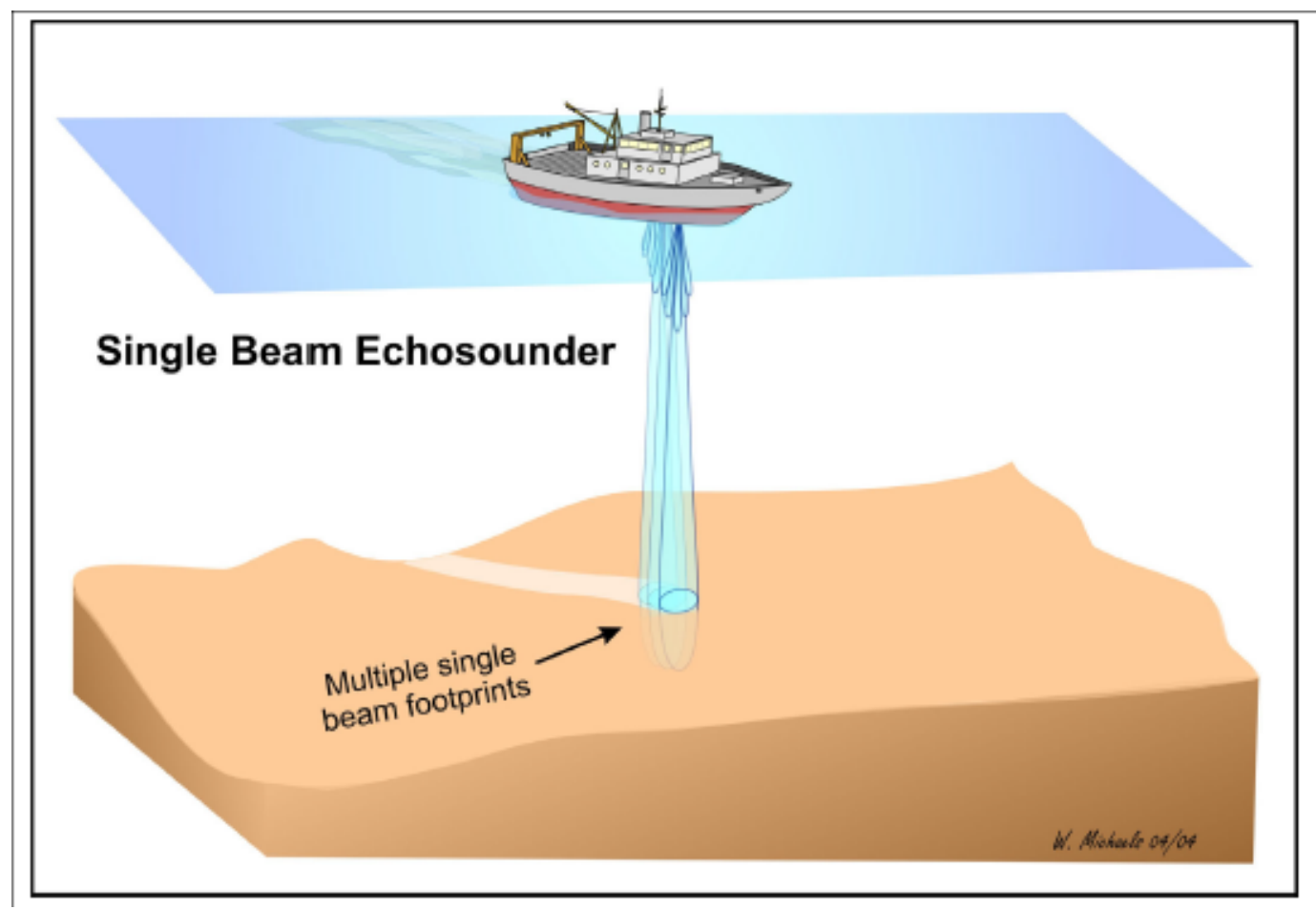

Figure 4.1: Single beam echosounder (Source (3))

Sidescan sonar (SSS) are another active sonar typically used to image rather than classify the type of the seabed (22). This acoustic system can be mounted on AUVs or towed close to the bottom with transducers on each side. The sonar transmits in a nearly horizontal direction that sweeps the bottom (21), as shown in fig. 4.2. This means the receptive field of the SSS is larger than the SBES since the acoustic signal covers a larger ensonified area due to the geometry of the source and receiver. An image is formed from a returned echo using the intensity of the returned signal over multiple pulses in time (14). 
These types of acoustic systems are generally more useful in the detection of small isolated features such as coral and rocks (22). However, these features can also dictate what bottom type and interface roughness parameters are used for seabed classification. To accomplish this, again the normally incident signal is extracted from the SSS but the processing requires an array of hydrophones so that the received signal can be beamformed (spatially filtered) to extract the normal incident returns.

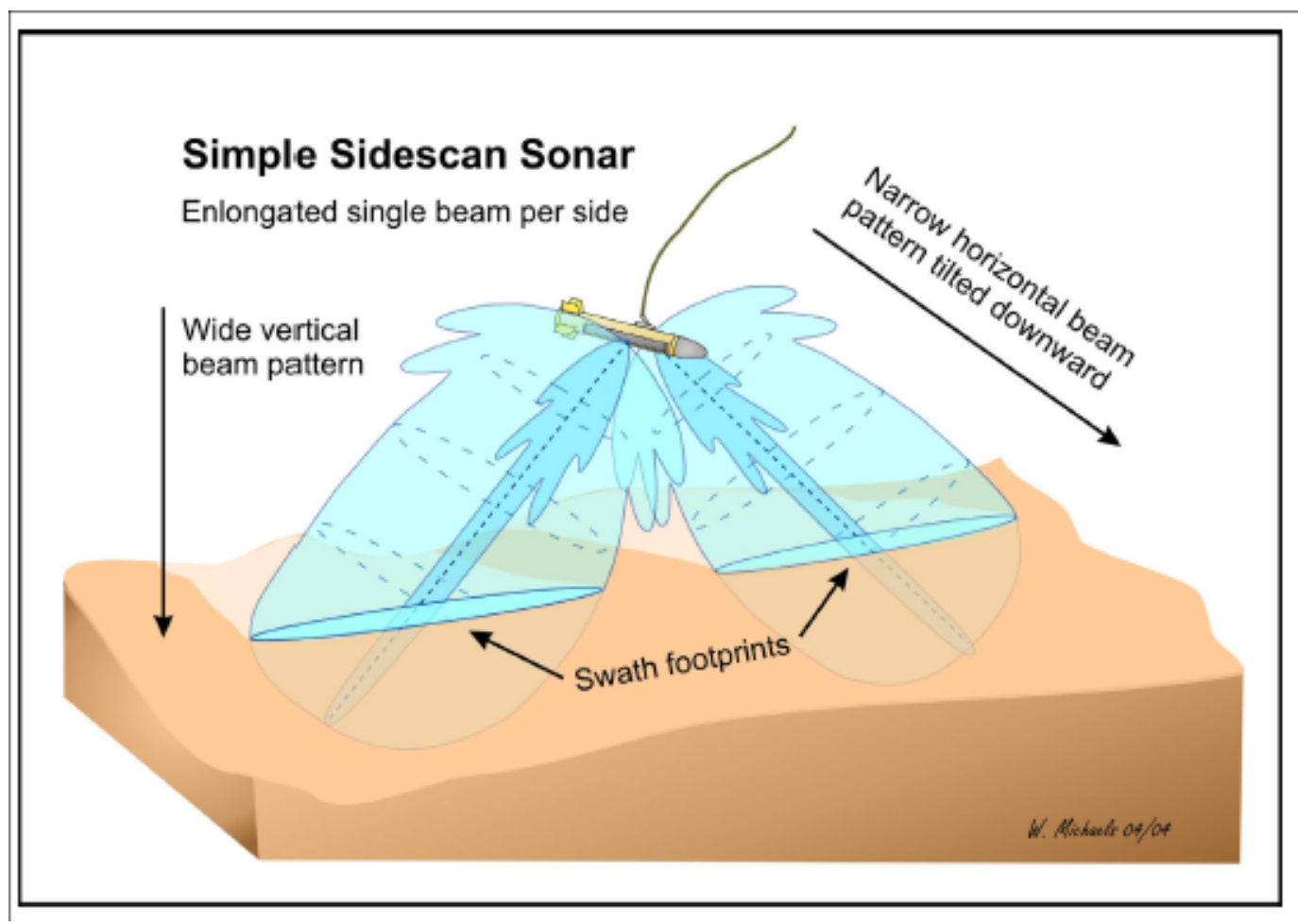

Figure 4.2: Side scan sonar (Source (3) )

Both the SBES and SSS acoustic systems perform differently for classification and typically operate at different frequencies. The frequency range of 
the signal places constraints on the valid regions of scattering approximations, since the frequency range is directly linked to the wavelength. In particular, low frequency signals can have longer wavelengths therefore "see" the rms height and slope of the seabed differently than shorter wavelength signals. Further, lower frequency signals can penetrate deeper into the seabed possibly scattering from different features in the seabed compared to a higher frequency signal. The scattering models that will be presented use approximations that require the ratio of wavelength to seabed rms height and correlation length to be within a constrained range of values. One of the issues considered here is the wide range of frequencies that may be needed for this application.

\subsubsection{Model Approximations}

Determining if the forward model can be used within a frequency range of interest is dependent upon the approximations used in the model.

\section{Kirchhoff Approximation}

The Kirchhoff approximation applies to surfaces having arbitrary height and slope but requires a local radius of curvature $R_{c}$ that satisfies:

$$
R_{c} \leq \frac{\lambda}{\pi \sin ^{3} \theta_{g}}
$$

where $\theta_{g}$ is the grazing angle of the acoustic wave on the seabed (23).

This approximation is also called the tangent plane approximation since the region ensonified is considered locally planar (4). The structure function 
$D$ corresponding to the isotropic spatial spectrum $W(24)$,

$$
W(K)=\frac{w_{2}}{K \gamma_{2}}
$$

is given by,

$$
D(\mathbf{r})=C_{h}^{2} r^{2 \alpha}
$$

Where,

$$
C_{h}^{2}=\frac{2 \pi w_{2} \Gamma(2-\alpha) 2^{-2 \alpha}}{h_{0}^{\gamma} \alpha(1-\alpha) \Gamma(1+\alpha)}
$$

and

$$
\alpha=\frac{\gamma}{2}-1
$$

Note that $\Gamma$ is the gamma function and the $\alpha$ here is unrelated to the attenuation of the propagating wave described earlier (the notation used is to be consistent with the published literature but the context should make it clear which value is represented). Parameters derived from the structure function parameters $\alpha$ and $C_{h}^{2}$

$$
\begin{gathered}
q_{c}=C_{h}^{2} 2^{1-2 \alpha} k^{2(1-\alpha)} \\
a=\left(\frac{8 \alpha^{2} \Gamma\left(\frac{1}{2 \alpha}+\frac{1}{2}\right)}{\Gamma\left(\frac{1}{2}\right) \Gamma\left(\frac{1}{\alpha}\right) \Gamma\left(\frac{1}{2 \alpha}\right)}\right)^{2 \alpha} \\
b=\frac{a^{\frac{1}{2}-\frac{1}{2 \alpha}} \Gamma\left(\frac{1}{\alpha}\right)}{2 \alpha}
\end{gathered}
$$


The resulting approximation for the Kirchhoff backscattering cross section $\sigma_{k r}(\theta)$ is,

$$
\sigma_{k r}(\theta)= \begin{cases}\frac{b q_{c}|R(90)|^{2}}{8 \pi\left[\cos ^{4 \alpha}(\theta)+a q_{c}^{2} \sin ^{4}(\theta)\right]^{\frac{1+\alpha}{2 \alpha}},} & \theta \geq 40 \\ 0 & \theta<40\end{cases}
$$

It is important to note that this approximation is valid for near normal incident angles and small RMS slope (25). This approximation is commonly used, since the seabed can generally be assumed to be smooth, with a root mean squared slope that rarely exceeds 10 degrees (11).

\section{Rayleigh-Rice Approximation}

The Rayleigh-Rice Perturbation method models the scattered field as superimposed plane waves (26). This type of field is assumed to be valid arbitrarily close to the rough interface. The bottom backscattering cross section in the Rayleigh-Rice perturbation approximation defined as:

$$
\sigma_{p r}=4 k^{4} \sin ^{4}(\theta)|Y(\theta)|^{2} W_{2}\left(K_{\theta}\right)
$$

In this expression $Y(\theta)$ is the complex function

$$
Y(\theta)=\frac{(\rho-1)^{2} \cos ^{2}(\theta)+\rho^{2}-\kappa^{2}}{[\rho \sin (\theta)+P(\theta)]^{2}}
$$


where $P(\theta)$ and $\kappa$ (the complex wave number ration) are defined as:

$$
\begin{gathered}
\kappa=\frac{1}{v}(1+i \delta) \\
P(\theta)=\sqrt{\kappa^{2}-\cos ^{2} \theta}
\end{gathered}
$$

The power spectrum $W_{2}$, for random bottom relief appears in Eq. (27).

$$
W_{2}(\mathbf{K})=\frac{w_{2}}{\left(h_{0} \mathbf{K}\right)^{-\gamma}}
$$

It is evaluated at the wavenumber

$$
K_{\theta}=\left[4 k^{2} \cos ^{2}(\theta)+\left(\frac{k}{10}\right)^{2}\right]^{\frac{1}{2}}
$$

The term involving $\frac{k}{10}$ has been added to the usual expression for the argument of the spectrum. This is done to avoid the singularity in the power-law spectrum at zero wavenumber (i.e, as $\theta \rightarrow 90$ ); this is somewhat arbitrary modification of the usual small-roughness expression only affects the cross section for grazing angles near 90, and these angles are unimportant owing to the interpolation scheme used.

\section{Born Approximation}

The assumption that the acoustic wave scatters only once before forming the scattered wave is referred to as the Born approximation (15). The interaction 
between the incoming wave at some direction $\mathbf{r}$ is deflected in a new direction $\mathbf{r}^{\prime}$ while retaining its amplitude and frequency. Allowing a spatial approximation of Green's function is defined as:

$$
G\left(\mathbf{r}-\mathbf{r}^{\prime}\right) \approx-\frac{e^{i k \mathbf{r}}}{4 \pi \mathbf{r}} e^{-i k \mathbf{r}^{\prime}}
$$

More detail on Green's functions and the Born approximation are given in the chapter dealing with the forward modeling.

\subsection{Seabed Parameters}

The seabed parameters that are being solved for are defined in this section. In addition to the usual medium parameter such as sound speed and density, the interface roughness (or scattering) parameters also need to be defined. Depending on the type of interface roughness and the forward model the roughness parameters can be defined in terms of rms height and correlation length or spectral strength and exponent. These parameters directly affect the strength and duration of the echoed (backscattered) signal (20). As previously discussed, the models of interest will assume constant density and sound speed. This is justified since the frequencies considered here are relatively high and the seabed penetration depth relatively low.

Below is a list of seabed parameters:

- $h$ : Root mean squared height of interface

- L: Correlation length of interface roughness 
- $v$ : Sound speed ratio between two mediums

- $\rho$ : Density ratio between two mediums

- $\delta$ : Ratio of imaginary wavenumber to real wavenumber

- $w_{2}$ : Strength of bottom relief spectrum (2D)

- $\gamma_{2}$ : Exponent of bottom relief spectrum (2D)

Examples of bottom types and parameter values is shown in table 4.1 .

\subsection{Seabed Classification}

Although the seabed parameters are being estimated, the objective is seabed classification which allows some error in how accurately the parameters are estimated. This is shown in Table 4.1 as there is a range of values for each parameter that results in the same seabed classification. In this research the inverse method will find the best fitting parameters and produce a seabed type based on the return values. The seabed type classification will be split into three classes: silt, sand and gravel. The roughness type will also be classified as either smooth, moderate or rough. This classification is based on a simple but useful scheme developed by the US Navy to characterize seabed types for various operations. The roughness characterization has some subtleties however. In other words, one type of roughness isn't always the same (even statistically) as another type even if the RMS height is the same. There are different types of rough surfaces that will be discussed in in the next chapter and depending on the type, the surface parameters will be different. 


\begin{tabular}{|c|c|c|c|}
\hline \multicolumn{4}{|c|}{ Parameterization of Sediment Types } \\
\hline $\begin{array}{l}\text { Sediment } \\
\text { Name }\end{array}$ & $\begin{array}{c}\text { Density Ratio } \\
\rho\end{array}$ & $\begin{array}{c}\text { Sound Speed } \\
\text { Ratio } \\
v\end{array}$ & $\begin{array}{c}\text { Spectral Strength } \\
w_{2} \\
\left(\mathrm{~cm}^{4}\right)\end{array}$ \\
\hline Clay & 1.14876 & 0.9801043 & $\begin{array}{l}5.175 \times 10^{4} \\
51.75 \times 10^{4}\end{array}$ \\
\hline Silt & 1.149182 & 0.981798 & $\begin{array}{l}5.175 \times 10^{4} \\
51.75 \times 10^{4}\end{array}$ \\
\hline Fine Sand & 1.615902 & 1.139692 & 0.0035 \\
\hline Medium Sand & 2.151217 & 1.74087 & 0.00558833 \\
\hline Coarse Sand & 2.313 & 1.2278 & 0.00860511 \\
\hline $\begin{array}{c}\text { Very Coarse } \\
\text { Sand }\end{array}$ & 2.492159 & 1.286925 & $\begin{array}{l}0.012935 \\
0.026\end{array}$ \\
\hline Gravel & 2.5 & 1.5 & 0.014 \\
\hline Rock & 2.5 & 2.5 & $\begin{array}{c}0.01862 \\
0.0518 \\
0.20693\end{array}$ \\
\hline
\end{tabular}

Table 4.1: Table of General Sediment Parameter Values (adapted from (4)) 


\section{Chapter 5}

\section{Random Rough Surfaces}

The modeled echo envelope of acoustic signals are dependent on the propagation medium and interface boundaries, which define the roughness parameters. In order to model this phenomenon, random rough surfaces are generated. These surfaces are random processes that represent the variability and uncertainty in a realistic seabed (27). The roughness distribution is described statistically through a probability density function which is defined by the dis-

placement from a surface position, the surface slopes, and the temporal and spatial spectra of the surface in combination with the correlation function(18).

\subsection{Gaussian Roughness Spectrum}

In underwater acoustics an often made assumption is that the roughness spectrum can be approximated using a Gaussian distribution (18). Gaussian rough surfaces are generated from a Gaussian spectrum that is calculated using rms height $(h)$ and correlation length $(l)(28)$.

A Gaussian one dimensional roughness spectrum is given by,

$$
W\left(K_{x}\right)=\frac{L h^{2}}{2 \sqrt{\pi}} e^{\frac{-k_{x}^{2} L^{2}}{4}} .
$$

In Fig. 5.1 a Gaussian rough surface is displayed using an rms height of $1.5 \mathrm{~cm}$ and a correlation length of $0.5 \mathrm{~m}$. The surface has a Gaussian 
curvature resulting in smoothed edges. The appeal of the Gaussian processes is that they can be completely represented through their first two moments -mean and variance (27). Due to this, Gaussian rough surfaces can be easily generated to give a somewhat realistic rough surface.

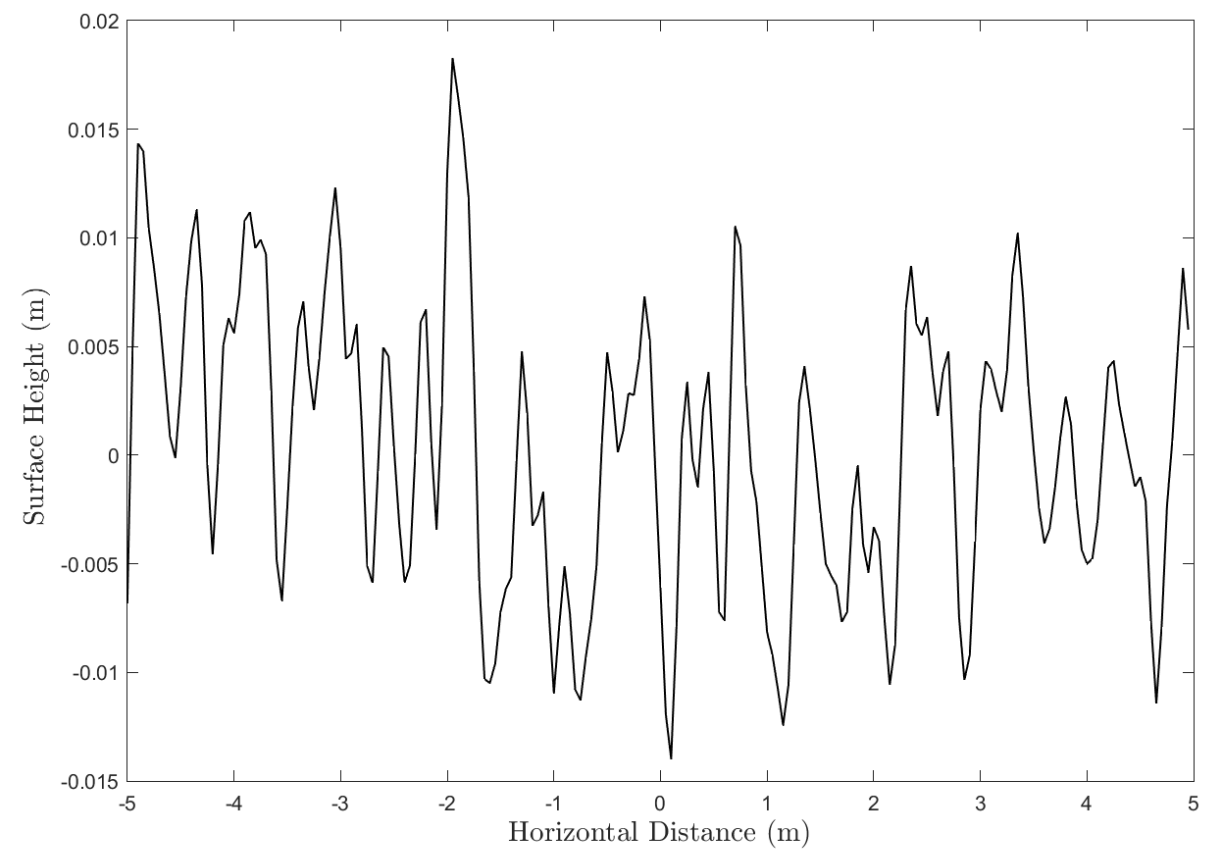

Figure 5.1: Gaussian random surface

\section{2 von-Karman Roughness Spectrum}

The von Karman Spectral form is another random surface that is sometimes used in scattering models (2) and thought of as being somewhat more representative of true seabeds. The von-Karman spectrum is defined by the bottom relief spectral exponent $\gamma$, the strength of the bottom relief spectrum $w$ and 
complex wavenumber $K$. The one dimensional spectrum is defined as (4):

$$
W\left(K_{x}\right)=\frac{w_{1}}{\left(K_{x}^{2}+\frac{1}{L}^{2}\right)^{\frac{\gamma_{1}}{2}}}
$$

To transform from the one dimensional case to the two dimensional case $w_{1}$ and $w_{2}$ are related through the following equations.

First find $\gamma_{2}$

$$
\begin{gathered}
\gamma_{2}=\gamma_{1}+1 \\
w_{2}=w_{1} * \frac{\Gamma\left(\frac{\gamma_{2}}{2}\right)}{\sqrt{\pi} \Gamma\left(\frac{\gamma_{2}-1}{2}\right)}
\end{gathered}
$$

Below is the equation for the two dimensional von-Karman spectrum:

$$
W(\mathbf{K})=\frac{w_{2}}{\left(K^{2}+K_{0}^{2}\right)^{\frac{\gamma_{2}}{2}}}
$$

The mean-squared roughness height in this case is

$$
h^{2}=\frac{2 \pi w_{2}}{\left(\gamma_{2}-2\right) K_{0}^{\gamma_{2}-2}}
$$

The von Karman spectrum approaches a constant value as wavenumber approaches zero, and for large wavenumbers $\left(K>>K_{0}\right)$ approaches zero as an inverse power of wavenumber (2). This phenomenon is shown in fig. 5.2 .

The roughness spectrum is also assumed to follow the simple power law, which simplifies the spectral equation. Based on the simplified von Karman spectrum defined in eq. 5.7, it can be shown that spectrum only depends on the 


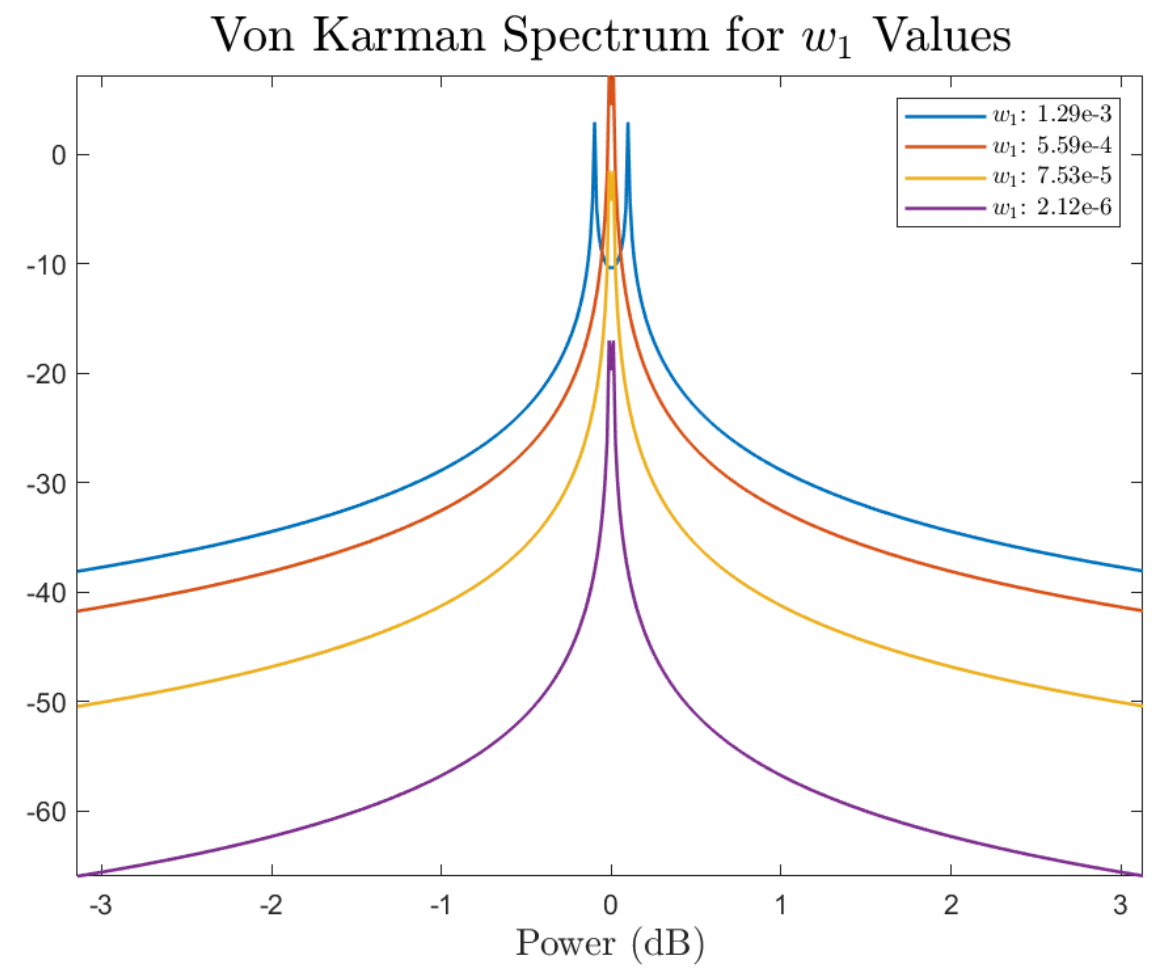

Figure 5.2: von Karman spectrum 
magnitude, $K$. This comes from the assumption that the seafloor roughness is isotropic.

$$
W(K)=\frac{w_{2}}{K^{\gamma_{2}}}
$$

The rough surface created using the von Karman spectrum, shown in fig. 5.3 , is a more realistic interface than a Gaussian random surface since it combines sharp and smooth edges.

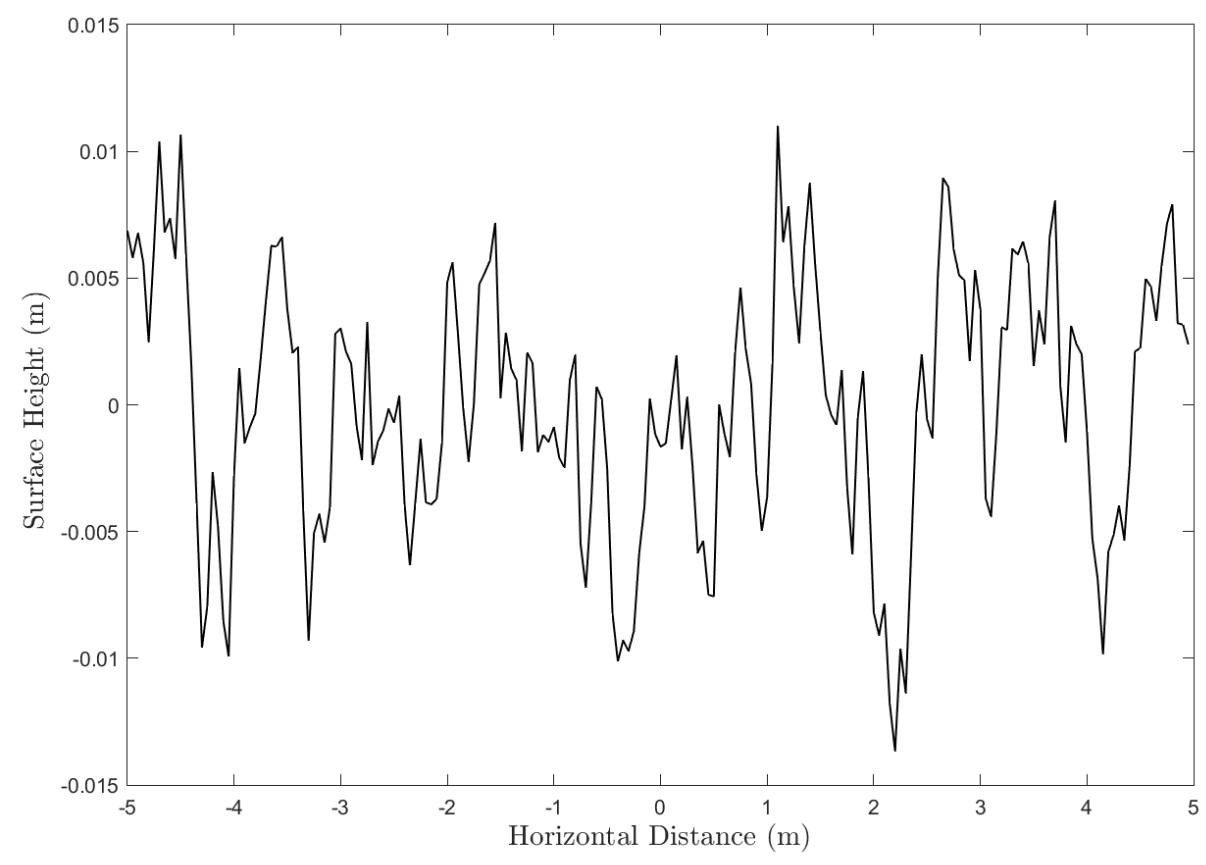

Figure 5.3: von Karman random surface 


\section{Chapter 6}

\section{Numerical Model Theory and Validation}

There are many forward models that have been developed for underwater acoustics application and these have been defined and discussed in detail (see for example, (11)). The model being proposed for this work is ray based and develped by Pinson and Cordioli (4). This section will present the PinsonCorioli model along with two others for comparison and validation.

\subsection{Pinson-Cordioli Model}

The Pinson-Cordioli model was developed to study the influence seabed roughness has on sound speed profile measurement uncertainties (4). This was mainly to improve results from bathymetric surveys. However, in this research the model is used as the forward model in the inversion method to classify the seabed.

The Pinson-Cordioli model is also a three dimensional model that was conceived based on the inhomogeneous wave equation using Green's function and the ray approximation. More information on ray theory and modeling is presented in (11) and (21).

This model can be applied to layered and three dimensional environments, which differs from other wave propagation models. This model defines $\Psi(\mathbf{r}, \omega)$ as in eq. 3.2. Including a spatial point source (delta function $\delta$, the Helmholtz 
equation becomes:

$$
\nabla^{2} \Psi+k^{2} \Psi=-\sum_{a=1}^{N e s} S_{a} \delta\left(\mathbf{r}-\mathbf{r}_{a}\right)
$$

where $\Psi$ is the displacement potential, $N_{e s}$ is the number of sources, and $S_{a}$ is the magnitude of the source signal, $\mathbf{r}$ is the receiver location, and $\mathbf{r}_{a}$ is the source position (4).

In order to solve the wave equation, the Born and Kirchhoff approximations are applied. From the Born approximation for a finite homogeneous medium Green's Theorem can be applied.

$$
\begin{gathered}
\Delta G\left(\mathbf{r}, \mathbf{r}^{\prime}\right)+\left(\frac{\omega}{c}\right)^{2} G\left(\mathbf{r}, \mathbf{r}^{\prime}\right)=-\delta\left(\mathbf{r}, \mathbf{r}^{\prime}\right) \\
G\left(\mathbf{r}, \mathbf{r}^{\prime}\right)=\frac{e^{i k\left|\mathbf{r}-\mathbf{r}^{\prime}\right|}}{4 \pi\left|\mathbf{r}-\mathbf{r}^{\prime}\right|}
\end{gathered}
$$

The derivation of this model results in the following equation:

$$
P(\mathbf{r})=4 \pi S G\left(\mathbf{r}, \mathbf{r}^{\prime}\right)+I^{i}(\mathbf{r})+I^{r}(\mathbf{r})+I^{s}(\mathbf{r})
$$

where

$$
I^{i}(\mathbf{r})=\int\left(G\left(\mathbf{r}, \mathbf{r}^{\prime}\right) \nabla_{\mathbf{n}} P^{i}\left(\mathbf{r}^{\prime}\right)-P^{i}\left(\mathbf{r}^{\prime}\right) \nabla_{\mathbf{n}} G\left(\mathbf{r}^{\prime}, \mathbf{r}\right)\right) \mathrm{d} \mathbf{r}^{\prime}=0
$$




$$
\begin{gathered}
I^{r}(\mathbf{r})=-\int\left(G\left(\mathbf{r}, \mathbf{r}^{\prime}\right) R_{01} \nabla_{\mathbf{n}} P^{i}\left(\mathbf{r}^{\prime}\right)+R_{01} P^{i}\left(\mathbf{r}^{\prime}\right) \nabla_{\mathbf{n}} G\left(\mathbf{r}, \mathbf{r}^{\prime}\right)\right) \mathrm{d} \mathbf{r}^{\prime} \\
I^{s}(\mathbf{r})=\int\left(G\left(\mathbf{r}, \mathbf{r}^{\prime}\right) T_{10} \nabla_{\mathbf{n}} P^{s}\left(\mathbf{r}^{\prime}\right)-T_{10} P^{s}\left(\mathbf{r}^{\prime}\right) \nabla_{\mathbf{n}} G\left(\mathbf{r}, \mathbf{r}^{\prime}\right)\right) \mathrm{d} \mathbf{r}^{\prime}
\end{gathered}
$$

where $R_{01}$ is the reflection coefficient, $T_{10}$ is the transmission coefficient from one interface to another.

The geometry of the seabed in this model is shown in figure 6.1.

In order to numerically evaluate this model, ray paths need to be calculated based on the Langston method (29) outlined below:

1. Send a ray parameterized by the angles $\theta_{0}$ and $\psi_{0}$ from the source to the receiver.

2. Find the intersection point of this ray on the next surface.

3. Using Snell's equation, calculate the transmission coefficient.

4. Iterate over all interfaces.

5. Determine reflection coefficient of the surface.(4) 


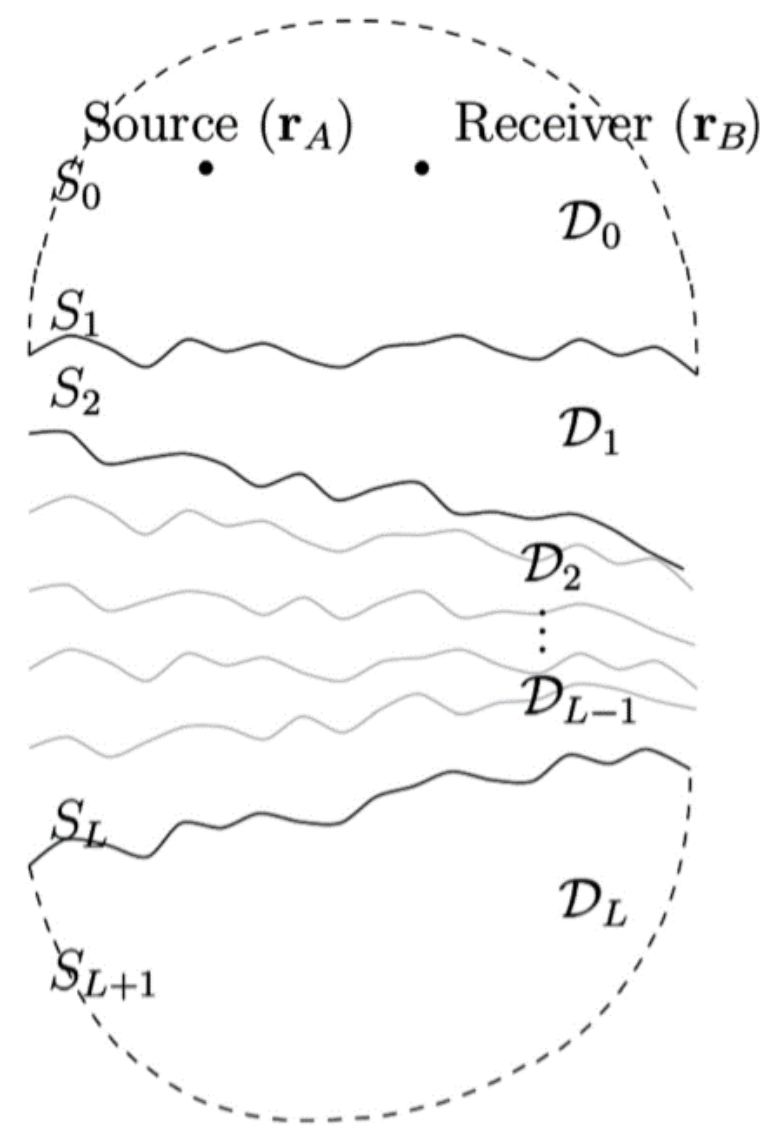

Figure 6.1: Pinson model layered rough interface geometry (Source (4))

\subsection{Exact integral solution}

For validating the Pinson model, an exact solution to 1D scattering has been determined in Cartesian coordinates using the Helmholtz-Kirchhoff integral equation (30). The geometric interpretation of scattering for the exact integral equation is shown in figure 6.2. The boundary is assumed to be a pressure release surface that falls off smoothly near the ends to avoid edge affects (30). 
The Helmholtz integral formula defines the total acoustic pressure $P(\mathbf{r})$ that results from an incident field $P_{\text {inc }}$ and a scattered field from a surface $S$ as:

$$
P(\bar{r})=P_{\mathrm{inc}}(\bar{r})-\frac{1}{4 i} \int_{s} H_{0}^{(1)}\left(k\left(\left|\bar{r}-\bar{r}^{\prime}\right|\right) \frac{\partial P\left(\bar{r}^{\prime}\right)}{\partial n^{\prime}} d s^{\prime}\right.
$$

In eq. $6.8 \frac{\partial \mathbf{r}}{\partial n^{\prime}}$ is the undetermined normal derivative of the total pressure on the surface. This derivative can be described by two integral equations by letting $\mathbf{r}$ approach the pressure release boundary giving:

$$
P_{\mathrm{inc}}(\bar{r})=\frac{1}{4 i} \int_{s} H_{0}^{(1)}\left(k\left(\left|\bar{r}-\bar{r}^{\prime}\right|\right) \frac{\partial P\left(\bar{r}^{\prime}\right)}{\partial n^{\prime}} d s^{\prime}\right.
$$

and

$$
\frac{\partial P(\bar{r})}{\partial n}=2 \frac{\partial P_{\mathrm{inc}}(\bar{r})}{\partial n}-\frac{1}{2 i} \int_{s} \frac{\partial}{\partial n} \times H_{0}^{(1)}\left(k\left|\bar{r}-\bar{r}^{\prime}\right|\right) \frac{\partial \bar{r}^{\prime}}{\partial n^{\prime}} d s^{\prime}
$$

Once the normal derivative has been calculated via eq. 6.9 or eq. 6.10 the scattered field can be calculated using eq. 6.11.

$$
P_{s}(\bar{r})=P(\bar{r})-P_{\text {inc }}(\bar{r})
$$

where,

$$
\begin{gathered}
P_{\text {inc }}=\exp \left(i \bar{k}_{\text {inc }} \cdot \bar{r}[1+w(\bar{r})]-\frac{(x-z \cot \theta)^{2}}{g^{2}}\right) \\
w(\bar{r})=\frac{\frac{2(x-z \cot \theta)^{2}}{g}-1}{(k g \sin \theta)^{2}}
\end{gathered}
$$




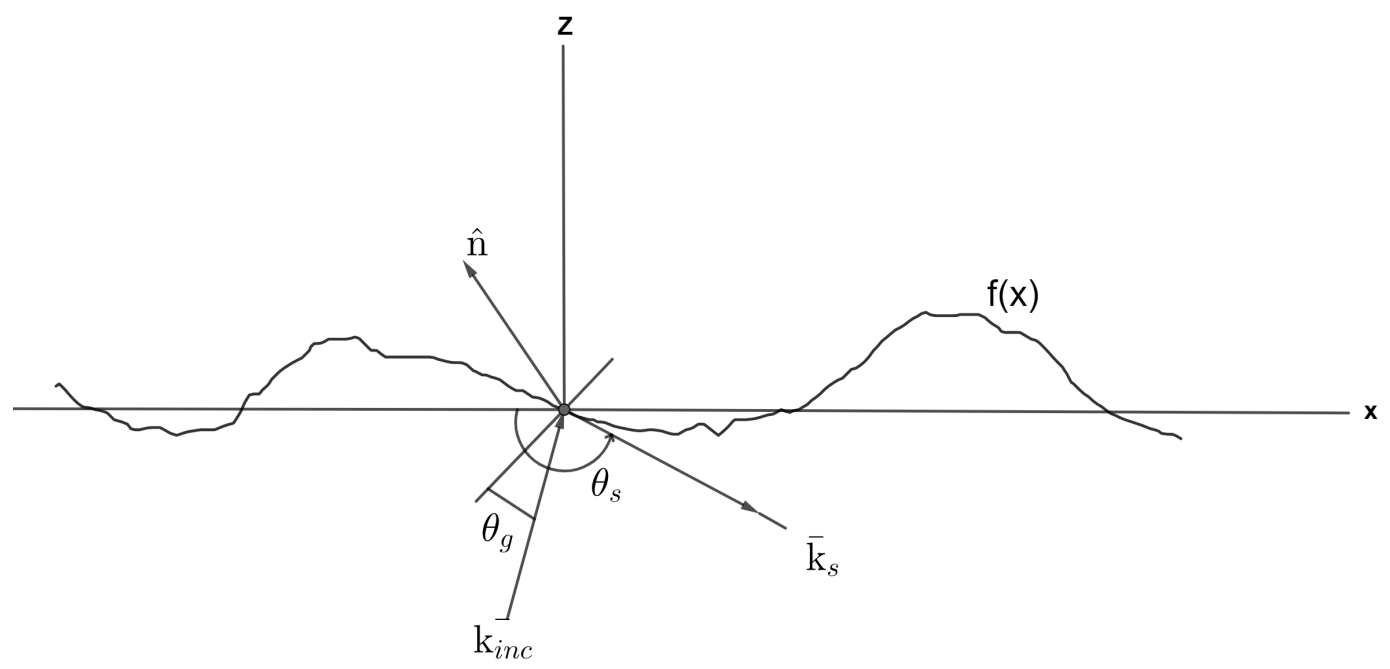

Figure 6.2: Geometry of exact integral equation

$$
\begin{gathered}
H_{0}^{(1)}\left(k\left|\bar{r}-\bar{r}^{\prime}\right|\right) \sim\left(\frac{2}{\pi k}\right)^{\frac{1}{2}} e^{-\frac{i \pi}{4}}\left(\frac{e^{i k r}}{\sqrt{r}}\right) e^{-\bar{k}_{s} \cdot \bar{r}^{\prime}} \\
P_{s}(\bar{r})=-\left(\frac{2}{\pi k}\right)^{\frac{1}{2}} e^{-\frac{i \pi}{4}}\left(\frac{e^{i k r}}{\sqrt{r}}\right) \sum_{n=1}^{N} e^{-\bar{k}_{s} \cdot \bar{r}_{n}} b_{n} \\
b_{n}=\left.\frac{\Delta x}{4 i} \gamma_{n} \frac{\partial p\left(\bar{r}^{\prime}\right)}{\partial n^{\prime}}\right|^{r_{n}} \\
\Delta x=\frac{D}{N}
\end{gathered}
$$

$N$ number of surface partitions and D is the total surface length.

Since this model integrates over the entire acoustic field it is computa- 
tionally complex and sensitive to the field and grid used in the simulation. The exact integral model uses a plane wave source in Cartesian coordinates, however, this source will be changed into a spherical point source for direct comparison with the Pinson model.

\subsection{The Snellen, Siemes, and Simon Model}

Single beam echosounders (SBES) are being widely used to classify sediment properties. The Snellen Siemes Simon Model, which will be referred to as the Snellen models a SBES echo envelope (5). Modeling of the echo envelope is based on interface and volume scattering using the Rayleigh-Rice perturbation approximation. The received SBES signal is modeled as:

$$
\mathrm{y}(t)=\int_{A(t)} \sigma_{b}(\theta) B(\theta) \frac{e^{-4 \alpha r}}{r^{4}} S(r) d A
$$

The schematic of the SBES footprint is shown in figure 6.3.

To numerically compute the returned SBES envelope, the following equations are defined in terms of the area of the ensonified area.

$$
y(t)=\int_{x_{1}(t)}^{x_{2}(t)} \sigma_{b}\left(\tan ^{-1}\left(\frac{x}{H}\right)\right) \times B\left(\tan ^{-1}\left(\frac{x}{H}\right)\right) S\left(\frac{2}{c}\left(r_{2}-r\right)\right) 2 \pi x d x
$$

for $t \leq t_{0}+T$

$$
x_{1}(t)=0
$$




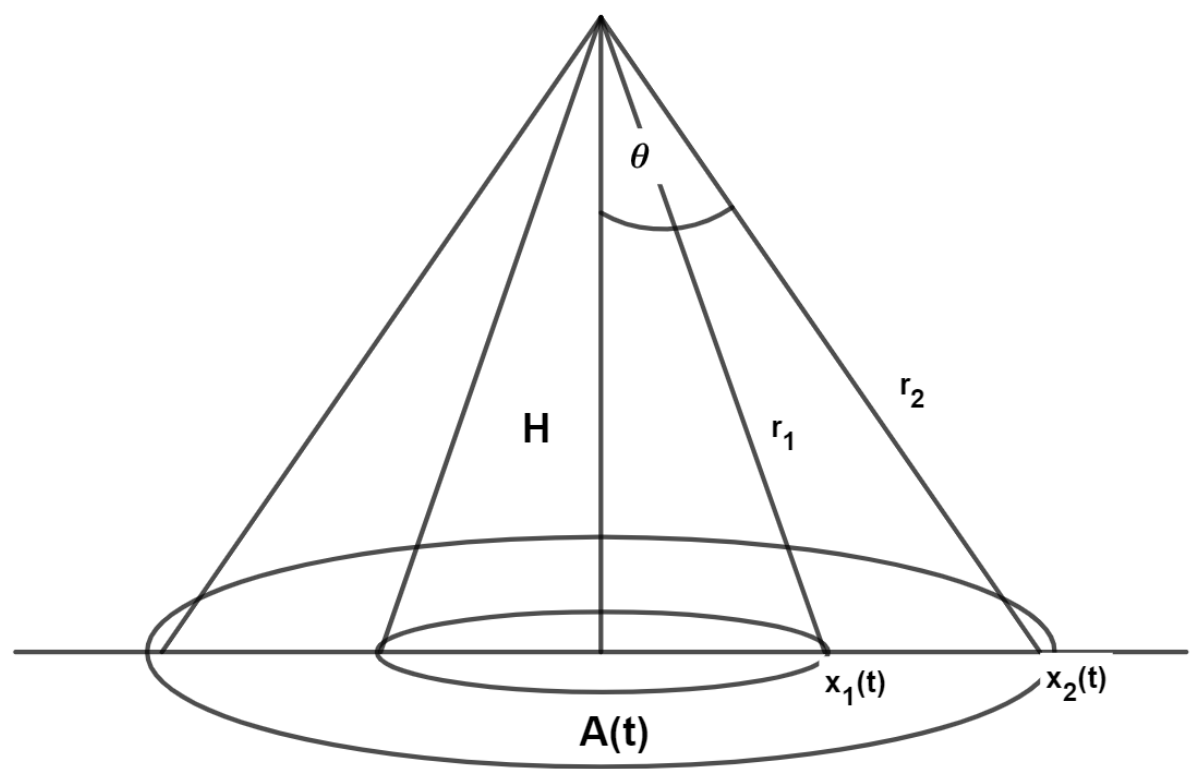

Figure 6.3: Geometry of Snellen, Siemes, and Simon Model (Adapted from (5)) 
for $t>t_{0}+T$

$$
x_{1}(t)=\sqrt{\left(\frac{c t}{2}-\frac{c T}{2}\right)^{2}-H^{2}}
$$

for $t>t_{0}$

$$
\begin{gathered}
x_{2}(t)=\sqrt{\frac{c^{2} t^{2}}{4}-H^{2}} \\
t_{0}=\frac{2 H}{c}
\end{gathered}
$$

$$
r=\sqrt{x^{2}+H^{2}}
$$

$$
r_{2}=\sqrt{x_{2}^{2}+H^{2}}
$$

The scattering parameter in eq. 6.19 is $\sigma_{b}$ which is the combination of interface and volume scattering.

$$
\sigma_{b}(\theta)=\sigma_{v}(\theta)+\sigma_{s}(\theta)
$$

\subsection{Model Validation}

In order to select a forward model, parameters and classification results were compared between the different scattering models. The accuracy of the Pinson model is validated by comparison with an exact integral solution and the physics based echo envelope model. Fifty realizations of Gaussian random surfaces were generated and passed to each model as input for the seabed. 
Return echo envelopes were simulated for six surface types: smooth, slightly rough, moderately rough, considerably rough, very rough, and extremely rough which are defined in table 6.1. Additionally, three source frequencies were simulated: $1.5 \mathrm{kHz}$ (low), $5 \mathrm{kHz}$ (moderate), and $10 \mathrm{kHz}$ (high), all using a bandwidth of $1 \mathrm{kHz}$.

The exact integral solution provides the most accurate solution because makes no physics based approximations for interface scattering and will be used as the ground truth.

A total of nine simulated test cases were analyzed the three models. To fully compare these models, different seabed types and different source frequencies were simulated to determine the validity of these models at a variety frequencies. Below is a table that lists the parameters used:

\begin{tabular}{ccc}
\hline \multicolumn{3}{c}{ Gaussian Interface Parameterization } \\
\hline Surface Type & RMS height $(\mathrm{cm})$ & Correlation Length $(\mathrm{m})$ \\
Smooth & 1.25 & 15.0 \\
Slightly Rough & 10.0 & 5.0 \\
Moderately Rough & 5.0 & 0.75 \\
Considerably Rough & 5.0 & 1.0 \\
Very Rough & 7.5 & 0.5 \\
Extremely Rough & 2.5 & 0.05 \\
\hline
\end{tabular}

Table 6.1: RMS Height and Correlation Length for Simulated Rough Interfaces 


\subsection{1 $10 \mathrm{kHz}$ Source}

A $10 \mathrm{kHz}$ source with a bandwidth of $1 \mathrm{kHz}$ was simulated over the six rough surfaces defined in table 6.1. This signal was selected, since the physics based acoustic scattering models are typically valid at high frequencies (2). Overlaid model comparison of simulated echo envelopes are shown in fig. 6.4 and fig. 6.5 .

\subsection{2 $5 \mathrm{kHz}$ Source}

The second source frequency was $5 \mathrm{kHz}$ again with a bandwidth of $1 \mathrm{kHz}$. This frequency is utilized in both side scan sonars and single beam echosounders, which are the acoustic systems of interest in this research. Model comparison of simulated echo envelopes are shown in fig. 6.6 and fig. 6.7

\subsection{3 $\quad 1.5 \mathrm{kHz}$ Source}

Lastly a source operating at $1.5 \mathrm{kHz}$ was simulated to determine if the Pinson model had a lower bound on valid frequency ranges. Model comparison of simulated echo envelopes are shown in fig. 6.8 and fig. 6.9 


\section{$10 \mathrm{kHz}$ Model Comparison}
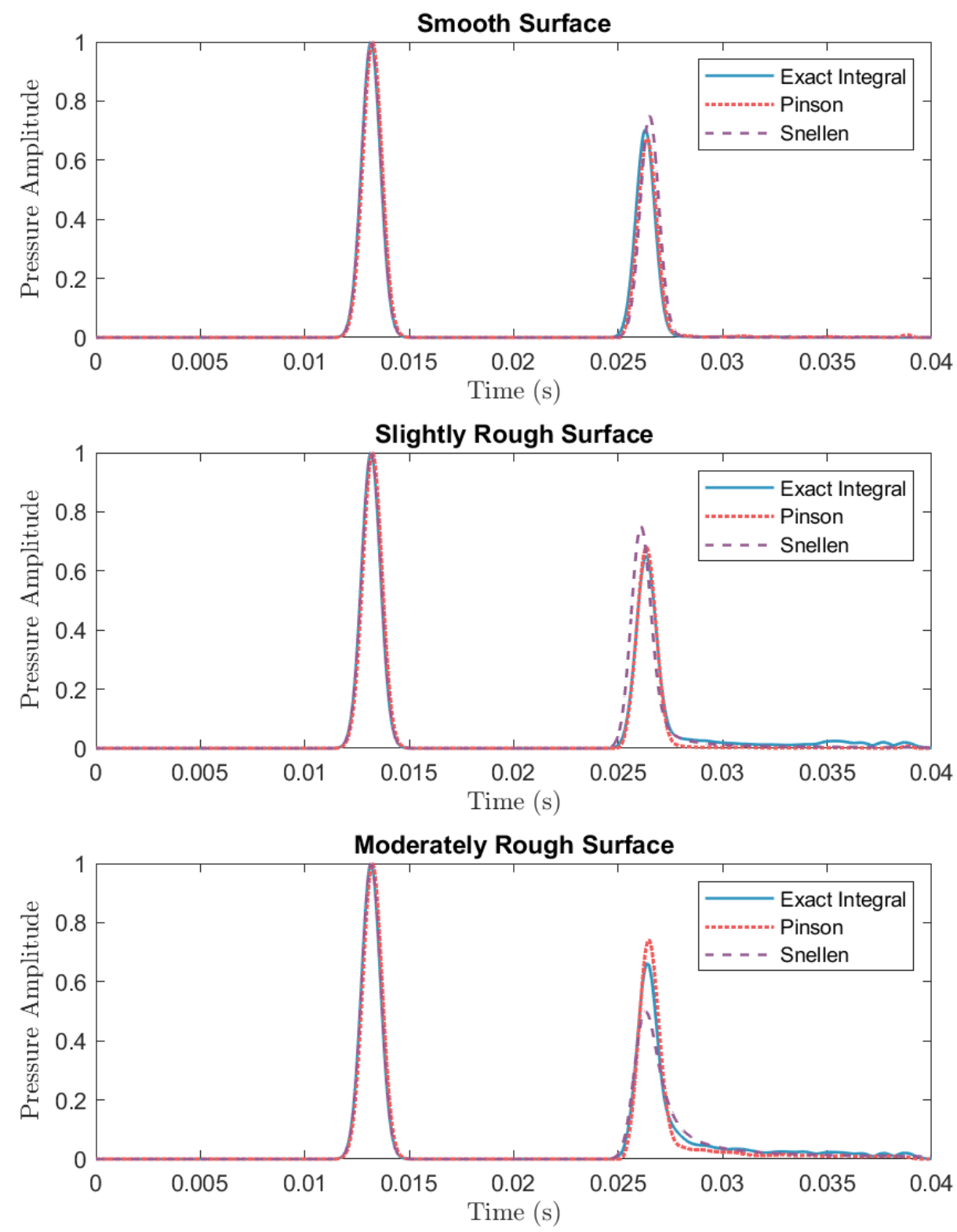

Figure 6.4: Pinson model compared to exact integral equation and Snellen envelope model for different smooth surface types at $10 \mathrm{kHz}$ 


\section{$10 \mathrm{kHz}$ Model Comparison}
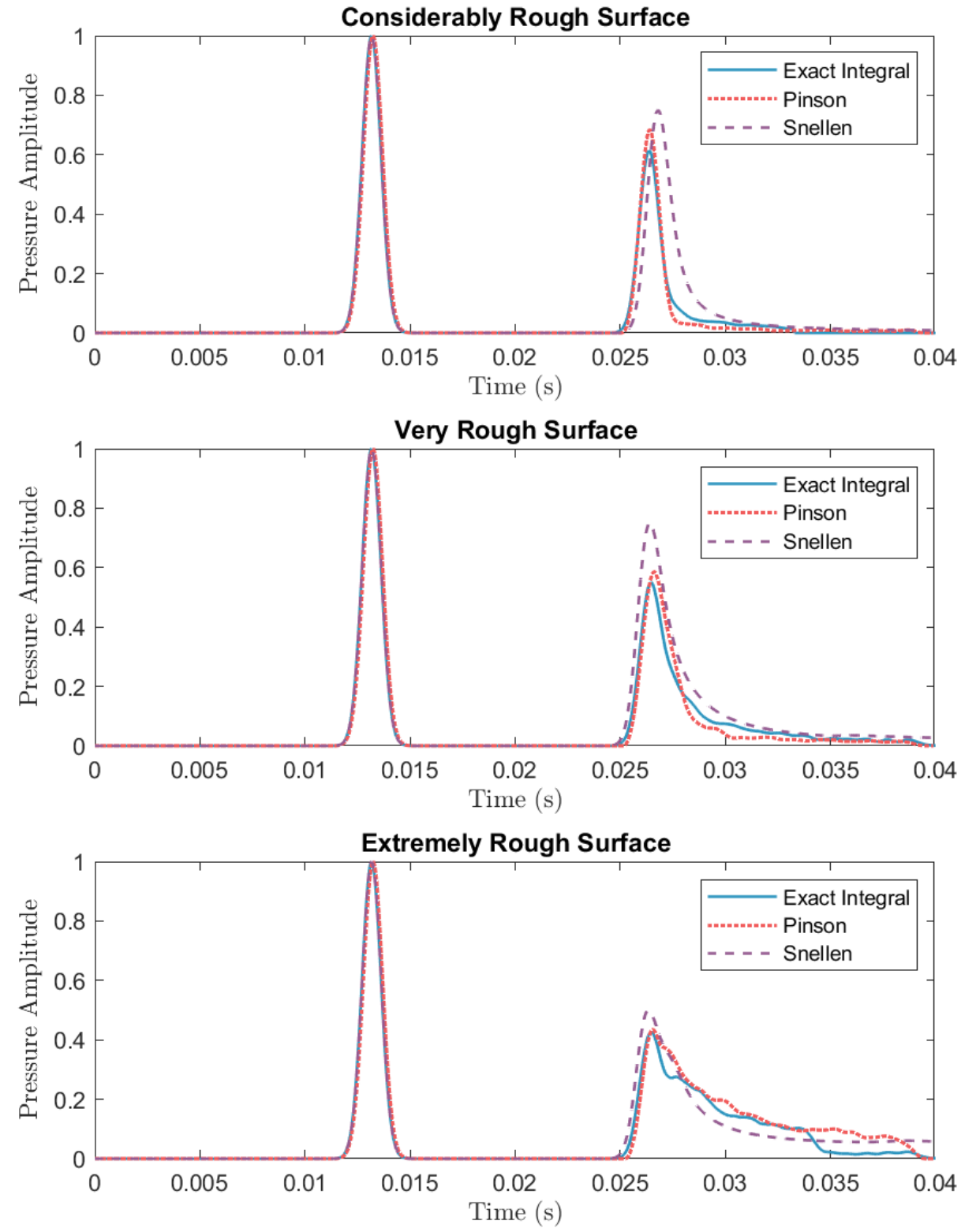

Figure 6.5: Pinson model compared to exact integral equation and Snellen envelope model for different rough surface types at $10 \mathrm{kHz}$ 


\section{$5 \mathrm{kHz}$ Model Comparison}
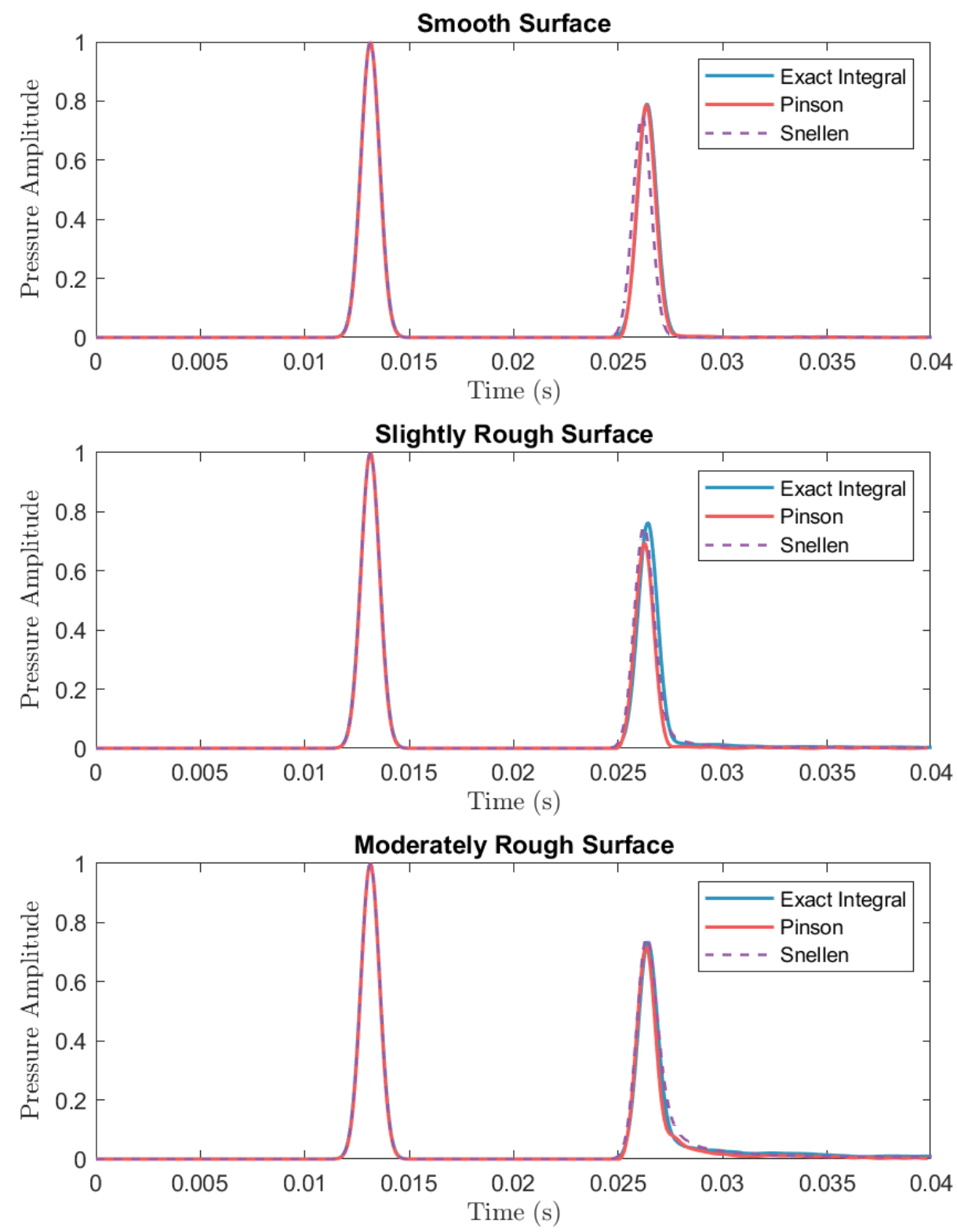

Figure 6.6: Pinson model compared to exact integral equation and Snellen envelope model for different smooth surface types at $5 \mathrm{kHz}$ 


\section{$5 \mathrm{kHz}$ Model Comparison}
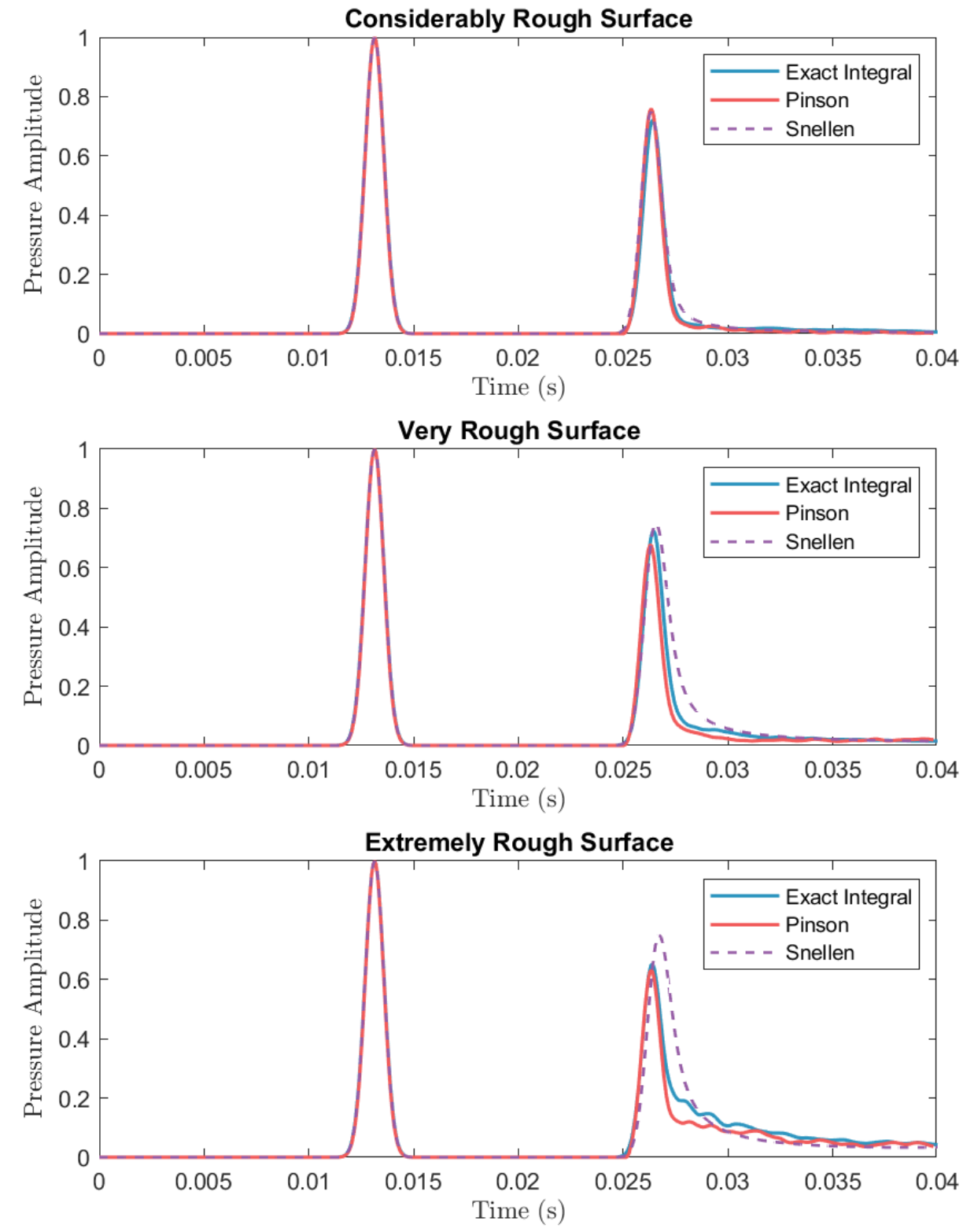

Figure 6.7: Pinson model compared to exact integral equation and Snellen envelope model for different rough surface types at $5 \mathrm{kHz}$ 


\section{$1.5 \mathrm{kHz}$ Model Comparison}
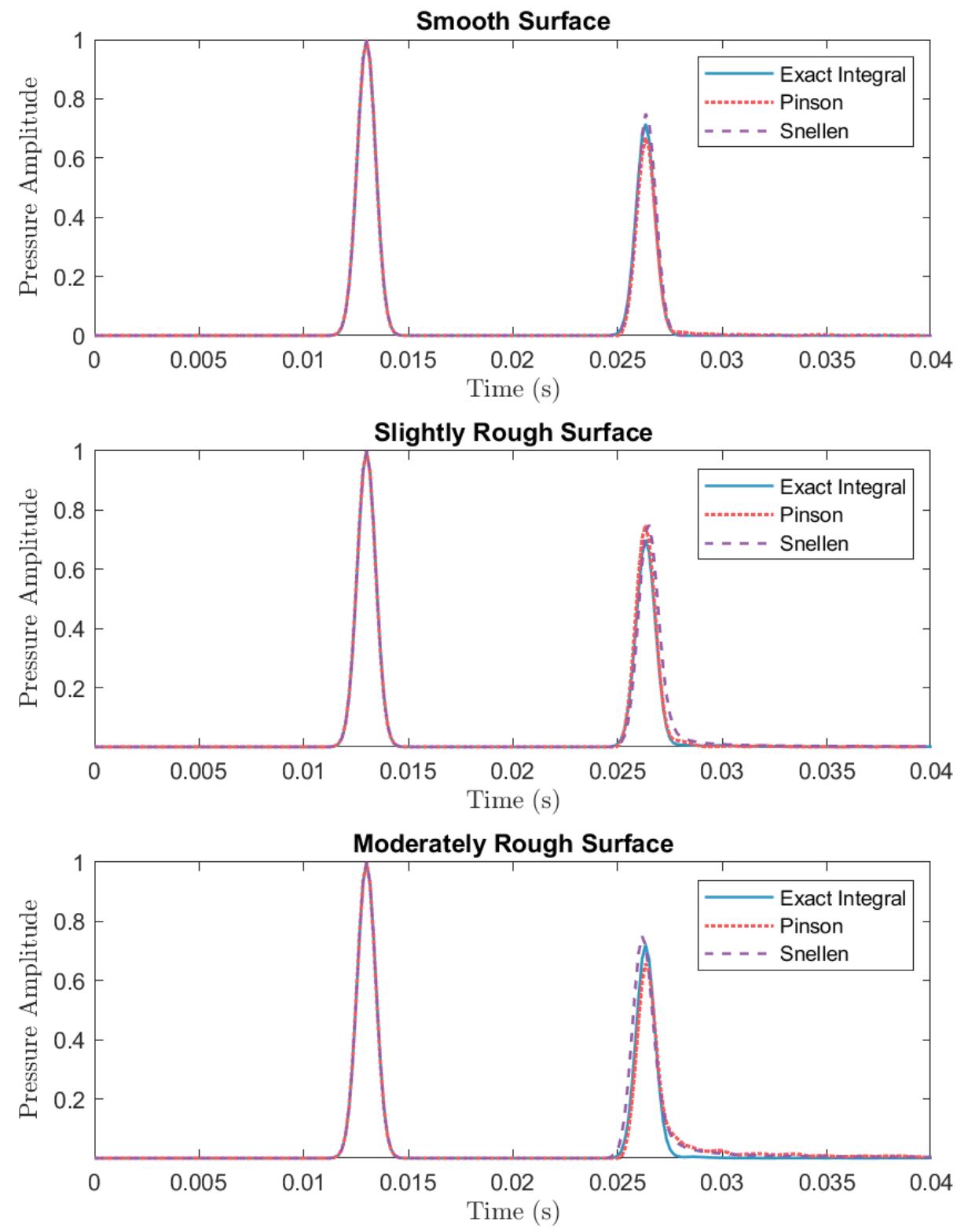

Figure 6.8: Pinson model compared to exact integral equation and Snellen envelope model for different smooth surface types at $1.5 \mathrm{kHz}$ 


\section{$1.5 \mathrm{kHz}$ Model Comparison}
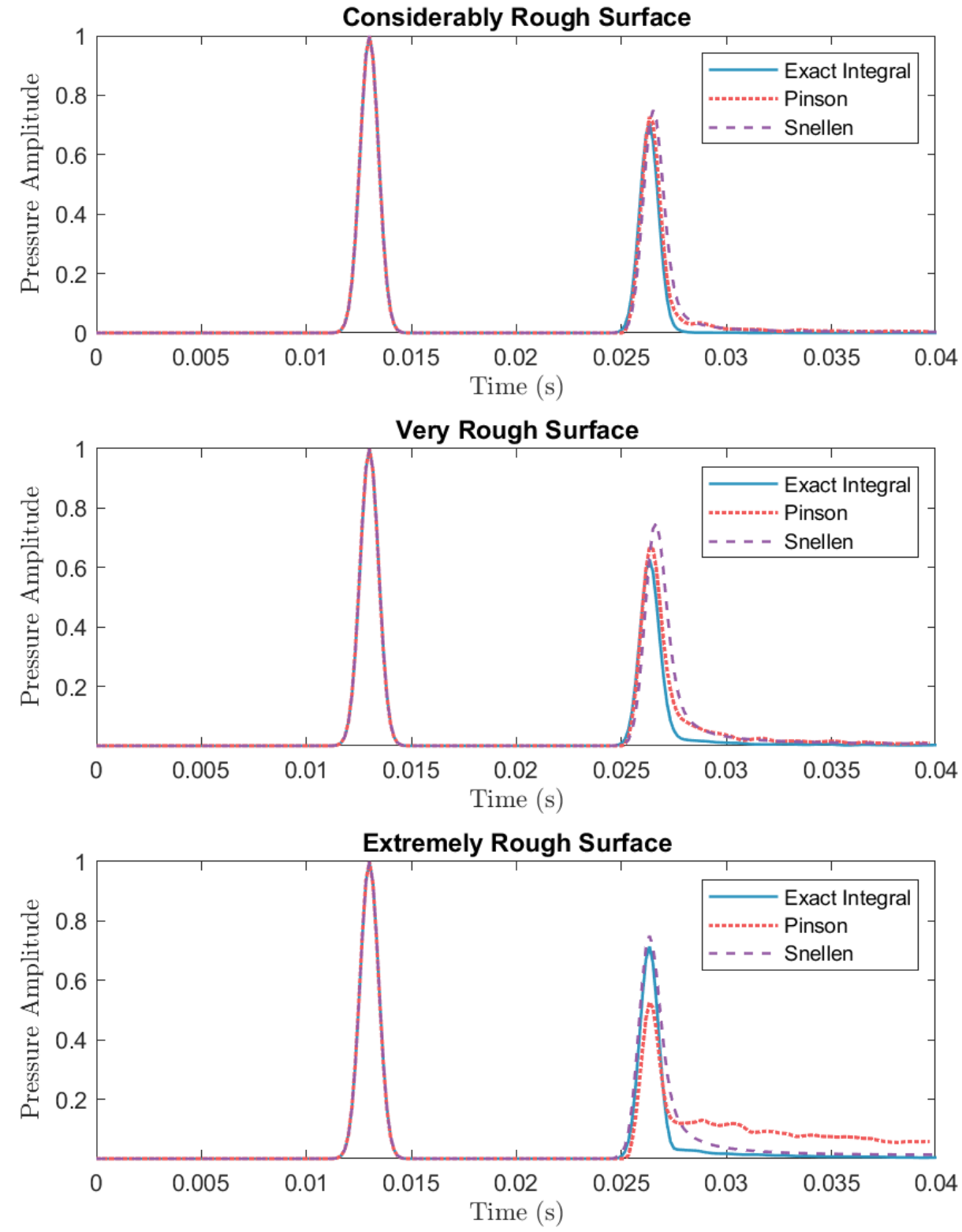

Figure 6.9: Pinson model compared to exact integral equation and Snellen envelope model for different rough surface types at $1.5 \mathrm{kHz}$ 


\subsection{Summary and Discussion of Modeling Results}

Figures 6.4 to 6.9 display the results for all three models over six seabed types and three frequencies. When the interface is smooth, all models at all frequencies return very similar envelopes. However, as the surface roughness increases the Pinson and Snellen models diverge from the exact solution.

In fig. 6.9 the extremely rough surface results for the Pinson model do not match the returned results from the exact or the approximated envelope models. Upon investigation this result can be explained by the Kirchhoff approximation. The constraint in eq. 4.1 is not satisfied in this test case. This equation can be rewritten as

$$
2 k R_{c} \sin ^{3} \theta_{g} \geq 1
$$

For a Gaussian roughness spectrum, which was used in these test cases,

$$
R_{c}=\frac{L^{2}}{\sqrt{12} h}\left(1+\frac{2 h^{2}}{L}\right)^{\frac{3}{2}}
$$

This 'Kirchhoff parameter' defines the valid region of approximation (30). For the extremely rough surface, $R_{c} \approx .03$ which means $0.06 k \sin ^{3} \theta_{g} \geq 1$. At normal incidence, this inequality relative to wavelength becomes $\frac{12 \pi}{100} \geq \lambda$. This inequality is not satisfied at $1.5 \mathrm{kHz}$, explaining the results shown in fig. 6.9 .

The amplitude and shape of the envelope returned by the Snellen model do 
agree with the results from the exact integral and Pinson model. However, the smoothing of the envelope tail muddies the differences between rough surfaces. For example at $5 \mathrm{kHz}$, the Snellen envelope for moderately and considerably rough surfaces are almost the same, as are the results for very and extremely rough surfaces. The over simplification and lack of randomness and the possibility of anomalies in the seafloor make this model have limited applications.

The Snellen model is computationally cheap but it makes large generalizations about the seafloor, which result in the over smoothed signal envelopes. Despite the Kirchhoff approximation limiting the range of frequencies below $4 \mathrm{kHz}$ the Pinson model returns results that are more exact than the Snellen envelope model. The Pinson model can also be applied to non-pressure release surfaces, 3D geometries and multiple sources. It is a "Goldie Locks" model choice since it has the best trade off between computational complexity and accuracy. 


\section{Chapter 7}

\section{Inversion and Classification}

The forward model has been selected -the Pinson model-- and validated so that it can be applied to seabed classification. However, an inverse method is another requirement to determine the parameters that define the seabed types. Most inverse methods involve two stages. First, define a cost function (cost and objective function will be used interchangeably) $C$ that quantifies the difference between the measured and simulated data (31). The second stage is to seek the values $\hat{\mathbf{m}}$ of the parameters in $\mathbf{m}$ that minimize the cost function.

\subsection{Objective Function: Huber Loss}

The objective (cost) function determines the difference between the real and simulated data while incorporating constraints (32). There are a variety of objective functions for parameter estimation, not limited to but including residuals, unweighted least squares, weighted least squares, and multiple linear regression (31). The most commonly used methods in linear programming are residuals and unweighted least squares. Residuals are more commonly referred to as the mean absolute error (MAE) defined in eq. 7.1, where $f$ is the forward model.

$$
C(\mathbf{d}, f(\hat{\mathbf{m}}))=|\mathbf{d}-f(\hat{\mathbf{m}})|
$$


Unweighted least squares are simply the mean squared error (MSE) defined in eq. 7.2

$$
C(\mathbf{d}, f(\hat{\mathbf{m}}))=\left(\frac{\sum_{n=1}^{N} d_{n}-f(\hat{\mathbf{m}})_{n}}{N}\right)^{2}
$$

The objective function used in the inversion method determines the parameters space that will be used and how it is updated, so the determining the best function for the specific problem is crucial (31).

In this research, Huber loss, defined in eq. 7.3 is the objective function to be minimized,

$$
C_{\delta}(y, f(x))= \begin{cases}\frac{1}{2}(\mathbf{d}-f(\hat{\mathbf{m}}))^{2} & \text { for }|\mathbf{d}-f(\hat{\mathbf{m}})| \leq \delta \\ \delta|\mathbf{d}-f(\hat{\mathbf{m}})|-\frac{1}{2} \delta^{2} & \text { otherwise. }\end{cases}
$$

Huber loss was selected as the objective function for scattering parameter estimation because it is robust against asymmetric departures from normality (33). This is a desirable property in underwater acoustics, since small aberrations in the seafloor can cause outliers in the data, especially when the ensemble size is small. The envelope of the signal is also heavy-tailed or skewed, which causes mean squared error to become sub-optimal since the deviations from the empirical mean are greater (34).

Huber loss is a combination of mean absolute error and mean squared error. The tunable parameter $\delta$ determines which error function will be applied to a specific point. The ability to switch between these two error functions makes the difference metric. If the error e is $>>1 \mathrm{MSE}$ gives more weight to outliers, which is undesirable because random noise and interference will 
corrupt the mean value (34). MAE returns the median of the dataset, which can also be undesirable if the data contains large sections of silence or irrelevant information. Selecting a value for $\delta$ is critical in implementing the Huber loss function because it determines what is considered an outlier in the data. A standard value for a Gaussian random process is 1.345 (33).

\subsection{Inverse Methods}

Parameter estimation problems fall into two categories: unconstrained and constrained (31). When the parameter vector $\mathbf{m}$ is free to take on any values the problem is called unconstrained optimization. Constrained optimization means that only parameter values satisfying certain criteria are permitted. However, constraints play a relatively minor role in most estimation problems, so generally, both unconstrained and constrained problems can be solved with the same inversion methods (31).

Inversion methods are iterative in nature. Starting with a given parameter set $\hat{\mathbf{m}}_{1}$, known as an initial guess, the forward model generates predictions using a sequence of $\hat{\mathbf{m}}$ values (31). Each prediction is referred to as an iteration, and the search terminates after a finite sequence of $N$ iterations and $\hat{\mathbf{m}}$ is selected as an approximation of $\mathbf{m}$. There are three conventional iterative search methods for linear problems: Newton's method, the bisection method, and Jacobi iteration. In non-linear problems, exhaustive search is the most general iterative search (31). However, all of these methods require the forward model to be evaluated at every iteration and have a significant computational 
complexity, especially in problems where more than one parameter is being estimated; these methods are slow and inefficient since a substantial number of model evaluations are required.

When objective functions are non-linear, direct search approaches are typically used (31). The most well know are the Nelder and Mead, genetic algorithms, and evolutionary strategies. A directive search strategy requires a method to generate variations of parameter vectors and decide if the newly formed parameter vector will be accepted (32). These optimization strategies all follow the same structure. Define a discrete window of values for each parameter and update the values accordingly.

Most standard direct search methods use the greedy criterion to determine if newly derived parameters should be excepted. (32). This decision rule converges quickly but can get stuck in a local minimum. Misconvergence can be avoided in most parallel search techniques: genetic algorithms, evolutionary strategies, etc. (32). Misconvergence can also be avoided by using simulated annealing (10). Simulated annealing does not require that the parameters get updated only in the direction of the minimum; instead, this condition is relaxed, and the algorithm can 'climb' out of local minima (10).

\subsection{Differential Evolution}

Seabed classification is a constrained and non-linear parameter estimation problem, and this research utilizes an evolutionary algorithm to determine the optimal parameter set (32). Evolutionary algorithms describe a subset 
of dynamic programming inspired by biological structures that minimize an objective function (35). These algorithms are based on parameter mutation that follows a simple structure:

- Create a subset of the possible parameter combinations.

- Change the parameter values by some step size, slightly and at random.

- If the objective function value is not improved return to the old parameters (35).

There are a wide variety of evolutionary algorithms to chose from when performing parameter estimation including particle swarm optimization, bees algorithm and ant colony optimization. Differential evolution was selected as the optimization strategy in this research.

\subsubsection{Motivation}

Differential evolution was designed to fulfill four requirements:

1. Utilize nonlinear and multimodal objective functions.

2. Parallelizability to reduce computational complexity.

3. Simple implementation.

4. Consistent convergence to global minimum.

Differential evolution uses a stochastic direct search method that allows nondifferentiable functions to be optimized satisfying requirement 1 . Requirement 
2 is met by the vectorization of parameter populations, which can be updated independently. Differential evolution is simple to implement because it requires little user input and is self-organizing.

\subsubsection{Algorithm}

Differential evolution is a parallel direct search method that uses a selected number of population members, NP that contain a multidimensional parameter vector $\hat{\mathbf{m}}$.

$$
\hat{\mathbf{m}}_{i, G} \quad i=1,2, \ldots, \mathrm{NP}
$$

This parameter vector is an estimate of the true parameter vector $\mathbf{m}$ discussed in Chapter 2. The subscript $G$ represents the generation of the population member. The search space is updated or mutated by the following rule. For each target vector $\hat{\mathbf{m}}_{i, G}$ a mutant vector is generated according to

$$
\hat{\mathbf{v}}_{i, G+1}=\hat{\mathbf{m}}_{r_{1}, G}+\mathrm{F} *\left(\hat{\mathbf{m}}_{r_{2}, G}-\hat{\mathbf{m}}_{r_{3}, G}\right)
$$

where $r_{1}, r_{2}$, and $r_{3}$ are random indices that are mutually different. $F$ is the step size that controls the amplification of differential variation (32). The recommended value of $F$ is .8 , but any $F \epsilon[0,2]$ can be used. An additional search space parameter is crossover. Crossover (CR) is a constant that exist in the interval $[0,1]$ that determines if a parameter value will be updated to $\hat{\mathbf{v}}_{j i, G+1}$ or remain $\hat{\mathbf{m}}_{j i, G}$ at a specific index (32)). Note $j$ is the parameter number currently being observed. For instance in a parameter vector with five 
parameters $j$ would range from 1 to 5 . The process for updating parameters is,

$$
\hat{\mathbf{v}}_{j i, G+1}= \begin{cases}\hat{\mathbf{v}}_{j i, G+1} & \text { if }(\operatorname{randb}(j) \leq \mathrm{CR}) \text { or } j=\operatorname{rnbr}(i) \\ \hat{\mathbf{m}}_{j i, G} & \text { otherwise }\end{cases}
$$

where $\operatorname{randb}(j)$ is the $j$ th evaluation of a binary random generator and $r n b r(i)$ is a randomly selected index (32). This inverse method is summed up in fig. 7.1 .

\subsection{Inversion Structure}

Three seabed types were simulated: silt, sand, and gravel using the values found in Table 4.1. The simulated measurement data was created using a linear modulated chirp at $5 \mathrm{kHz}$ with a bandwidth of $1.5 \mathrm{kHz}$. Noise was also added to the simulated measurement data to test different signal-to-noise (SNR) values: $10 \mathrm{~dB}, 20 \mathrm{~dB}$, and $40 \mathrm{~dB}$. A von-Karman rough surface was generated with 50 realizations to get an ensemble averaging and the seabed parameters $\rho, v, \gamma_{1}$, and $w_{1}$ were inverted for. The input to the Pinson $2 \mathrm{D}$ model uses $w_{1}$ not $w_{2}$, similarly $\gamma_{1}$ instead of $\gamma_{2}$. These parameters were calculated from table 4.1 using eq. 5.3 and eq. 5.4 .

The inversion combined the objective function, Huber loss, and the optimization strategy of differential evolution. Both Huber loss and differential evolution include hyperparameters that must be set prior to evaluation. For

differential evolution: $\mathrm{NP}=40, F=.75$ and $\mathrm{CR}=.9$ were selected. The 


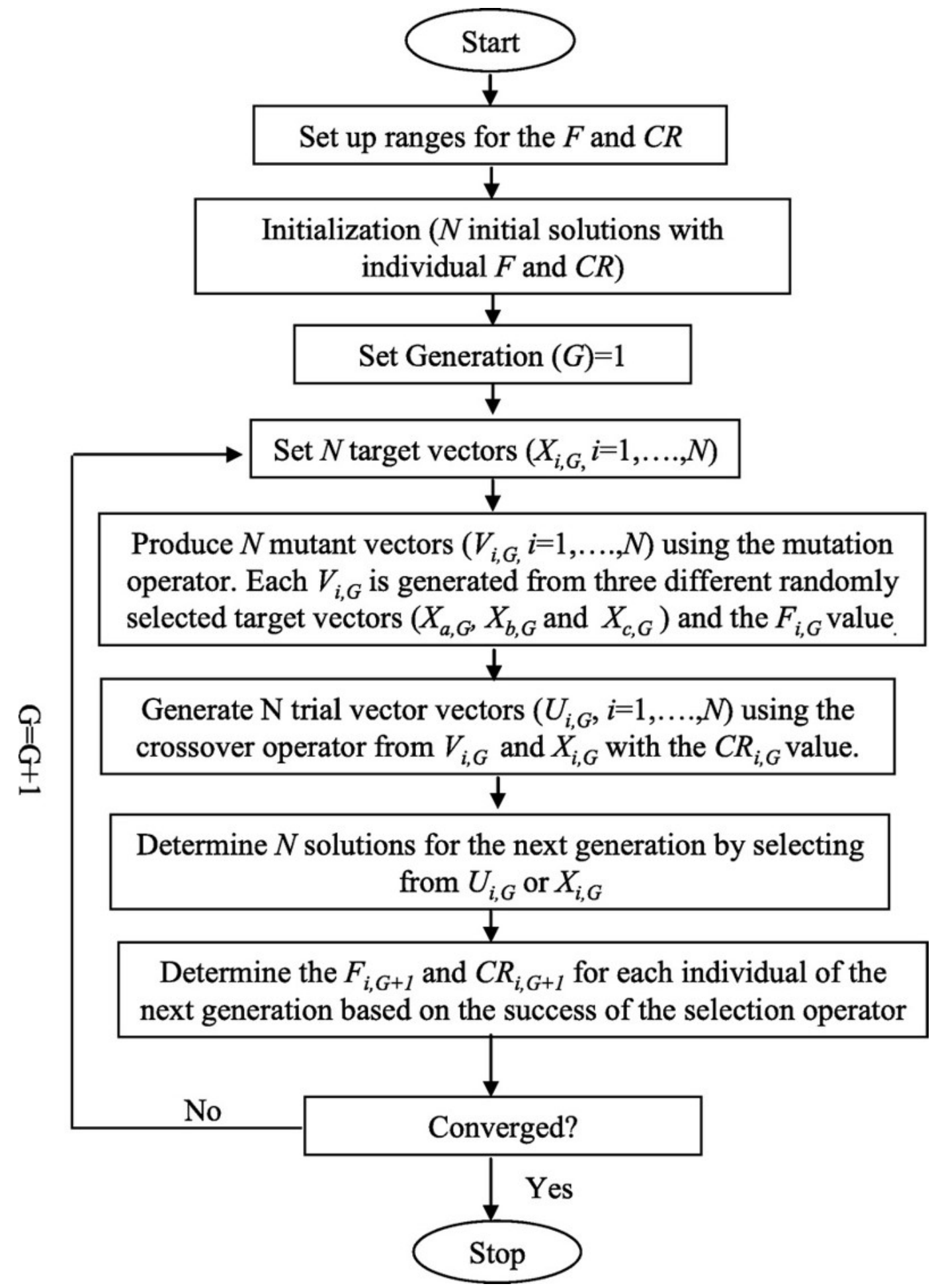

Figure 7.1: Structure of differential evolution (Source (6) ) 
Huber loss function was applied to both log and normal amplitude with each having a different $\delta$ value. For $\log$ amplitude $\delta=\operatorname{std}\left(10^{*} \log 10(\mathbf{d})\right)$ and normal amplitude $\delta=\operatorname{std}(\mathbf{d})$. Using those parameters the evaluation strategy is outlined below:

1. Produce or provide an initial parameter value vector to the differential algorithm.

2. Create predictions using these parameters for 50 rough surface realizations and average the results. This will be the simulated signal that will be compared with the simulated measurement data.

3. Find the start and endpoints of the scattered signal envelope to crop out irrelevant data. This step can also include source and simulation signal alignment, which has been ignored since this is a purely simulated study.

Evaluation of the objective function

4. For the log amplitude: determine the range of datapoints to compare the simulated signals to the simulated measurement data. The simulated measurement data will be corrupted by noise making the tail of the envelope irrelevant information. In this research 50 datapoints following the peak were used.

5. Take the absolute difference between the selected portions of simulated measurement data and simulated signal. 
6. Calculate the Huber loss for the log amplitude.

7. Calculate the Huber loss for the absolute difference between the simulated measurement data and simulated signal.

8. Add the objective function results for both log and normal amplitude.

9. Iterate until a specified time limit has been reached. In this case each experiment ran for 90 minutes resulting in approximately 4000 parameter combinations.

\subsection{Inversion Results}

The tables report the error value between the true and estimated parameters and the cost function evaluation over each parameter is displayed. The estimated signal envelope with the simulated measurement data is also shown.

\subsubsection{Sand}

The following section contains the inversion results for the seabed type sand, which has a roughness type of moderate.

\section{0dB SNR}




\begin{tabular}{cccc}
\hline \multicolumn{4}{c}{$\begin{array}{c}\text { Seabed Type: Sand } \\
\text { SNR: 40dB }\end{array}$} \\
\hline Parameters & True Value & Estimated Value & Percent Error \\
\hline$\rho$ & 1.81 & 1.8 & 0.5525 \\
$v$ & 1.18 & 1.18 & 0.0 \\
$\gamma_{1}$ & 1.95 & 1.84 & 5.6410 \\
$w_{1}$ & 0.00225 & 0.002 & 11.11 \\
\hline
\end{tabular}

Table 7.1: Inversion Results For Sand at High SNR
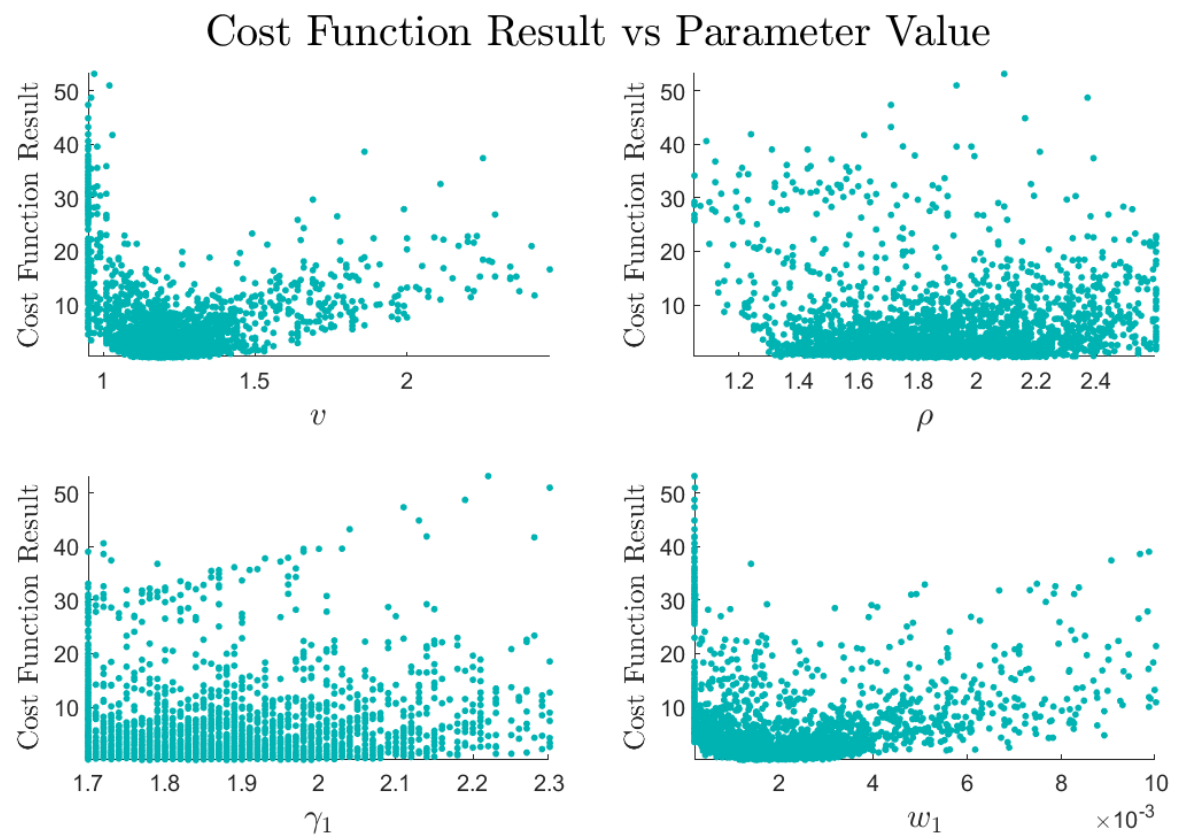

Figure 7.2: Cost function evaluation for sandy surface parameters at High SNR 


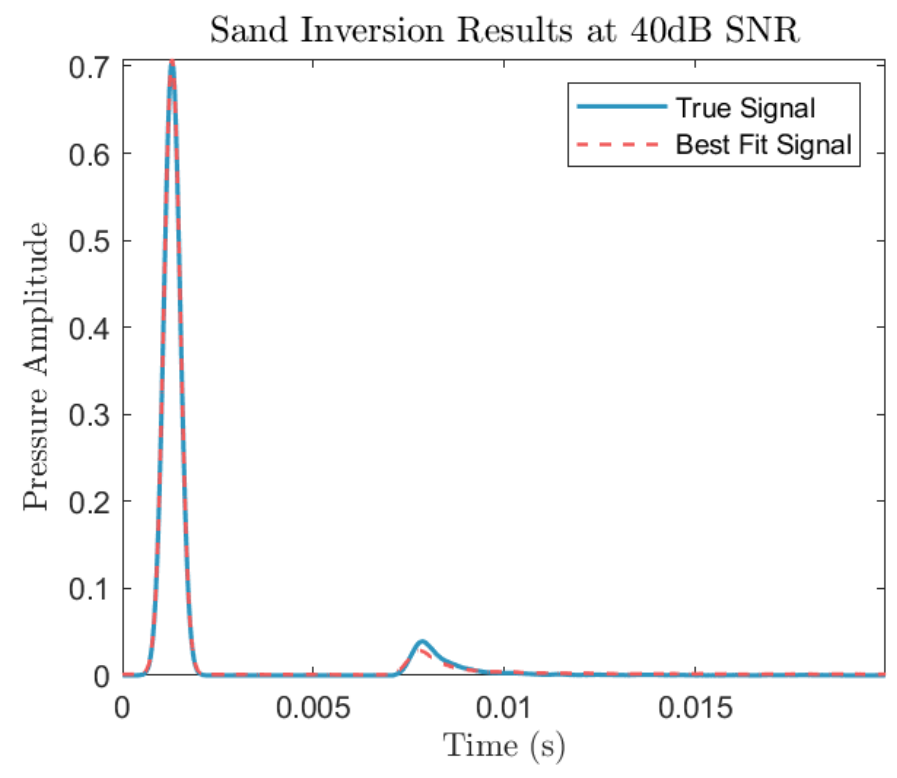

Figure 7.3: Real and estimated signal for sandy surface at high SNR

\section{0dB SNR}

\begin{tabular}{cccc}
\hline \multicolumn{4}{c}{$\begin{array}{c}\text { Seabed Type: Sand } \\
\text { SNR: 20dB }\end{array}$} \\
\hline Parameters & True Value & Estimated Value & Percent Error \\
\hline$\rho$ & 1.81 & 1.70 & 6.0773 \\
$v$ & 1.18 & 1.26 & 6.7797 \\
$\gamma_{1}$ & 1.95 & 1.97 & 1.0256 \\
$w_{1}$ & 0.00225 & 0.00220 & 3.5556 \\
\hline
\end{tabular}

Table 7.2: Inversion Results For Sand at 20dB SNR 


\section{Cost Function Result vs Parameter Value}
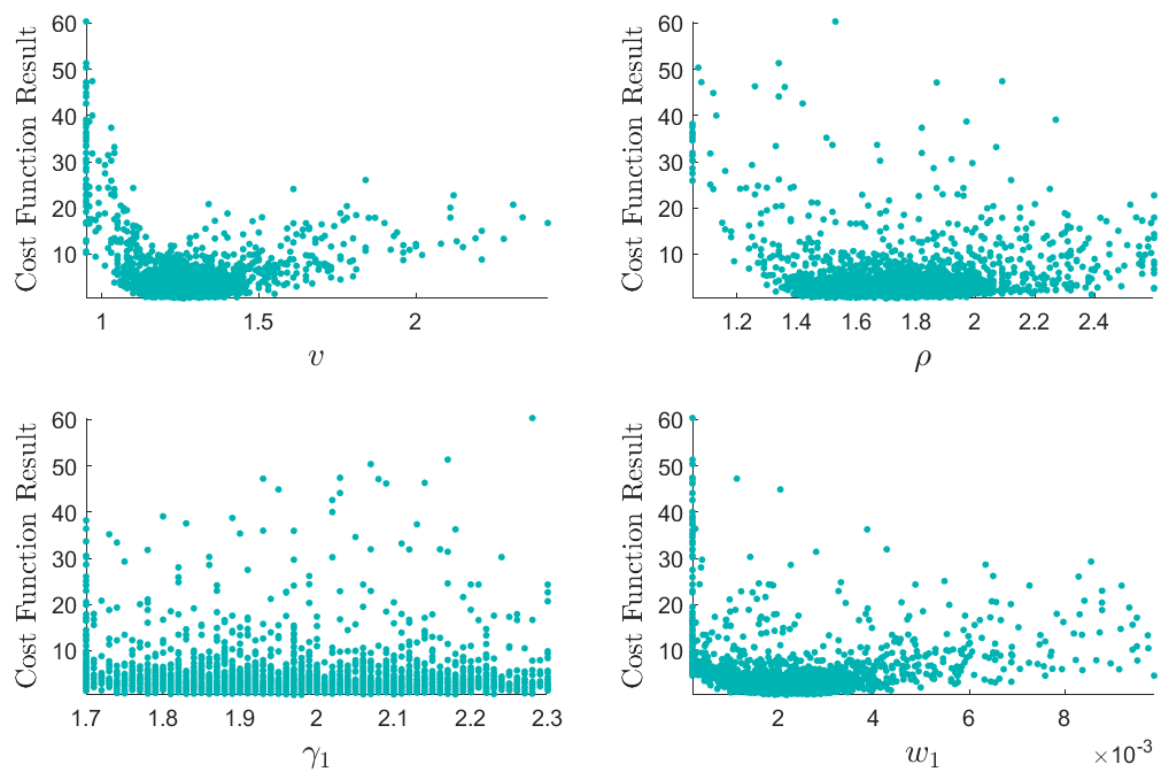

Figure 7.4: Cost function evaluation for sandy surface parameters at $20 \mathrm{~dB}$ SNR

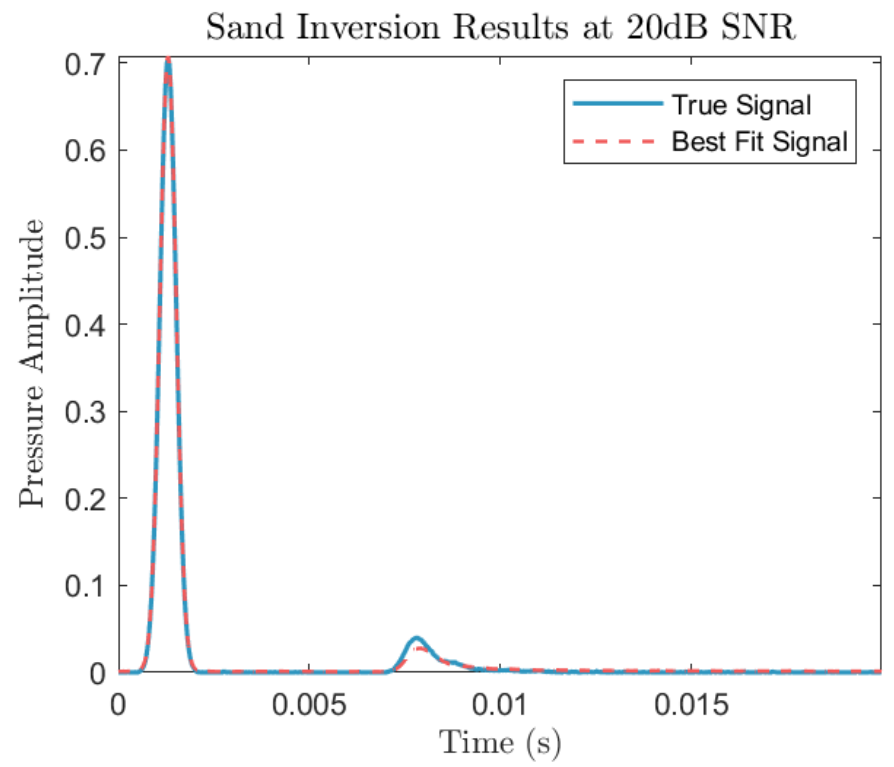

Figure 7.5: Real and estimated signal for sandy surface at 20dB SNR 


\section{0dB SNR}

\begin{tabular}{cccc}
\hline \multicolumn{4}{c}{$\begin{array}{c}\text { Seabed Type: Sand } \\
\text { SNR: 10dB }\end{array}$} \\
\hline Parameters & True Value & Estimated Value & Percent Error \\
\hline$\rho$ & 1.81 & 1.56 & 13.812 \\
$v$ & 1.18 & 1.54 & 30.51 \\
$\gamma_{1}$ & 1.95 & 2.3 & 17.9487 \\
$w_{1}$ & 0.00225 & 0.004 & 76.8889 \\
\hline
\end{tabular}

Table 7.3: Inversion Results For Sand at 10dB SNR
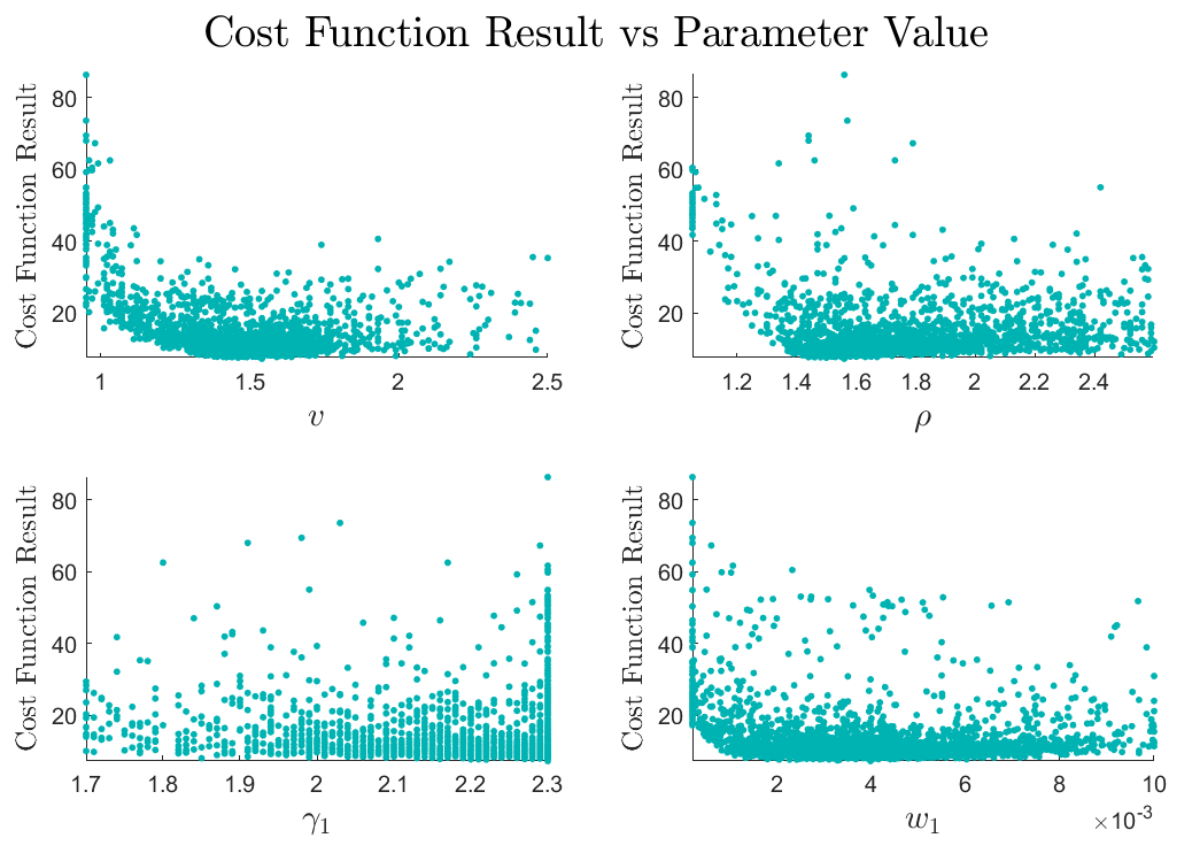

Figure 7.6: Cost function evaluation for sandy surface parameters at $10 \mathrm{~dB}$ snr 


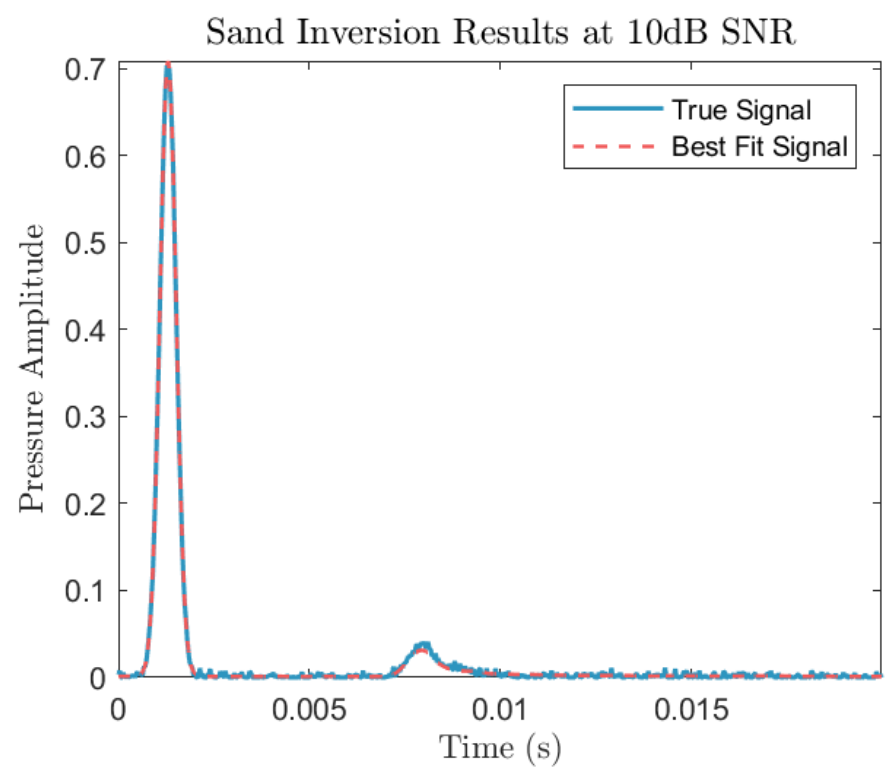

Figure 7.7: Real and estimated signal for sandy surface at 10dB SNR

\subsubsection{Gravel}

The following section contains the inversion results for the seabed type gravel, which has a roughness type rough.

\section{0dB SNR}




\begin{tabular}{cccc}
\hline \multicolumn{4}{c}{$\begin{array}{c}\text { Seabed Type: Gravel } \\
\text { SNR: 40dB }\end{array}$} \\
\hline Parameters & True Value & Estimated Value & Percent Error \\
\hline$\rho$ & 2.5 & 2.54 & 1.6 \\
$v$ & 2.49 & 2.26 & 9.2369 \\
$\gamma_{1}$ & 2.15 & 2.12 & 1.3953 \\
$w_{1}$ & 0.0093 & 0.0094 & 1.1828 \\
\hline
\end{tabular}

Table 7.4: Inversion Results For Gravel at High SNR

Cost Function Result vs Parameter Value
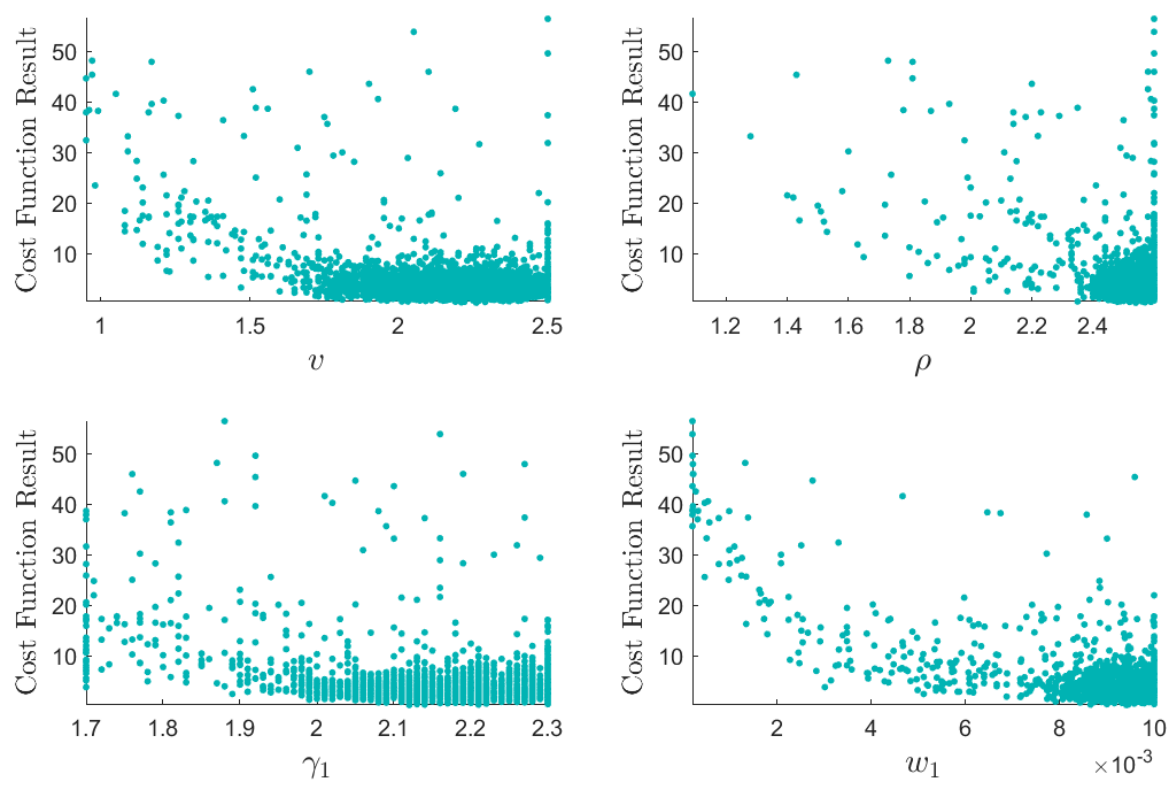

Figure 7.8: Cost function evaluation for gravel surface parameters at High SNR 


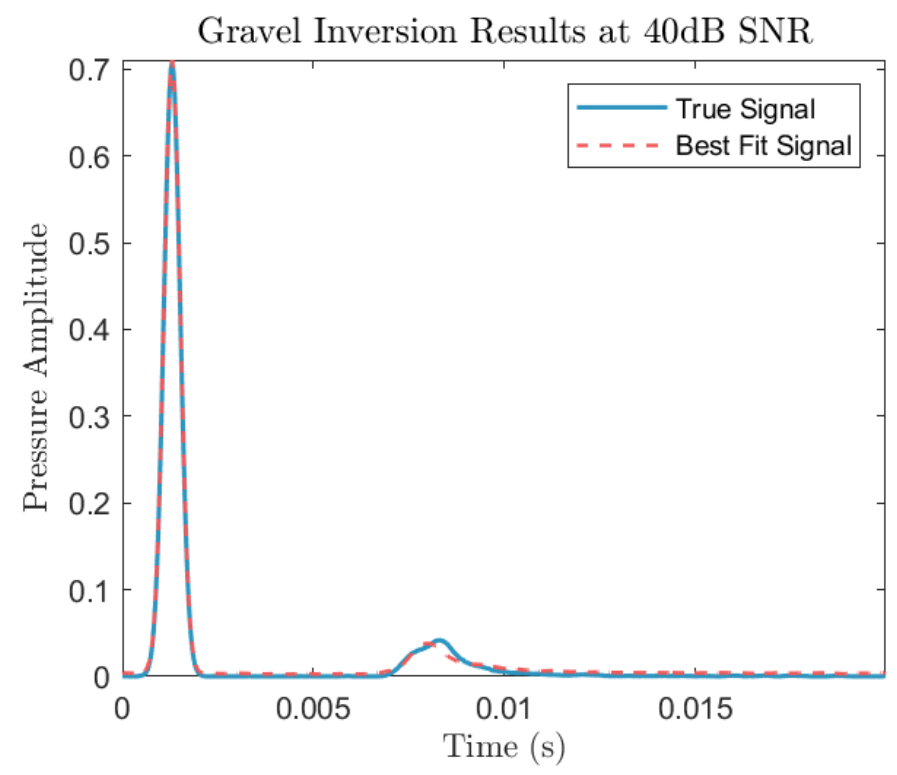

Figure 7.9: Real and estimated signal for gravel surface at High SNR

\section{0dB SNR}

\begin{tabular}{cccc}
\hline \multicolumn{4}{c}{$\begin{array}{c}\text { Seabed Type: Gravel } \\
\text { SNR: 20dB }\end{array}$} \\
\hline Parameters & True Value & Estimated Value & Percent Error \\
\hline$\rho$ & 2.5 & 2.6 & 0.40161 \\
$v$ & 2.49 & 2.5 & 4.0 \\
$\gamma_{1}$ & 2.15 & 2 & 6.9767 \\
$w_{1}$ & 0.0093 & 0.0079 & 15.4839 \\
\hline
\end{tabular}

Table 7.5: Inversion Results For Gravel at 20dB SNR 


\section{Cost Function Result vs Parameter Value}
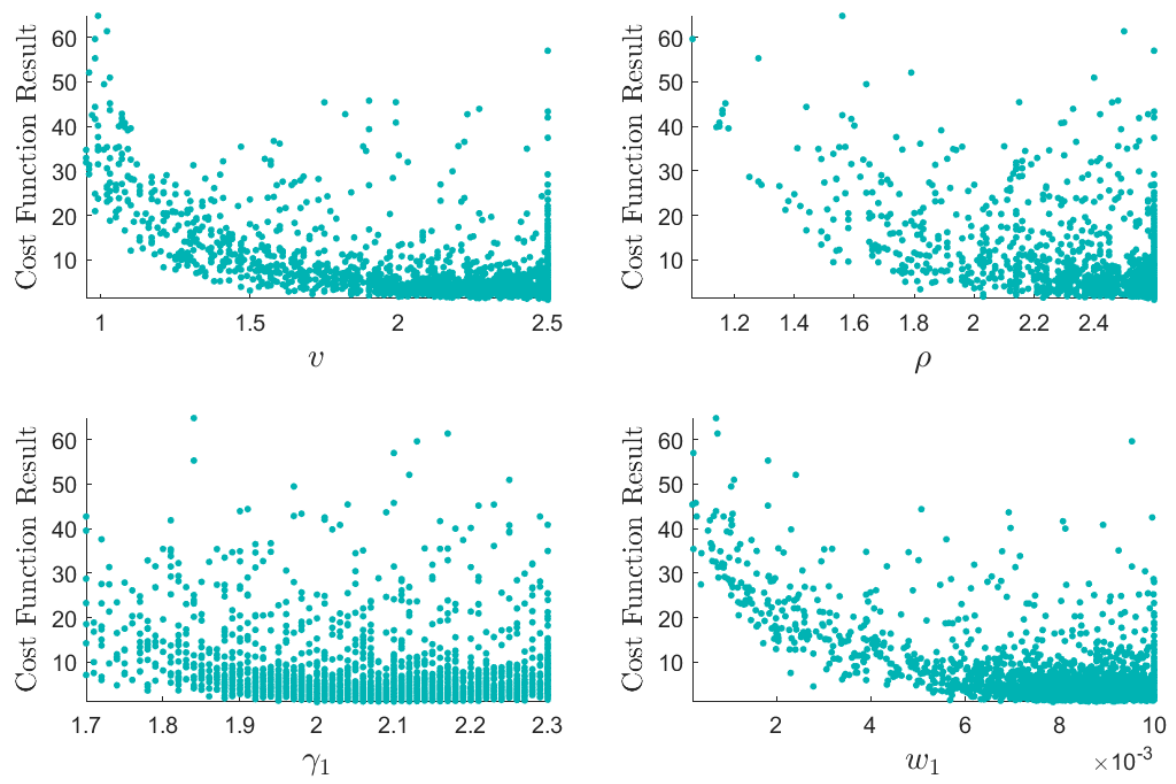

Figure 7.10: Cost function evaluation for gravel surface parameters at 20dB SNR

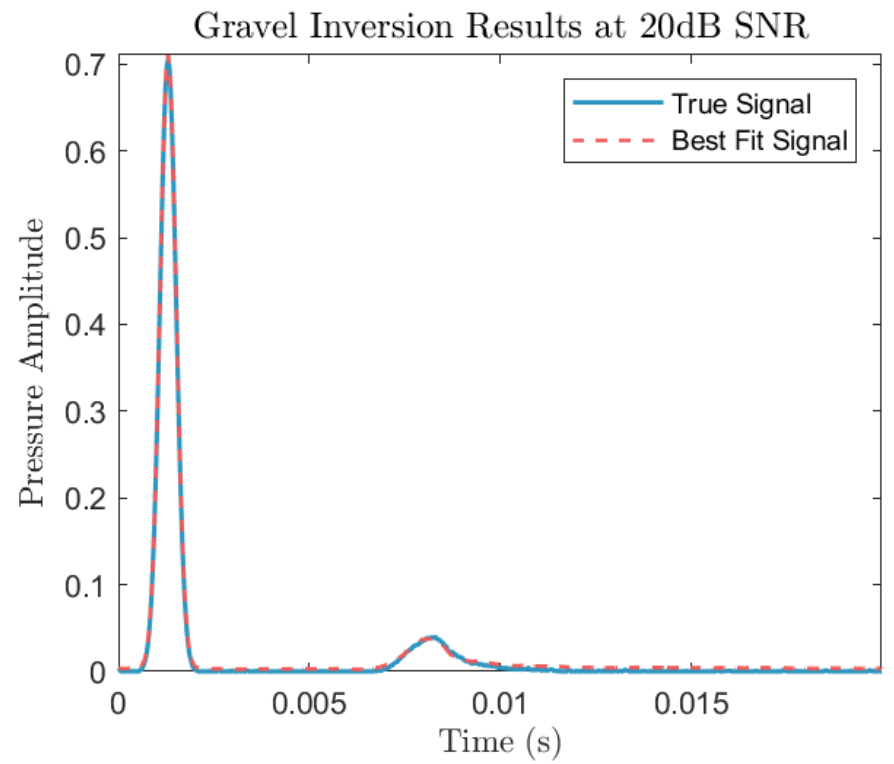

Figure 7.11: Real and estimated signal for gravel surface at 20dB SNR 


\section{0dB SNR}

\begin{tabular}{cccc}
\hline \multicolumn{4}{c}{$\begin{array}{c}\text { Seabed Type: Gravel } \\
\text { SNR: 10dB }\end{array}$} \\
\hline Parameters & True Value & Estimated Value & Percent Error \\
\hline$\rho$ & 2.5 & 2.55 & 2.0 \\
$v$ & 2.49 & 2.5 & 0.40161 \\
$\gamma_{1}$ & 2.15 & 2.3 & 6.9767 \\
$w_{1}$ & 0.0093 & 0.0096 & 3.2258 \\
\hline
\end{tabular}

Table 7.6: Inversion Results For Gravel at 10dB SNR

\section{Cost Function Result vs Parameter Value}
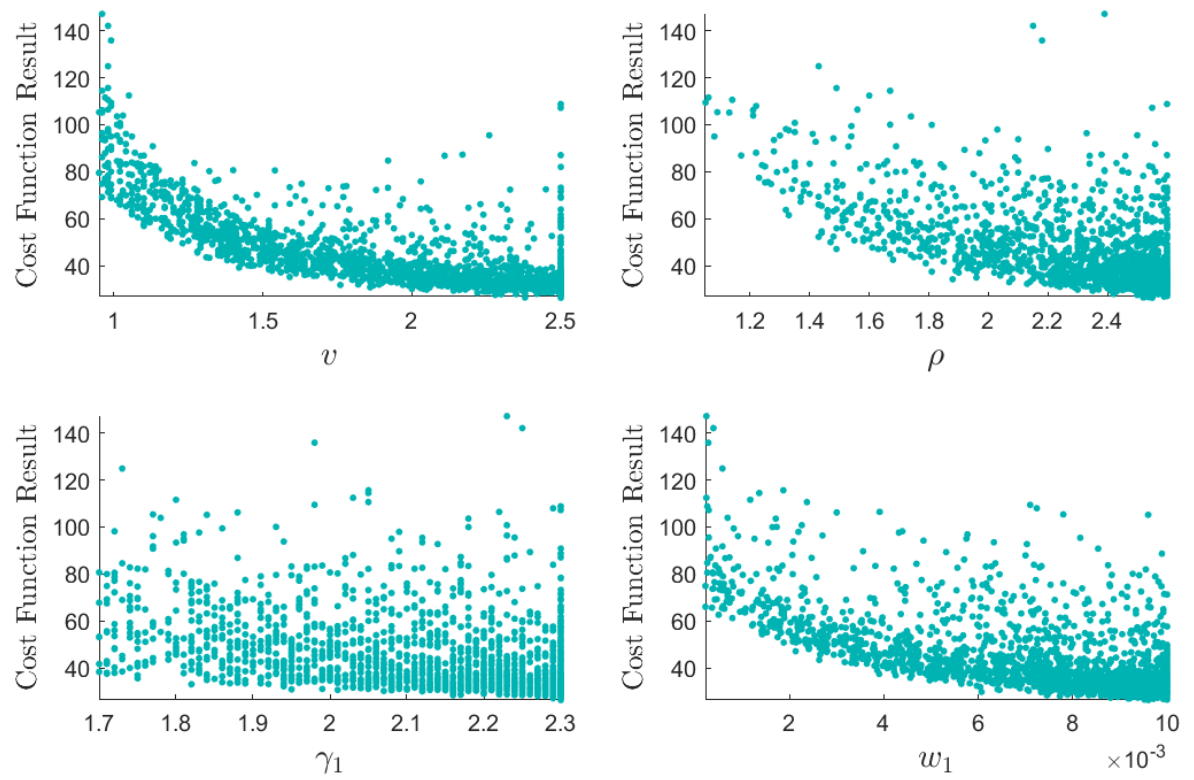

Figure 7.12: Cost function evaluation for gravel surface parameters at $10 \mathrm{~dB}$ SNR 


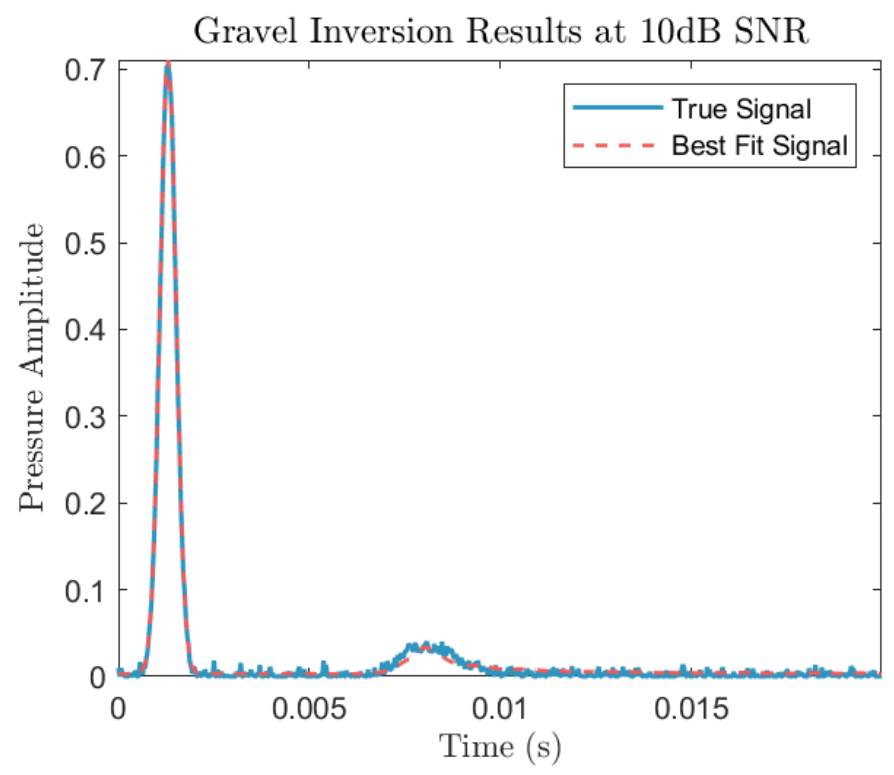

Figure 7.13: Real and estimated signal for gravel surface at 10dB SNR

\subsubsection{Silt}

The following section contains the inversion results for the seabed type silt, which has a roughness type smooth. 


\section{0dB SNR}

\begin{tabular}{cccc}
\hline \multicolumn{4}{c}{$\begin{array}{c}\text { Seabed Type: Silt } \\
\text { SNR: 40dB }\end{array}$} \\
\hline Parameters & True Value & Estimated Value & Percent Error \\
\hline$\rho$ & 1.14 & 1.14 & 0.0 \\
$v$ & 0.99 & 0.99 & 0.0 \\
$\gamma_{1}$ & 1.85 & 1.84 & 0.54054 \\
$w_{1}$ & 0.00056 & 0.00059 & 5.92 \\
\hline
\end{tabular}

Table 7.7: Inversion Results For Silt at High SNR
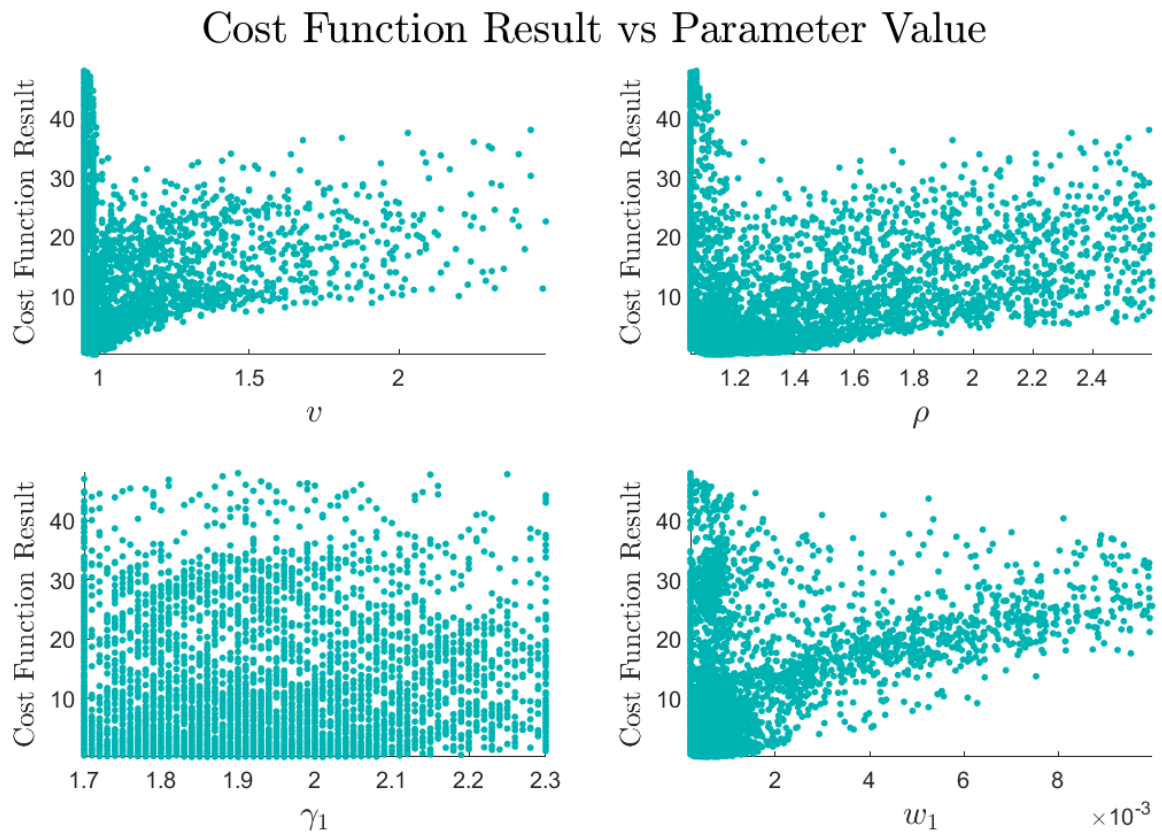

Figure 7.14: Cost function evaluation for silt surface parameters at 40dB snr 


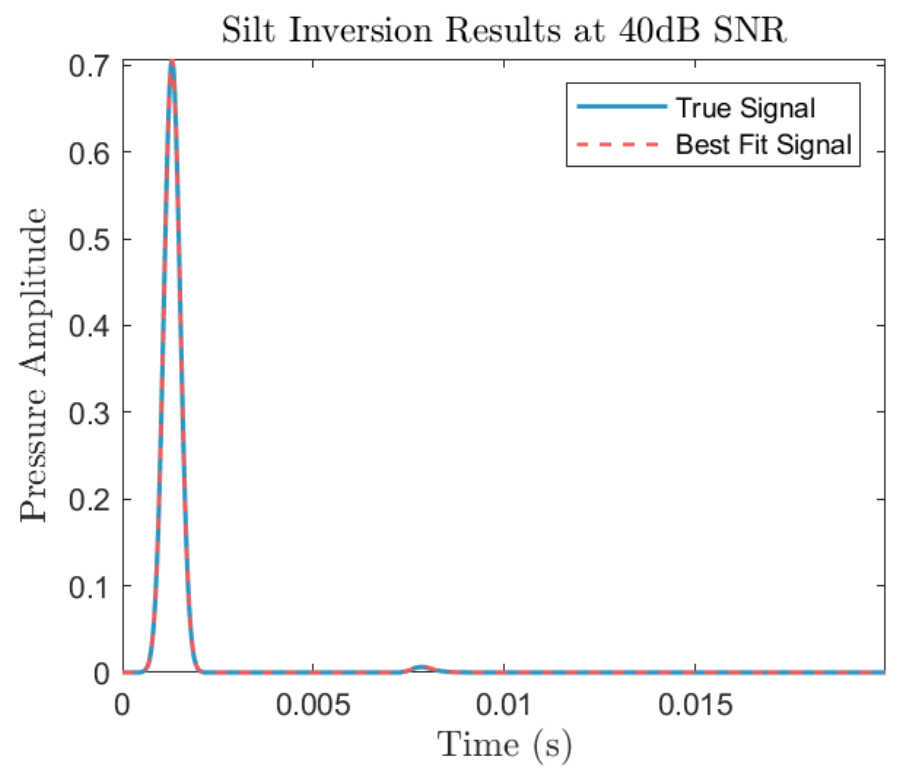

Figure 7.15: Real and estimated signal for silt surface at 40dB SNR

\section{0dB snr}

\begin{tabular}{cccc}
\hline \multicolumn{4}{c}{$\begin{array}{c}\text { Seabed Type: Silt } \\
\text { SNR: 20dB }\end{array}$} \\
\hline Parameters & True Value & Estimated Value & Percent Error \\
\hline$\rho$ & 1.14 & 1.18 & 3.5088 \\
$v$ & 0.99 & 0.98 & 1.0101 \\
$\gamma_{1}$ & 1.85 & 1.75 & 5.4054 \\
$w_{1}$ & 0.00056 & 0.00073 & 31.0592 \\
\hline
\end{tabular}

Table 7.8: Inversion Results For Silt at 20dB SNR 

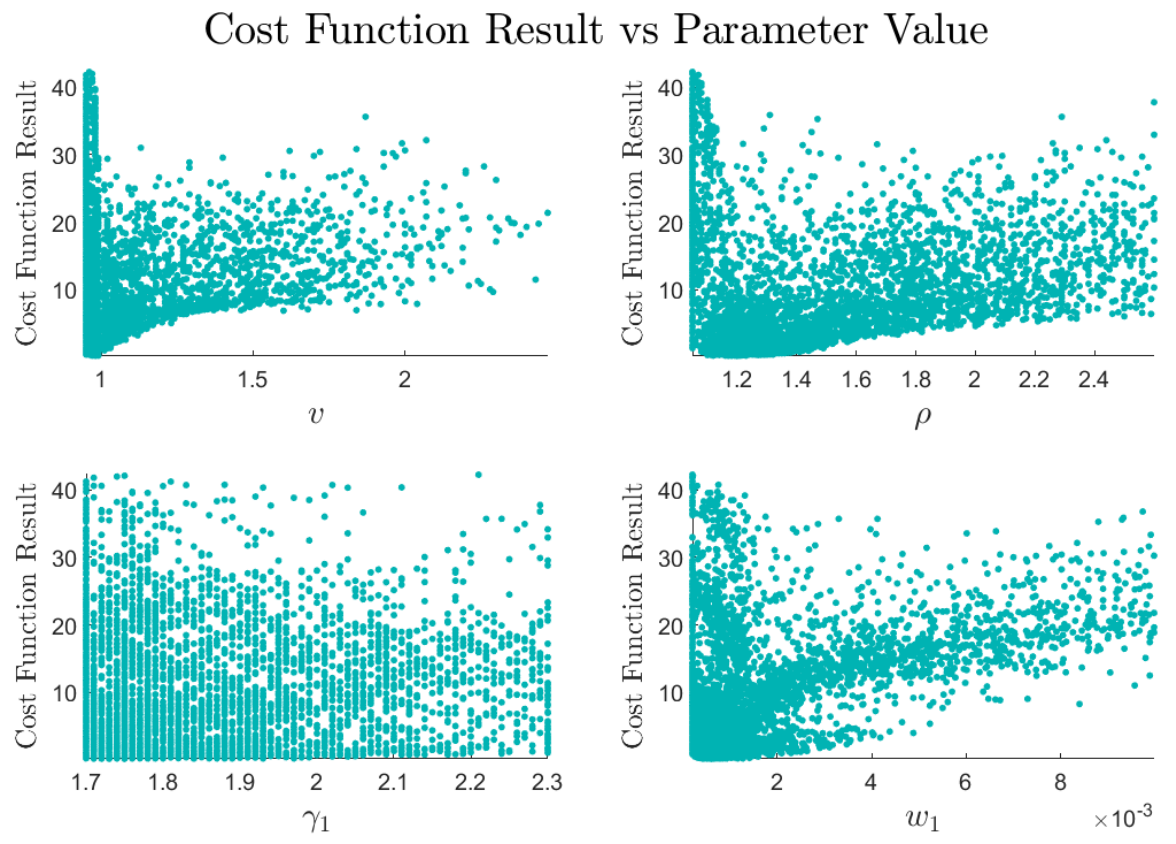

Figure 7.16: Cost function evaluation for silt surface parameters at 10dB snr

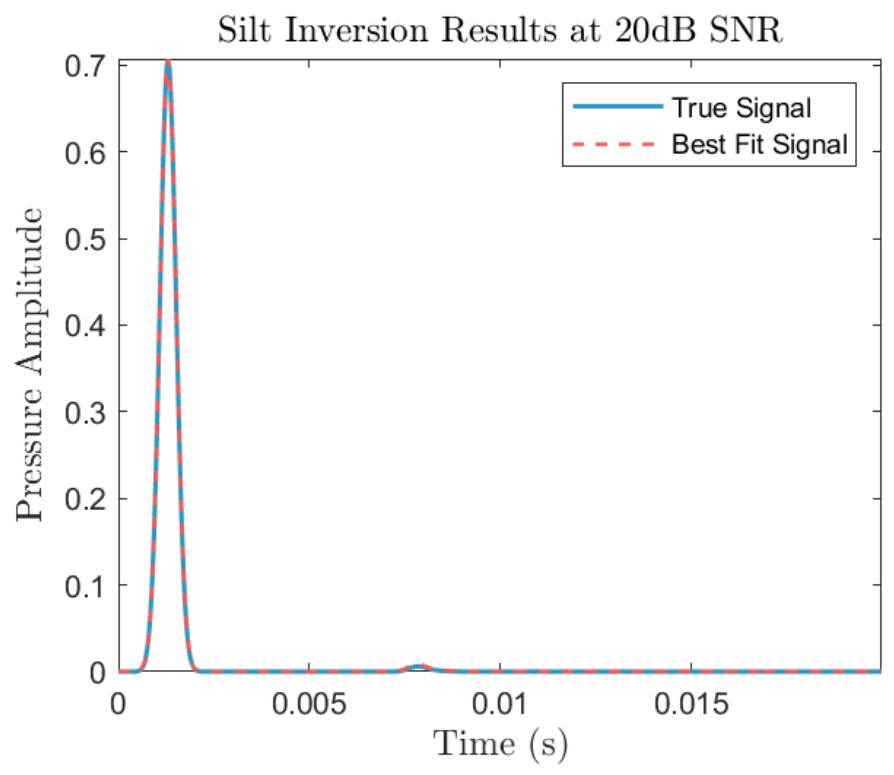

Figure 7.17: Real and estimated signal for silt surface at 20dB SNR 


\section{0dB snr}

\begin{tabular}{cccc}
\hline \multicolumn{4}{c}{$\begin{array}{c}\text { Seabed Type: Silt } \\
\text { SNR: 10dB }\end{array}$} \\
\hline Parameters & True Value & Estimated Value & Percent Error \\
\hline$\rho$ & 1.14 & 1.09 & 4.386 \\
$v$ & 0.99 & 1.03 & 4.0404 \\
$\gamma_{1}$ & 1.85 & 2.3 & 24.2343 \\
$w_{1}$ & 0.00056 & 0.00082 & 47.2172 \\
\hline
\end{tabular}

Table 7.9: Inversion Results For Silt at 10dB SNR

\section{Cost Function Result vs Parameter Value}
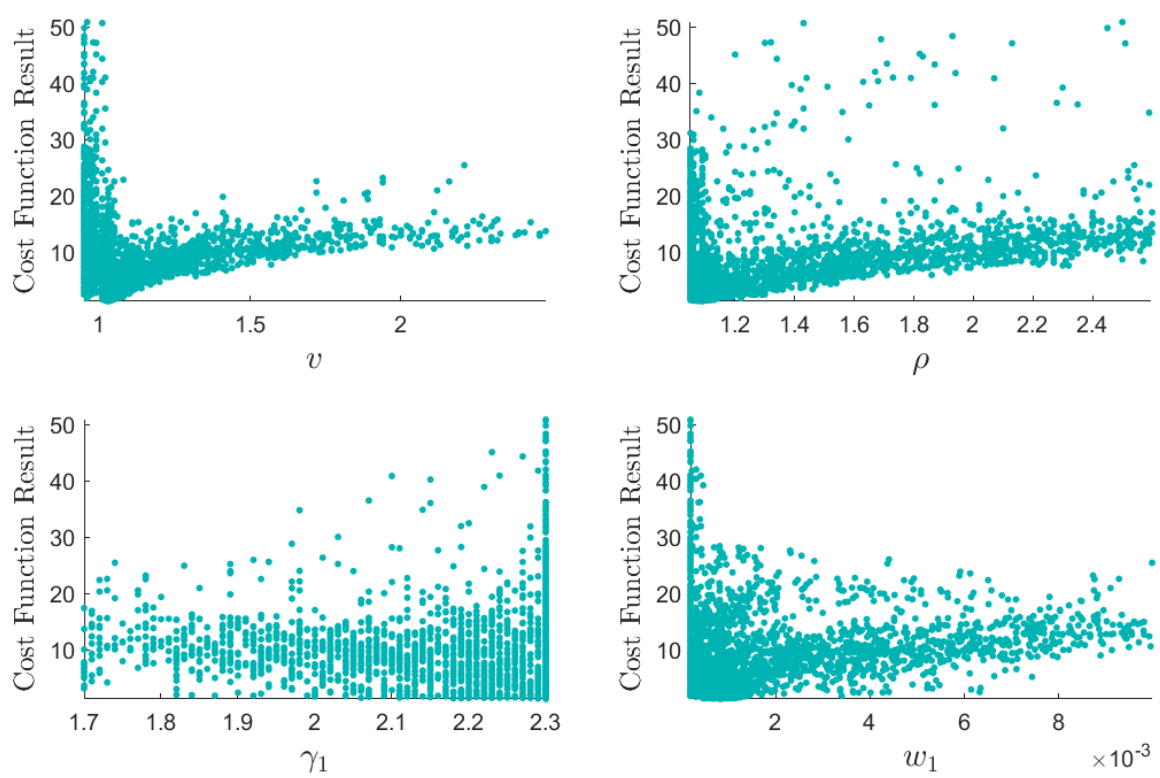

Figure 7.18: Cost function evaluation for silt surface parameters at 10dB SNR 


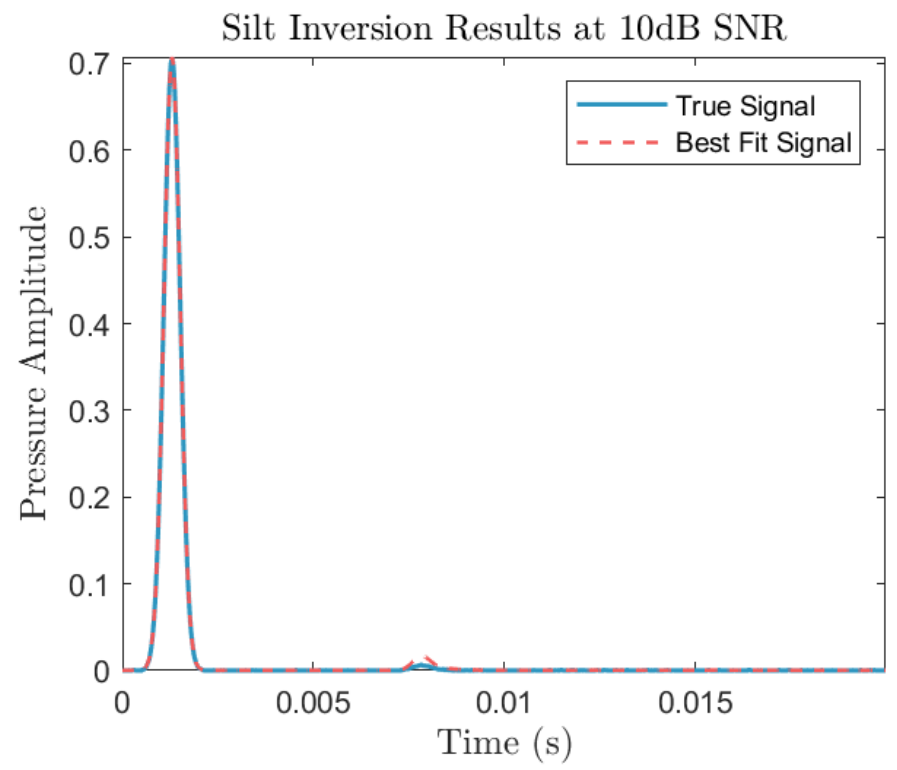

Figure 7.19: Real and estimated signal for silt surface at 10dB SNR

\subsection{Summary and Discussion of Results}

The results presented in the previous section demonstrate the classification method proposed in this thesis. The parameters $v, \rho, \gamma_{1}$ and $w_{1}$ were inverted for and the seabed type and roughness can be classified based on the estimated parameter values. The classifications are based on Table 6.1, which was presented in the APL High Frequency Handbook. The range of values for each seabed type are summarized in Table 7.10. The range of values for roughness type are defined in Table 7.11. These values will be used to gauge whether the estimated interface parameters correctly classify the seabed type and seabed roughness. 


\begin{tabular}{ccc}
\hline \multicolumn{3}{c}{ Parameter Ranges for Sediment Types } \\
\hline Sediment Type & $\begin{array}{c}\text { Density Ratio } \\
\rho\end{array}$ & $\begin{array}{c}\text { Sound Speed Ratio } \\
v\end{array}$ \\
Silt & $1.145-1.224$ & $0.9806-1.0364$ \\
Sand & $1.268-2.400$ & $1.0568-1.3370$ \\
Gravel & $2.5-2.6$ & $1.8-2.5$ \\
\hline
\end{tabular}

Table 7.10: Parameter Ranges for Sediment Type (adapted from (4))

\begin{tabular}{ccc}
\hline \multicolumn{3}{c}{ Parameter Ranges for Roughness Types } \\
\hline $\begin{array}{c}\text { Roughness Type } \\
\text { Spectral Exponent } \\
\gamma_{2}\end{array}$ & $\begin{array}{c}\text { Spectral Strength } \\
w_{2} \\
\left(\mathrm{~cm}^{4}\right)\end{array}$ \\
Smooth & $2.8-3.7$ & $0.000518-0.001119$ \\
Moderate & $2.8-3.7$ & $0.001544-0.008602$ \\
Rough & $2.8-3.7$ & $0.010573-0.20693$ \\
\hline
\end{tabular}

Table 7.11: Parameter Ranges for Roughness Type (adapted from (4)) 
The classification results for each sediment type are shown in table 7.12 .

\begin{tabular}{cccccc}
\hline \multicolumn{5}{c}{ Classification of Seabed Type Based on Estimated Parameters } \\
\hline Seabed Type & SNR & $\begin{array}{c}\text { Density Ratio } \\
\rho\end{array}$ & $\begin{array}{c}\text { Sound Speed } \\
\text { Ratio }\end{array}$ & Classification & $\begin{array}{c}\text { Successfully } \\
\text { Classified }\end{array}$ \\
Sand & $40 \mathrm{~dB}$ & 1.80 & 1.18 & Sand & Yes \\
Sand & 20dB & 1.70 & 1.26 & Sand & Yes \\
Sand & $10 \mathrm{~dB}$ & 1.56 & 1.54 & Undetermined & No \\
Gravel & $40 \mathrm{~dB}$ & 2.54 & 2.26 & Gravel & Yes \\
Gravel & 20dB & 2.6 & 2.5 & Gravel & Yes \\
Gravel & 10dB & 2.55 & 2.5 & Gravel & Yes \\
Silt & 40dB & 1.14 & 0.99 & Silt & Yes \\
Silt & 20dB & 1.18 & 0.98 & Silt & Yes \\
Silt & $10 \mathrm{~dB}$ & 1.09 & 1.03 & Silt & Yes \\
\hline
\end{tabular}

Table 7.12: Classification Results: Seabed Type 
The roughness classification results are shown in table 7.13. Note that $w_{1}$ and $\gamma_{1}$ are translated to $w_{2}$ and $\gamma_{2}$ using eq. 5.4 and eq. 5.3 .

\begin{tabular}{cccccc}
\hline \multicolumn{5}{c}{ Classification of Seabed Roughness Type Based on Estimated Parameters } \\
\hline Roughness Type & SNR & $\begin{array}{c}\text { Spectral } \\
\text { Exponent } \\
\gamma_{2}\end{array}$ & $\begin{array}{c}\text { Spectral Strength } \\
w_{2} \\
\left(\mathrm{~cm}^{4}\right)\end{array}$ & Classification & $\begin{array}{c}\text { Successfully } \\
\text { Classified }\end{array}$ \\
Moderate & $40 \mathrm{~dB}$ & 2.84 & 0.004 & Moderate & Yes \\
Moderate & $20 \mathrm{~dB}$ & 2.97 & 0.0044 & Moderate & Yes \\
Moderate & $10 \mathrm{~dB}$ & 3.3 & 0.0080 & Moderate & Yes \\
Rough & $40 \mathrm{~dB}$ & 3.12 & 0.0188 & Rough & Yes \\
Rough & $20 \mathrm{~dB}$ & 3.0 & 0.0158 & Rough & Yes \\
Rough & $10 \mathrm{~dB}$ & 3.3 & 0.0192 & Rough & Yes \\
Smooth & $40 \mathrm{~dB}$ & 2.84 & 0.00118 & Smooth & Yes \\
Smooth & $20 \mathrm{~dB}$ & 2.75 & 0.00146 & Moderate & No \\
Smooth & $10 \mathrm{~dB}$ & 3.3 & 0.000164 & Moderate & No \\
\hline
\end{tabular}

Table 7.13: Classification Results: Roughness Type

The results in Table 7.12 and Table 7.13 are promising, since the estimated values fall within the correct classification range. There is some discrepancy in the roughness classification for smooth surfaces and the estimated parameters. This could be caused by the true value of $w_{2}$ falling very close to the smooth- 
moderate boundary. Unfortunately the parameter $\gamma_{2}$ cannot be used to infer the roughness when the value of $w_{2}$ is near the boundary since it can take on the same range of values for any the roughness type.

The seabed type is correctly classified for all test cases except sand at low SNR. The returned value for sound speed does not fall within any specified range according to table 7.10 , which led to the classification being undetermined. If the value $\rho$ was enough to make a classification, however, sand would have been correctly classified. 


\section{Chapter 8}

\section{Conclusions and Future Work}

This research presented a seabed classification method based on measurements of normal incident acoustic reflections from a sidescan or single beam echosounder. New techniques that were utilized in seabed classification include:

- Inversion modeling was used for multiple parameter classification as opposed to prior work that searched for a single parameter.

- The forward model was validated against an exact and approximate solution at low frequencies. Most underwater acoustic research has utilized a higher frequency range, but lower frequencies have applications in seabed mapping, specifically with single beam echosounders.

- The inversion technique was not an exhaustive search over parameters; instead, differential evolution was applied to the parameter search space.

- Huber loss was used as the objective function to measure the difference in the data and modeled signals due to the robustness and tunability.

The results of the presented classification method are promising when compared with the APL Handbook definitions for bottom types; however, certain SNR values and seabed types were misclassified or undetermined. This leaves room for additional model and parameter tuning along with an exploration 
into the relationship between each parameter. The Huber loss function could be optimized by searching over different values of the $\delta$ parameter. Along with the step size and cross over probability in differential evolution.

Additionally, the inversion time could be extended to allow for more parameter combinations, especially at low SNR or the parameter search could be split into two parts. Initially, the sound speed and density could be found while keeping the other parameters fixed. After those parameters have been determined, set them as fixed and search over $w_{1}$ and $\gamma_{1}$. 


\section{Bibliography}

[1] P. C. Etter, Underwater acoustic modeling and simulation, vol. 3. London: Spon Press, 2003.

[2] D. Jackson and M. Richardson, High frequency ocean acoustics. New York: Wiley-InterScience, 2007.

[3] J. Anderson, D. Holliday, R. Kloser, D. Reid, Y. Simard, C. Brown, R. Chapman, R. Coggan, R. Kieser, W. Michaels, A. Orlowski, J. Preston, E. Simmonds, and A. Stepnowski, Acoustic seabed classification of marine physical and biological landscapes. 012007.

[4] S. Pinson, J. Cordioli, and L. Guillon, "Spherical wave reflection in layered media with rough interfaces: Three-dimensional modeling," The Journal of the Acoustical Society of America, vol. 140, pp. 1108-1115, 082016.

[5] M. Snellen, K. Siemes, and D. G. Simons, "Model-based sediment classification using single-beam echosounder signals," Jan 1970.

[6] F. Zheng, A. C. Zecchin, and A. R. Simpson, "Self-adaptive differential evolution algorithm applied to water distribution system optimization," Journal of Computing in Civil Engineering, vol. 27, no. 2, 2013.

[7] 2nd EAA International Symposium on Hydroacoustics, Inverse Problems in Underwater Acoustics, May 1999.

[8] D. Tang and D. Jackson, "A time-domain model for seafloor scattering," The Journal of the Acoustical Society of America, vol. 142, no. 5, 2017. 
[9] M. I. Taroudakis and G. Makrakis, Inverse problems in underwater acoustics. Springer, 2011.

[10] D. Chanchal, Characterization and Classification of Seafloor by Acoustic Method Using Model-Based and Model-Free Techniques. PhD thesis, Goa University, 2013.

[11] F. Jenson and ...[et al.], Computational ocean acoustics. New York: Springer Science, 2011.

[12] S. Rienstra and A. Hirschberg, An Introduction to Acoustics. 2004. https: //www.win.tue.nl/ sjoerdr/papers/boek.pdf.

[13] P. N. Duy, Physics Based Approach for Seafloor Classification. PhD thesis, Portland State University, 2017.

[14] X. Lurton, G. Lamarche, and ...[et al.], "Backscatter measurements by seafloormapping sonars guidelines and recommendations," tech. rep., Marine Geological and Biological Habitat Mapping, May 2015.

[15] T. Brückel, M. Angst, D. Richter, and R. Zorn, "Scattering theory: Born series," in Scattering Methods for Condensed Matter Research: Towards Novel Applications at Future Sources, ch. 2, pp. 11-15, 2012. http://juser.fz-juelich.de/record/20885/files/A2_Bluegel.pdf.

[16] T. Leighton, "Fundamentals of underwater acoustics and ultrasound," in Fahy, F.J., Walker, J.G., ed. Noise and vibration, vol. 1, ch. 7, pp. 373444, London: E\&F Spon, 1998. 
[17] I. Kraus, "Underwater acoustics and sonar signal processing." http: //homepages.hs-bremen.de/ krausd/iwss/UWA1.pdf.

[18] L. Bjørnø, Applied Underwater Acoustics. Saint Louis: Elsevier Science, 2017.

[19] N. Bleistein and J. Desanto, Ocean acoustics. Berlin: Springer-Verlag, 2011.

[20] A. Lyons, A. Anderson, and F. Dwan, "Acoustic scattering from the seafloor; modeling and data comparison," The Journal of the Acoustical Society of America, vol. 95, no. 2441, 1994.

[21] X. Lurton, An Introduction to Underwater Acoustics. SPRINGERVERLAG, 2016.

[22] L. Hamilton, "Acoustic seabed classification systems," tech. rep., Defence Science and Technology Organization Victoria (Australia) Aeronautical and Maritime Research Lab, 2001.

[23] P. Mulhearn, "Modelling acoustic backscatter from near-normal incidence echosounders - sensitivity analysis of the jackson model," Tech. Rep. DSTO-TN-0304, Maritime Operations Division Aeronautical and Maritime Research Laboratory, September 2000.

[24] W. U. S. A. P. Lab, "Apl-uw high-frequency ocean environmental acoustic models handbook," Tech. Rep. ADB199453, Office of Navel Research, October 1994. 
[25] D. McCammon and S. McDaniel, "Rough surface scattering and the kirchhoff approximation," in Merklinger H.M. (eds) Progress in Underwater Acoustics, Boston, MA: Springer, 1987.

[26] E. Thorsos and D. Jackson, "The validity of the perturbation approximation for rough surface scattering using a gaussian roughness spectrum," The Journal of the Acoustical Society of America, vol. 86, no. 261, 1988.

[27] D. Xiu and J. Shen, "An efficient spectral method for acoustic scattering from rough surfaces," Communications in Computational Physics, vol. 2, no. 1, pp. 54-72, 2006.

[28] L. Tsang, Scattering of electromagnetic waves. New York: WileyInterScience, 2001.

[29] C. Langston, "The effect of planar dipping structure on source and receiver responses for constant ray parameter," Bull. Seismol. Soc. Am., vol. 67, pp. 1029-1050, 1977.

[30] E. Thorsos, "The validity of the kirchhoff approximation for rough surface scattering using a gaussian roughness spectrum," The Journal of the Acoustical Society of America, vol. 83, no. 73, 1988.

[31] Y. Bard, Nonlinear Parameter Estimation. New York and London: Academic Press, 1974.

[32] R. Storn and K. Price, "Differential evolution -a simple and efficient 
heuristic for global optimization over continuous spaces," Journal of Global Optimization, no. 11, 1997.

[33] J. R. Collins, "Robust estimation of a location parameter in the presence of asymmetry.," The Annals of Statistics, vol. 4, no. 1, pp. 68-85, 1976.

[34] L. Wang, C. Zheng, W. Zhou, and W.-X. Zhou, A New Principle for Tuning-Free Huber Regression. 2019.

[35] H.-G. Beyer and H.-P. Schwefel, "Evolution strategies a comprehensive introduction," Natural Computing, vol. 1, 2002. 